\title{
Contribution à l'étude des écoulements permanents et variables dans les canaux
}

(FIN)

\section{Contribution to the study of permanent and non-permanent flows in channels \\ (CONCLUSION)}

\author{
par François SERRE
}

INGÉNEU CIVI DE L'ÉCOLE NATONALE DIS PONTS ET CUAUSSB́ES

\begin{abstract}
Importance et portée de l'hypothèse consistant à négliger la courbure des filets liquides. Etablissement des équations différentielles de l'écoulement permanent compte tenu des effets de courbure, d'une part en appliquant le théoréme des quantités de mouvement, d'autre part en appliquant le thérème de Bernoulli. L'exploitation de ces équations pour l'étude générale des courbes de remous.

Applications à la détermination des lignes de remous dans un certain nombre de cas classiques en régime permanent : écoulement fluvial e.t torrentiel, ressaut, ressaut ondulé (théorie du déferlement) - changements de pente et notion de section de contrôle - écoulements par vanne de fond - déversoirs à large. seuil (influence de la rugosité, ondulations de surface, résultats théoriques et expérimentaux). Etude du régime variable: établissement des équations aux dérivées partielles de l'écoulement. Le parti que l'on peul en tirer dans l'étude des intumescences, de la houle, du ressaut déferlant en régime variable.
\end{abstract}

\begin{abstract}
The importance and consequences of the hypothesis involving disregard of stream curvature. Development of differential equations for the permanent flow, taking into account the effects of curvature, by applying the momentum principle on the one hand, and the Bernoulli theorem on the other. Exploilation of these equations for the general study of backuater curves.

Applications to the determination of the backwater curve in a certain number of classical cases under permanent flow: tranquil and torrential flow, jump, undulated jump (breaking theory)-changes of bottom slopes and notion of control section-discharge under bottom gates-broad-crested weirs (influence of roughness, surface undalations, theoretical and experimental results).

Study of the non-permanent flow; formation of partial differential equalions of flow. The advantages which can be gained in the study of swell, waves, breaking jump nuder variable now.
\end{abstract}

\section{IV. - DETERMINATION DES LIGNES DE REMOUS POSITION DU PROBLÈME}

Considérons un canal de section rectangulaire de grande largeur, constitué de difírentes portions de mème largeur mais de pentes différentes. A l'amont et à l'aval, le canal peut soit s'étendre à l'infini, soil communiquer avec un

(*) CA. la Houille Blanche, ${ }^{*} 3,1953$, p. 374 . réservoir dont on connait la cole du plan d'eau au-dessus du radier.

Dans une portion quelconque de pente $i$, la charge $\mathrm{H}$ et la hauteur d'eau $y$ au-dessus du radier satisfont au systeme différentiel (9) correspondant à la valeur $i$ de la pente. Pour un débit $Q$ donné, la détermination de la ligne d'eau nécessite la connaissance de trois conditions 
particulières, par exemple les conditions de CaUCHY au point d'abscisse $x=x_{0}$ :

$$
x=x_{0}\left\lceil y\left(x_{0}\right), \mathrm{H}\left(x_{0}\right),\left(\frac{d y}{d x}\right)_{x=y_{0}}\right\rceil
$$

En fait, le problème sera déterminé par les conditions particulières aux extrémités de la portion envisagée. Avant d'examiner les conditions aux limites dans le cas général, nous conlmencerons par étudier quelques cas particuliers.

\section{1. - Canal de pente uniforme $i$, s'étendant à l'infini à l'amont et débouchant à l'aval dans un réservoir $B$ dont on connaît la cote $y_{1}$ du plan d'eau au-dessus du radier.}

Nous prendrons pour origine des coordonnées $O$ l'extrémité aval du canal.

Le canal s'étendant à l'infini à l'amont, le régime uniforme doit être réalisé lorsque $x$ augmente indéfiniment par valeurs négatives. Les solutions du système (9) qui satisfont à cette condition sont, nous l'avons vu, représentées dans le plan $\mathrm{HO} y$ par une courbe $(\mathrm{C})$ unique $\left(\mathrm{C}_{0}\right)$ d'équation $\mathrm{H}=\mathrm{H}_{0}(y)$

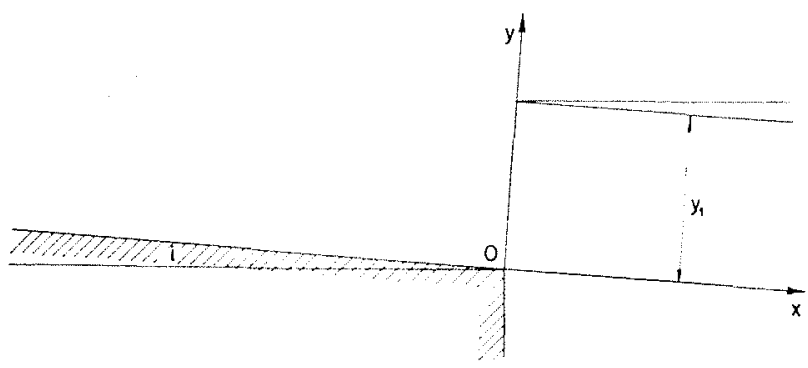

Fic. 14

La deuxième équation de (9) montre que les lignes de remous correspondantes sont les solutions de l'équation différentielle du premier ordre à variables séparées :

$$
\frac{d \mathrm{H}_{11}}{d y} \frac{d y}{d x}=i \quad \frac{\mathrm{Q}^{2}}{c^{2} l^{2} y y^{3}}
$$

La ligne d'eau cherchée est la solution particulière qui satisfait aux conditions de CAUCHY $\left(x=0 \quad y=y_{1}\right)$.

Notons que les courbes intégrales de (16) se déduisent toutes de l'une d'entre elles par une translation parallèle à $O x$. Le tracé d'une quelconque d'entre elles se déduit de la courbe $\left(\mathrm{C}_{0}\right)$ du plan $\mathrm{HO} y$.

Deux cas sont à envisager suivant que le régime est fluvial ou torrentiel dans le canal.

\section{a) Régime fluvial.}

La hauteur normale $y_{0}$ est supérieure à la hauteur critique $y_{6}$. La courbe $\left(C_{0}\right)$ passe, nous l'avons vu, par le point $\mathrm{P}$ d'intersection de la courbe $(\mathrm{H})$ d'équation $\mathrm{H}=y+\mathrm{Q}^{2} /\left(2 g l^{2} y^{2}\right)$ et de la parallèle à $\mathrm{OH}$ d'abscisse $y=y_{0}$, sa tangente en ce point ayant pour valeur :

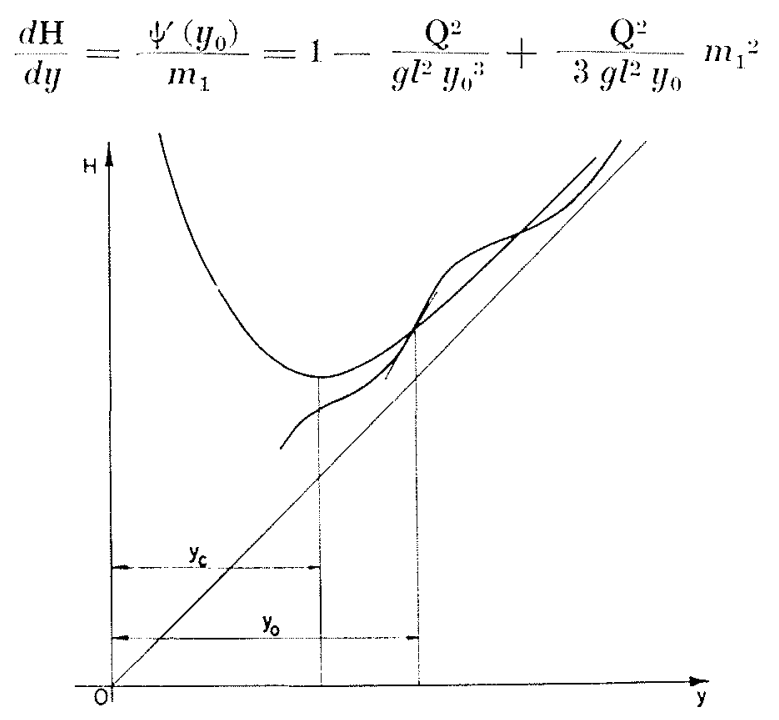

FIG. 15

$m_{1}$ élant la racine réelle positive de l'équation :

$$
\frac{\mathrm{Q}^{2}}{3 g l^{2} y_{0}} m_{1}^{*}+\left(1-\frac{Q^{2}}{g l^{2} y_{0}^{3}}\right) m_{1}=\psi^{\prime}\left(y_{0}\right)
$$

et :

$$
\psi(y)=i-\frac{Q^{2}}{c^{2}(y) l^{2} y^{3}}
$$

$\left(\mathrm{C}_{0}\right)$ est, comme toutes les courbes (C), asymptote a $(\mathrm{H})$ et elle recoupe une infinité de fois la courbe intégrale particulière $\left(\mathrm{C}_{1}\right)$ du système (10) d'équation $\mathrm{H}=\mathrm{H}_{1}(y)$.

Le tracé de $\left(C_{0}\right)$ s'en déduit. Sur cette courbe, $y$ et $\mathrm{H}$ sont définis paramétriquement en fonction de $x$, le point $\mathrm{P}$ étant atteint, nous l'avons vu, pour $x=-\infty$ et le point à l'infini pour $x=+\infty$. Nous avons également démontré que toutes les courbes de remous possédaient une asymptote horizontale.

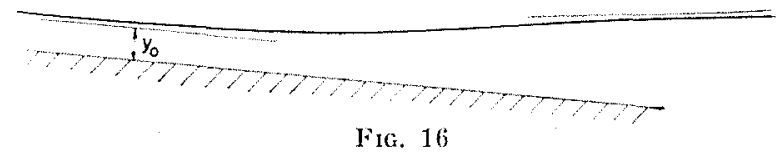


La branche de $\left(\mathrm{C}_{0}\right)$, située à gauche de $\mathrm{P}$, est située tout entière au-dessous de $(\mathbf{H})$. L'étude du point singulier $y=0$ du système (9) montrerait que lorsque $y$ tend vers $0, H$ tend vers $-\infty$. Cette branche correspond au cas où la hauteur d'eau dans le réservoir aval $B$ est inférieure à la hauteur du régime uniforme $y_{0}$.

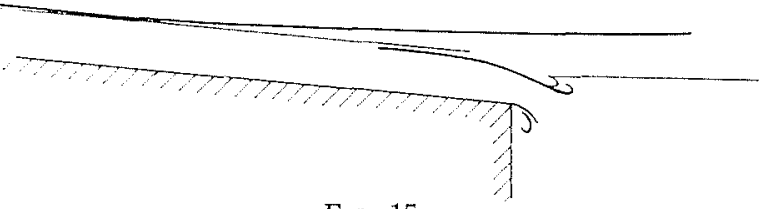

FIG. 17

\section{b) Régime TORRENTIEL}

La hauteur normale $y_{0}$ est inférieure à la hauteur critique $y_{c}$.

Dans ce cas, la courbe $\left(\boldsymbol{C}_{0}\right)$ possède un certain nombre de points où la tangente est parallèle a $\mathrm{OH}$.

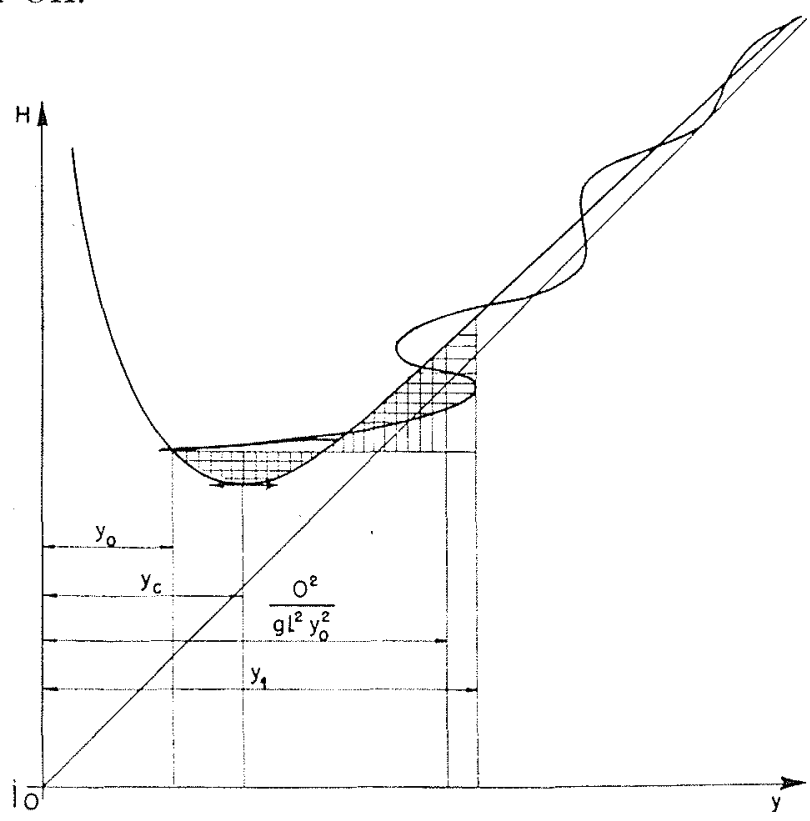

FIG. 18

Si $y_{1}$ désigne l'abscisse du premier de ces points (fig. 18), la première équation de (9) mise sous la forme :

$$
\frac{\mathrm{Q}^{2}}{6 g l^{2} y} \frac{d}{d y}\left[\frac{1}{y}\left(\frac{d y}{d x}\right)^{2}\right]=\mathrm{H}-y-\frac{\mathrm{Q}^{2}}{2 g l^{2} y^{2}}
$$

conduit, en tenant compte de $:\left(\frac{d y}{d x}\right)_{y=y_{0}}=0$

à la relation :

$$
\int_{y_{0}}^{y_{1}}\left[\mathrm{H}(y)-y-\frac{\mathrm{Q}^{2}}{2 g^{2} y^{2}}\right] d y=0
$$

dont l'interprétation géométrique est évidente (aires hachurées horizontalement égales).

On obtient un renseignement plus précis sur Ia valeur de $y_{1}$ et sur la forme de la courbe de remous en négligeant la variation de $H$ lorsque $y$ croît de $y_{0}$ à $y_{1}$, cette variation étant liée $\grave{a} x$ par la deuxième équation de (9):

$$
\frac{d \mathrm{H}}{d x}=i-\frac{\mathrm{Q}^{2}}{c^{2} l^{2} y^{3}}
$$

Intégrons l'équation :

$$
\frac{\mathrm{Q}^{2}}{6 g l^{2}} \frac{d}{d y}\left[\frac{1}{y}\left(\frac{d y}{d x}\right)^{2}\right\rfloor=\mathrm{H}-y-\frac{\mathrm{Q}^{2}}{2 g l^{2} y^{2}}
$$

en admettant que $H$ garde sa valeur pour :

$$
y=y_{0} \quad \mathrm{H}=\mathrm{H}_{0}=y_{0}+\frac{\mathrm{Q}^{2}}{2 g^{2} y_{0}{ }^{2}}
$$

il vient :

$$
\frac{\mathrm{Q}^{2}}{6 g^{2} y}\left(\frac{d y}{d x}\right)^{2}=\mathrm{H}_{0} y-\frac{y^{2}}{2}+\frac{\mathrm{Q}^{2}}{2 g l^{2} y}+\mathrm{C}
$$

Ia constante arbitraire étant déterminée par la condition $\left(\frac{d y}{d x}\right)_{y=y_{0}}=0$.

d'où :

$$
\mathrm{C}=-\frac{y_{0}^{2}}{2}-\frac{\mathrm{Q}^{2}}{g l^{2} y_{0}}
$$

L'équation précédente s'écrit alors :

$$
\frac{\mathrm{Q}^{2}}{3 g l^{2}}\left(\frac{d y}{d x}\right)^{2}=\left(y \cdot-y_{0}\right)^{2}\left[\frac{\mathrm{Q}^{2}}{g^{2} y_{0}^{2}}-y\right]
$$

Les courbes intégrales de cette équation du premier ordre se déduisent toutes de l'une d'entre elles (S) par une translation parallèle à l'axe Ox. La forme de (S) est précisée sur la figure (19).

$y$ est compris entre $y_{0}$ et $\mathrm{Q}^{2} / g 7^{2} y_{0}{ }^{2}$. Le deuxième membre de (17) devant être positif, il n'existe une courbe (S) réelle asymptote à $y=y_{0}$ que si :

$$
y_{0}<\frac{\mathrm{Q}^{2}}{g l^{2} y_{0}^{2}}, \text { c'est-à-dire } y_{0}<\sqrt[3]{\frac{\overline{\mathrm{Q}^{2}}}{g l^{2}}}=y_{c}
$$

qui est précisément la condition du régime torrentiel.

Dans le plan $\mathrm{HOy}$, (S) est représentée par le segment de la droite :

$$
\mathrm{H}=\mathrm{H}_{0}=y_{0}+\frac{\mathrm{Q}^{2}}{2 g l^{2} y_{0}^{2}}
$$

compris entre les points d'abscisse:

$$
y=y_{0} \quad y=\frac{\mathrm{Q}^{2}}{g l^{2} y_{0}{ }^{2}}
$$


D'après une remarque déjà faite, les aires hachurées verticalement sur la figure 18 sont égales. égales.

L'examen de cette figure montre immédiatement que lordonnée $y_{1}$ du premier point de la courbe de remous, où la tangente est parallèle à $O x$, est supérieure à $\mathrm{Q}^{2} / g l^{2} y_{0}{ }^{2}$.

Par ailleurs, les courbes de remous (R) et $(S)$ satisfont respectirement aux deux équations :

(R) $\frac{\mathrm{Q}^{2}}{6 g l^{2} y}\left(\frac{d y}{d x}\right)^{2}=\int_{y_{0}}^{y}\left[\mathrm{H}(u)-u-\frac{\mathrm{Q}^{2}}{2 g l^{2} u^{2}}\right] d u$

(S) $\frac{\mathrm{Q}^{2}}{6} \frac{d y}{l^{2} y}\left(\frac{d y}{d x}\right)^{2}=\int_{y_{0}}^{y}\left[\mathrm{H}_{0}-u-\frac{Q^{2}}{2 g^{l^{2}} u^{2}}\right] d u$

comme pour $y>y_{0} \mathrm{H}(u)>\mathrm{H}_{0}$, on en déduit qu'en deux points de même ordonnée la pente de $(R)$ est supérieure à celle de (S) [confers fig. 19].

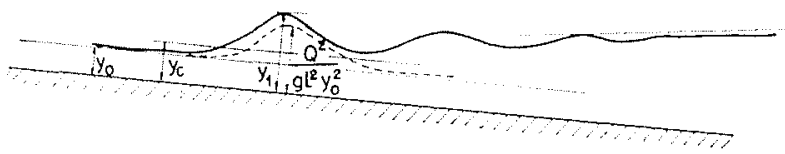

FIG. 19

De ces considérations on déduit aisément le tracé de la courbe $\left(\mathrm{C}_{0}\right)$ et de la ligne de remous (R). Nos équations traduisent ainsi un fait expérimental classique concernant le passage de l'écoulement torrentiel à l'écoulement fluvial et connu sous le nom de ressaut ondulé (undulated jump).

Ce phénomène est caractérisé par une remontée relativement progressive de la surface libre suivie de vagues à emplacement fixe dont la hauteur va en décroissant.

Il est observé lorsque la hauteur normale $y_{0}$, tout en étant inférieure à la hauteur critique $y_{c}$, ne lui est pas trop inférieure.

Pour une valeur suffisamment faible du rapport $y_{0} / y_{0}$, la première onde devient instable et sa partie supérieure commence à déferler

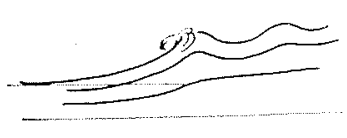

Fig. 20

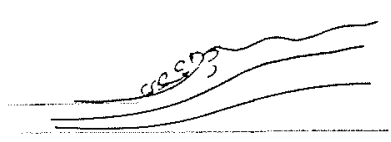

FIG. 21 (fig. 20), ce qui tend à amortir les ondulations suivantes. Pour $y_{0} / y_{c}$ assez petit, ces dernières finissent par disparaître entièrement et le déferlement, accompagné de bouillonnements intenses, vient recouvrir la partie la plus basse de la ligne d'eau, le phénomène prenant l'allure de la fi- gure 21. C'est le ressaut déferlant (direct jump). Les particules d'eau engagées dans le tourbillon à axe horizontal qui constitue la partie déferlante sont animées d'un mouvement de rotation et ne participent pas au mouvement de translation du fluide.

Cette discontinuité de la surface libre et la perte de charge singulière qu'elle provoque rendent dans ce cas les équations (9) inapplicables.

Mais la localisation du ressaut permet alors d'appliquer le théorème des quantités de mouvement entre les sections (1) et (2) en négligeant l'inclinaison du radier du canal et les forces de frottement entre les deux sections. Ceci nous conduit à écrire que la fonction $M(x)$ a la même valeur dans les sections (1) et (2), c'est-à-dire à la thérie classique du ressaut, en admettant pour M la valeur de première approximation :

$$
\mathrm{M}=\frac{y^{2}}{2}+\frac{\mathrm{Q}^{2}}{g l y}
$$

Cette approximation est rigoureuse dans (1)

$$
\text { ò } \frac{d y}{d x}=\frac{d^{2} y}{d x^{2}}=0 \text {. }
$$

\section{Théorie du Défertement.}

Considérons une particule fluide située sur la surface libre. Elle est en équilibre sur sa trajectoire sous l'action des forces extérieures (pesanteur), des forces d'inertie et des forces de liaison qui constituent la poussée du milieu environnant. Pour que la particule ne tende pas à quitter la surface libre, il faut que la composante normale à la trajectoire de cette poussée soit dirigée vers l'extérieur du fluide.

La valeur de cette composante sur la normale extérieure :

$$
\mathrm{R}=m\left(g \cos \alpha+\frac{\mathrm{V}^{2}}{?}\right)
$$

$\alpha$ étant l'angle de la normale avec la verticale :

$$
\cos \alpha=\left(1+y^{\prime 2}\right)^{-1 / 2}
$$

p rayon de courbure de la trajectoire:

$$
\uparrow=\frac{\left(1+y^{\prime 2}\right)^{3 / 2}}{y^{\prime \prime}}
$$

$\checkmark$ vitesse de la particule:

$$
V^{2}=u^{2}+w^{2}=u^{2}\left(1+y^{\prime 2}\right)=\frac{Q^{2}}{l^{2} y^{2}}\left(1+y^{\prime 2}\right)
$$

d'où :

$$
\mathrm{R}=m g\left(1+y^{\prime 2}\right)^{-1 / 2}\left[1+\frac{\mathrm{Q}^{2} y^{\prime \prime}}{g l^{2} y^{2}}\right\rceil
$$


on en déduit la condition de non déferlement:

$$
\frac{Q^{2}}{g l^{2} y^{2}} \frac{d^{2} y}{d x^{2}}+1>0
$$

En premiere approximation, examinons comment se traduit cette condition, lorsqu'on confond la première onde avec la courbe (S), définie par l'équation différentielle :

$$
\frac{\mathrm{Q}^{2}}{3 g l^{2}}\left(\frac{d l}{d x}\right)^{2}=\left(y-y_{0}\right)^{2}\left(\frac{\mathrm{Q}^{2}}{g l^{2} y_{0}^{2}}-y\right)(17)
$$

il vient :

$\frac{\mathrm{Q}^{2}}{g^{2} y^{2}} \frac{d^{2} y}{d x^{2}}=\frac{3}{2} \frac{\left(y-y_{0}\right)\left[2 \frac{Q^{2}}{l^{2}}+g y_{0}^{3}-3 g y_{0}^{2} y\right]}{g y_{0}^{2} y}$

posons :

$$
\lambda=\frac{Q^{2}}{g^{l^{2}} y_{0}^{3}} \quad u=\frac{y}{y_{0}}
$$

En fonction de ces variables sans dimensions :

$$
\begin{aligned}
& \frac{\mathrm{Q}^{2}}{g^{l^{2}} y^{2}} \frac{d^{2} y}{d x^{2}} \text { a pour expression : } \\
& \frac{\mathrm{Q}^{2}}{g^{2} y^{2}} \frac{d^{2} y}{d x^{2}}==\frac{3}{2} \frac{(u-1)(2 \lambda+1-3 u)}{u^{2}}
\end{aligned}
$$

dont nous étudierons la variation lorsque $y$ croil de $y_{0}$ à $\frac{Q^{2}}{g l^{2} y_{0}{ }^{2}}$, c'est-à-dire lorsque $u$ crồt de 1 à $\lambda$
La valeur minima de cette fonction dans cet intervalle s'obtient pour $u=\lambda$, c'est-a-dire :

$$
y=\frac{Q^{2}}{g^{2} y_{0}^{2}}
$$

el son expression est :

$$
-\frac{3}{2} \frac{(i-1)^{2}}{2 ! 2}
$$

Le déferlement commence donc à apparaître dans la partie supérieure de la première onde.

La condition de non-déferlement s'écril :

soit :

$$
-\frac{3}{2} \frac{(\lambda-1)^{2}}{\lambda^{2}}+1>0
$$

comme :

$$
\frac{1}{3}>1-\sqrt{\frac{2}{3}}
$$

$$
\frac{1}{\lambda}=\frac{g l^{2} y_{0}^{2}}{\mathrm{Q}^{2}}=\left(\frac{y_{0}}{y_{0}}\right)^{2}
$$

la condition précédente s'écrit :

$$
\frac{y_{11}}{y_{1}}>\sqrt[3]{1-\sqrt{\frac{2}{3}}}=0,57
$$

En pratique, le deferlement apparait avant que $y_{0} / y_{c}$ soit inférieur à 0,57 , l'influence du frottement de lair sur la surface libre jouant à cet égard un rôle particulier. Un facteur important d'instabilité est également constitué par le fait que l'écoulement n'est permanent qu'en moyenne, la surface libre oscillant autour d'une position moyenne. Les efforts dynamiques qui en résultent sur la particule d'eau contribuent à son instabilité.

\section{2. - Canal constitué de deux portions de pente différentes.}

Le canal s'étend à l'infini à l'amont, à l'aval il peut soit s'étendre à l'infini, soit déboucher dans un réservoir (B) où le plan d'eau est fixe.

Nous supposerons la pente faible dans la portion amont et forte dans la portion aval.

Soient $i_{1}$ et $i_{2}$ les pentes respectives des por-

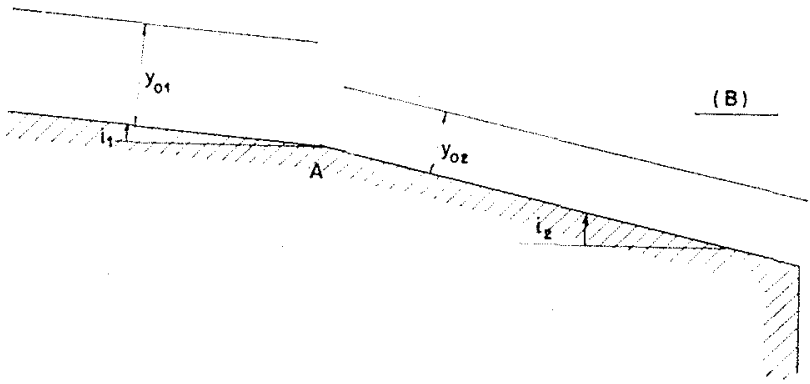

Fig. 22 tions amont et aval; désignons par $y_{01}$ et $y_{102}$ les hauteurs normales correspondantes :

$$
y_{02}<y_{0}<y_{01} \quad i_{2}>i_{1}
$$

Dans chaque portion de canal, les lignes de remous $\left(R_{1}\right)$ et $\left(R_{0}\right)$ vérifient le système $(9)$ respectivement pour les valeurs $i_{1}$ ef $i_{2}$ du paramètre $i$.

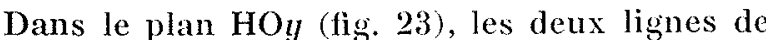
remous sont représentées respectivement par deux courbes $\left(\mathrm{C}_{1}\right)$ et $\left(\mathrm{C}_{2}\right)$, solutions du système (10):

$$
\left\{\begin{array}{l}
\frac{\mathrm{Q}^{2}}{6 g l^{2}} \frac{d}{d y}\left(\frac{z^{2}}{y}\right)=\mathrm{H}-y-\frac{\mathrm{Q}^{2}}{2 g l^{2} y^{2}} \\
z \frac{d \mathrm{H}}{d y}=i-\frac{\mathrm{Q}^{2}}{c^{2} l^{2} y^{3}}
\end{array}\right.
$$


Soit $h$ la hauteur d'eau réalisée dans la seclion A séparant les deux portions du canal. Pour $y=h$, les deux systèmes de solution de (10):

$z=z_{1}(y) \quad \mathrm{H}=\mathrm{H}_{1}(y), \quad z=--z_{2}(y) \quad \mathrm{H}=\mathrm{H}_{2}(y)$

doivent vérifier les conditions :

$H_{1}(h)=H_{2}(h)$, (pas de variation de la charge)

$$
\left(\frac{d y}{d x}\right)_{2}-\left(\frac{d y}{d x}\right)_{1}=z_{2}(h)-z_{1}(h)=i_{2}-i_{1}
$$

qui expriment la continuité de la surface libre $\left[\left(\mathrm{R}_{1}\right)\right.$ et $\left(\mathrm{R}_{2}\right)$ tangentes pour $\left.x=0 \quad y=h\right]$.

CONDITION A L'EXTRÉmITÉ AMONT.

Le canal s'éloignant à l'infini à l'amont, la hauteur normale doit ètre réalisée loin à l'amont de $A$, ce qui exige que la courbe $\left(C_{1}\right)$ soit la courbe $\left(\mathrm{C}_{0}\right)$ correspondant à la valeur $i_{1}$ de $i$. Nous désignerons cette courbe par $\left(\mathrm{C}_{01}\right)$. Elle est tracée sur la figure 23.

A cette courbe $\left(\mathrm{C}_{01}\right)$ correspond, ainsi qu'on l'a vu, une infinité de lignes d'eau se déduisant les unes des autres par une translation parallèle à l'axe $O x$, la ligne $\left(\mathrm{R}_{1}\right)$ cherchéc est celle qui correspond aux conditions en $\mathrm{A}\left[\begin{array}{ll}x=0 & y=h\end{array}\right]$.

Quant à la courbe $\left(\mathrm{C}_{2}\right)$, c'est la courbe intégrale du système:

$$
\left\{\begin{array}{l}
\frac{\mathrm{Q}^{2}}{6 g^{2}}-\frac{d}{d y}\left(\frac{z^{2}}{y}\right)=\mathrm{H}-y-\frac{\mathrm{Q}^{2}}{2 g l^{2} y^{2}} \\
z \frac{d \mathrm{H}}{d y}=i_{2}-\frac{\mathrm{Q}^{2}}{c^{2} l^{2} y^{3}}
\end{array}\right.
$$

qui satisfait aux conditions de Cauchy.

$$
\begin{aligned}
& y=h \quad \mathrm{H}(h)=\mathrm{H}_{01}(h) \\
& z(h)=z_{01}(h)+i_{2.2}-i_{1}
\end{aligned}
$$

$\mathrm{H}=\mathrm{H}_{01}(y)$ désignant l'équation de la courbe $\left(\mathrm{C}_{01}\right)$ (fig. 23) et $z_{01}(y)$ étant donné par l'équation :

$$
z_{01}(y) \frac{d \mathbf{H}_{n 1}}{d y}=i_{1}-\frac{\mathrm{Q}^{2}}{c^{2} l^{2} y^{3}}
$$

A cette courbe $\left(C_{.}\right)$correspondent une infinité de lignes de remous se déduisant les unes des autres par une translation parallèle à l'axe $O x$, la ligne $\left(\mathbf{R}_{2}\right)$ cherchée étant celle qui correspond aux conditions en $\mathrm{A}[x=0, y=h]$.

En conclusion, à chaque hauteur d'eau $h$ dans la section $A$ correspondent deux lignes de remous $\left(R_{1}\right)$ et $\left(R_{2}\right)$ à l'amont et à l'aval de $A$ qui satisfont aux conditions aux limites dans la section $\mathrm{A}$ et à l'infini amont.
La hauteur d'eau réelle dans la section A sera déterminée par les conditions aval. Pour étudier les diverses formes de la ligne d'eau lorsqu'on fait varier les conditions aval, nous prendrons le problème à l'envers, en faisant varier la hauteur d'eau dans la section $A$.

L'allure des courbes de remous se déduit de l'examen des courbes $\left(\mathrm{C}_{2}\right)$ tracées dans le plan $\mathrm{HO} y$, pour diverses valeurs de $h$ (fig. 23).

Supposons par exemple que $h=y_{01}$ (hauteur du régime uniforme dans la portion amont). La ligne de remous $\left(R_{1}\right)$ à l'amont de $A$ est la droite $y=y_{01}$ correspondant au régime normal. Dans

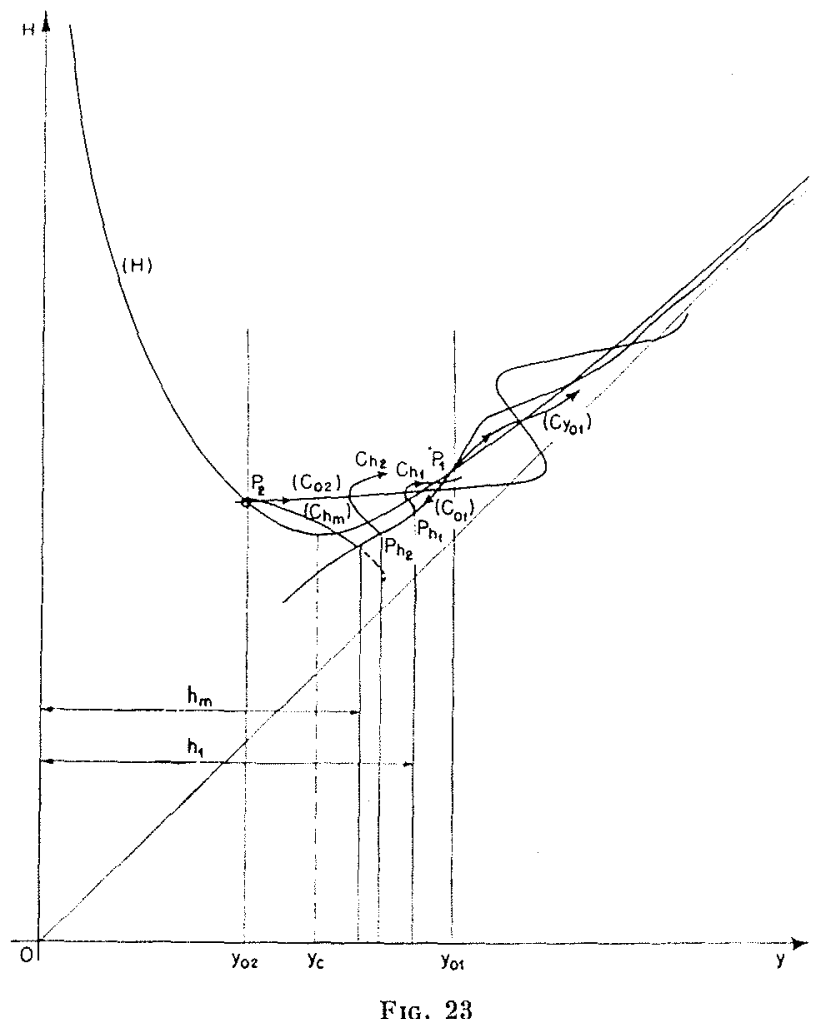

le plan $\mathrm{HOy}$, cette droite est représentée par le point $\mathrm{P}_{1}$.

La ligne de remous $\left(R_{2}\right)$ à l'aval de $A$ est représentée dans le plan ( $\mathrm{HOy}$ ) par une courbe $\left(\mathrm{C} y_{01}\right)$ intégrale des équations $(10)_{2}$ passant par le point $\mathrm{P}_{1}$. La pente de $\left(\mathrm{C} y_{01}\right)$ en $\mathrm{P}_{1}$ a pour valeur :

$$
\left(\frac{d \mathrm{H}}{d y}\right)_{y=y_{01}}=\frac{i_{2}-\frac{\mathrm{Q}^{2}}{c^{2} l^{2} y_{01}{ }^{3}}}{z\left(y_{01}\right)}
$$

or :

$$
\begin{aligned}
z\left(y_{01}\right) & =z_{01}\left(y_{01}\right)+i_{2}-i_{1} \\
z_{01}\left(y_{01}\right) & =\left(\frac{d y}{d x}\right)_{1, y=y_{01}}=0
\end{aligned}
$$


comme d'après la définition du niveau normal :

$$
i_{1}=\frac{\mathrm{Q}^{2}}{c^{2} l^{2} y_{01}^{3}}
$$

on voit que:

$$
\left(\frac{d \mathrm{H}}{d y}\right)_{y=y_{01}}=1
$$

La courbe $\left(\mathrm{C} y_{01}\right)$ correspondante est tracée sur la figure 23, elle est très voisine de $(\mathbf{H})$. La courbe de remous $\left(R_{2}\right)$ s'en déduit immédiatement.

Si la hauteur $h$ dans la section A est supérieure à $y_{01}$, $\left(\mathrm{R}_{1}\right)$ est représentée dans le plan $(\mathrm{HOy})$ par un arc $\left(\mathrm{P}_{1} \mathrm{P}_{h}\right)$ de la courbe $\left(\mathrm{C}_{01}\right)$, situé à droite de $P_{1}$, et $R_{2}$ est représentée dans le même plan par une courbe $\left(\mathrm{C}_{h}\right)$ passant par le point $P_{h}$ et admettant en ce point une tangente déterminée; la courbe $\left(\mathrm{C}_{k}\right)$ est encore très voisine de $(\mathrm{H})$.

Lorsque la hauteur $h$ dans la section A est inférieure à $y_{01}$, le point $\mathrm{P}_{h}$ est à gauche de $\mathrm{P}_{1}$, la pente de la tangente à $\left(C_{h}\right)$ au point $P_{h}$ augmente. Cette pente a en effet pour valeur :

$$
\left(\frac{d \mathrm{H}}{d y}\right)_{y=n}=\frac{i_{2}-\frac{\mathrm{Q}^{2}}{c^{2} l^{2} h^{3}}}{z(h)}
$$

où :

$$
z(h)=z_{01}(h)+i_{2}-i_{1}
$$

or :

$$
z_{01}(h)=\left(\frac{d ! y}{d x}\right)_{1_{y=h}}
$$

est la pente par rapport au radier amont de la ligne de remous $\left(R_{1}\right)$, pente négative pour $h<y_{01}$. $z(h)$ est donc une fonction décroissante de $h$ et elle s'annule pour une certaine valeur $h_{1}$. On a alors :

$$
\left(\frac{d \mathbf{H}}{d y}\right)_{y=n_{1}}=\infty
$$

La courbe $\left(C_{n 1}\right)$ est tracée sur la figure 23; elle possède, outre $P_{h 1}$, un certain nombre de points où la tangente est parallèle à $\mathrm{OH}$. Comme toutes les courbes (C), elle est d'ailleurs asymptote à $(\mathrm{H})$. Lorsque $y$ augmente indéfiniment, la courbe de remous correspondante présente un certain nombre d'ondulations dont l'amplitude va en s'amortissant lorsque la profondeur d'eau augmente, la ligne d'eau possédant, comme nous l'avons montré, une asymptote horizontale.

L'amplitude de la première ondulation va en augmentant lorsque la hauteur d'eau dans la section $\mathrm{A}$ devient inférieure à $h_{1}$. La pente de la tangente à $\left(\mathrm{C}_{n}\right)$ en $\mathrm{P}_{h}$ devient alor's négative; lo point le plus bas de la ligne d'eau à l'aval de $A$ se rapproche du niveau normal. En particulier, pour une certaine valeur $h_{m}$ de $h$, la courbe $\left(\mathbf{C}_{l m}\right)$ qui passe par $\mathrm{P}_{h m}$ passe par le point $P_{2}$ de coordonnées :

$$
y=y_{02}, \quad \mathrm{H}=\mathrm{H}_{02}=y_{02}+\mathrm{Q}^{2} /\left(2 g l^{2} y_{02}{ }^{2}\right) \text {, }
$$

ce point étant atteint lorsque $x$ augmente indèfiniment par valeurs positives.

Nous avons vu en effet qu'il existe une simple infinité de courbes (C) qui satisfont à cette condition. Ces courbes sont donc les courbes intégrales d'une équation du premier ordre :

$$
f\left(y, \mathrm{H}, \frac{d \mathrm{H}}{d y}\right)=0
$$

Comme au point $\mathrm{P}_{h}, \mathrm{H}$ et $d \mathrm{H} / d y$ sont des fonctions bien déterminées de $h$, l'équation précódente est une équation en $h$ qui permet do déterminer $h_{m}$.

La position du point $P_{l m}$ sur la figure 23 a été déterminée par continuité, en utilisant la propriété démontrée plus haut concernant l'aire comprise entre une courbe $(\mathrm{C})$ et la courbe $(\mathrm{H})$

La ligne de remous $\left(R_{2}\right)$ correspondante est asymptote à la droite $y=y_{02}$ du régime uniforme.

Il est facile de se rendre compte que la hauteur d'eau dans la section A ne peut ètre inférieurc $\dot{a} h_{m}$, car la courbe $\left(\mathrm{C}_{h}\right)$ correspondante (voir figure 23) serait située entièrement à gauche de $\mathrm{P}_{l}$, la hauteur d'eau à l'aval de A décroîtrait sans cesse of la courbe de remous correspondante ne pourail satisfaire aux conditions aval.

Le tracé des diverses courbes de remous col-

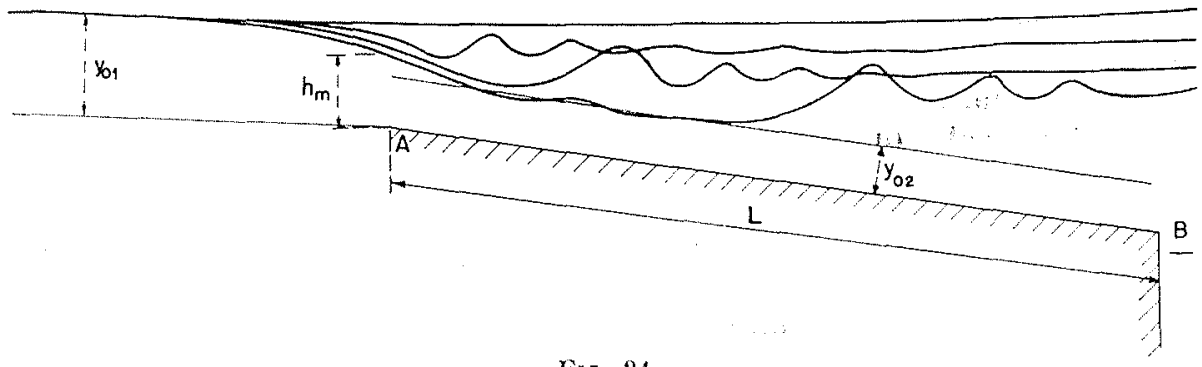

FIG. 24 
respondant aux diverses hauteurs d'eau dans la section A est figuré sur la figure 24. Ces lignes d'eau sont obtenues lorcu'on fait varier les conditions aval, par exemple la hauteur d'eau dans un réservoir $B$ situé à une distance $L$ à l'aval de $A$.

Lorsque le niveau de l'eau dans B diminue, la hauteur d'eau dans la section A décroît. Au-dessous d'une certaine cote $h_{\mathfrak{P}}$ du plan d'eau dans le réservoir $B$, il est impossible d'obtenir une ligne de remous $\left(R_{2}\right)$ satisfaisant aux conditions limites en A et B, la hauteur d'eau dans la section A devient indépendante des conditions aval, tout se passant comme si le canal de pente $i_{2}$ s'éloignait indéfiniment à l'aval. La ligne de remous est asymptote au régime uniforme $y=y_{02}$, la hauteur d'eau en A étant précisément $h_{n: 2}$ Dans le plan $\mathrm{HO} y$ (fig. 23), la courbe figurative est $\left(\mathrm{C}_{l m m}\right)$.

A l'amont de $B$, tout se passe également comme si le canal de pente $i_{2}$ s'éloignait indéfiniment à l'amont. La ligne de remous se détermine alors d'après les considérations du paragraphe (1), la courbe figurative dans le plan $\mathrm{HO}$ y etant $\left(\mathrm{C}_{02}\right)$.

On retrouve ainsi la notion classique de « section de contròle $»$.

Le problème se résoudrait de la même facon si le canal comportait un plus grand nombre de portions de pente différentes. Nous allons maintenant préciser les conditions aux limites à l'extrémité amont.

\section{3. - Canal communiquant à l'amont avec un réservoir $\mathbf{A}$, l'admission de l'eau étant réglée par une vanne.}

Désignons par $\mathrm{H}$ la cote du plan d'eau dans le réservoir $\mathrm{A}$, au-dessus du radier du canal; $a$, l'ouverture de la vanne.

Nous supposerons que le canal de pente $i$ s'éloigne à l'infini à l'aval.

Le problème se pose de la facon suivante :

Pour des valeurs données de $a$ et de $\mathrm{H}$, quel est le débit $Q$ dans le canal et la forme de la ligne d'eau?

Les conditions aux limites à l'extrémité amont seront obtenues en appliquant le théoreme de Bernoulli et le théorème des quantités de mouvement entre la section (1) située au droit de la vanne et la section (2) située dans la portion de canal à l'amont de la vanne, suffisamment loin de celle-ci pour que la répartition des vitesses puisse y être considérée conme uniforme.

Nous négligerons également la pente et la courbure de la ligne d'eau dans la section (2)

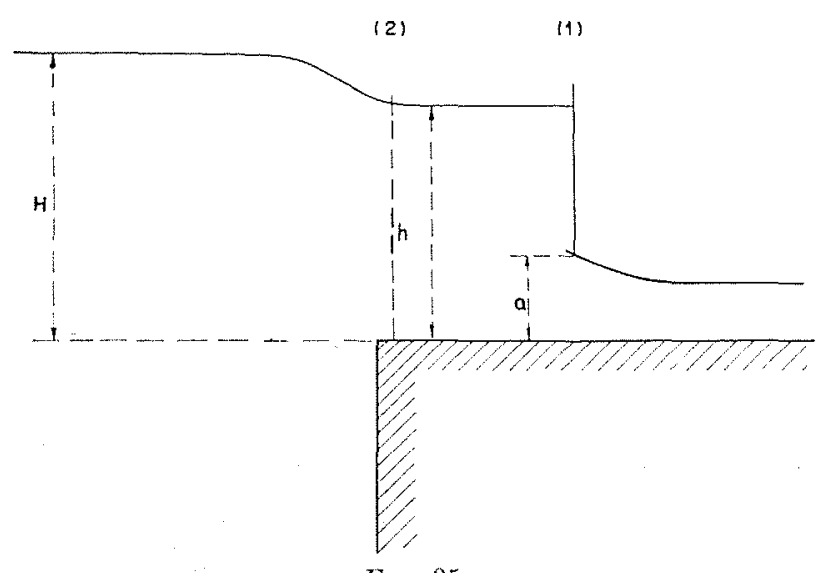

Fig. 25 ainsi que les pertes de charges par frottement entre (1) et (2).

Dans ces conditions, l'application des théorèmes précités nous permet de connaitre pour un débit $Q$ la valeur des fonctions $H(a)$ et $M(a)$ dans la section origine (1) du canal. On a, en effet :

$$
\begin{aligned}
& \mathrm{H}==h+\frac{\mathrm{Q}^{2}}{2 g l^{2} h^{2}}=\mathrm{H}(a) \\
& \quad \frac{h^{2}}{2}+\frac{Q^{2}}{g l^{2} h}=\mathrm{M}(a)+\frac{\mathrm{P}}{\mathrm{g} g}
\end{aligned}
$$

$h$ désignant la hauteur d'eau à l'amont de la vamne et $P$ la poussée de l'eau sur la face amont de celle-ci. Cette poussée est une fonction de l'ouverture $a$ et de la hauteur d'eau amont $h$ que nous préciserons plus loin.

Nous nous bornerons pour le moment à montrer que les conditions aux limites permettent Ia détermination du débit.

En éliminant $d^{2} y / d x^{2}$ entre les deux équations qui déterminent les fonctions $M$ et $H$, on obtient une équation donnant la pente de la ligne d'eau au droit de la vanne:

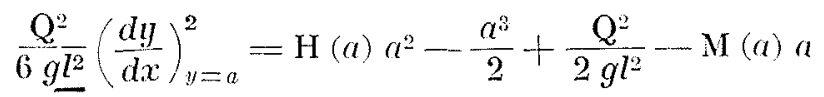

dont il faut prendre la solution correspondant à une pente négative.

Pour une valeur donnée du débit $Q$, la ligne d'eau est ainsi parfaitement déterminée par les conditions de CAuchy au droit de la vanne. 


$$
\begin{aligned}
& \text { Pour } x=0 \quad \begin{aligned}
y & =a \quad \mathrm{H}
\end{aligned}=\mathrm{H}(a): \\
& \frac{d y}{d x}=\left(\frac{d y}{d x}\right)_{y=a}
\end{aligned}
$$

Le débit est alors déterminé par les conditions d'écoulement aval.

Avant d'examiner l'infiuence de ces conditions, nous commencerons par étudier l'écoulement sous une vanne en négligeant le frottement, hypothèse sensiblement conforme à la réalité lorsque le radier, supposé horizontal, s'étend à l'aval sur une assez faible longueur.

ThéRIE DES ÉCOULEMENTS PAR VANNE DE fONDS.

Le frottement étant négligé, la ligne d'eau à l'aval de la vanne est une solution de l'équation différentielle du second ordre :

$$
\frac{\mathrm{Q}^{2}}{g l^{2}}\left[\frac{1}{3 y} \frac{d^{2} y}{d x^{2}}-\frac{1}{6 y)^{2}}\left(\frac{d y}{d x}\right)^{2}\right]=\mathrm{H}-y-\frac{\mathrm{Q}^{2}}{2 g l^{2} y^{2}}
$$

que l'on peuc mettre sous la forme:

$$
\left.\frac{\mathrm{Q}^{2}}{6 g l^{2}} \frac{d}{d y}\left[\frac{1}{!} \frac{d y}{d x}\right)^{2}\right]=\mathrm{H}-y-\frac{\mathrm{Q}^{2}}{2 g l^{2} y^{2}}
$$

$\mathrm{H}$ désignant la charge à l'amont de la vanne. C'est une constante caractérisant l'écoulement $\left(^{*}\right)$.

On en déduit immédiatement une intégrale première sous la forme:

$\frac{\mathrm{Q}^{2}}{6 g l^{2}}\left(\frac{d y}{d x}\right)^{2}=\mathrm{H} y^{2}-\frac{y^{3}}{2}+\frac{\mathrm{Q}^{2}}{2 g l^{2}}+\mathrm{C} y$

C étant une constante arbitraire qui est déterminée par la connaissance de la valeur de M. En effet, en se reportant au système (8) qui s'écrit :

$$
\left\{\begin{array}{l}
\frac{\mathrm{Q}^{2}}{3 g l^{2}}\left[\frac{d^{2} y}{d x^{2}}-\frac{1}{y}\left(\frac{d y}{d x}\right)^{2}\right]=\mathrm{M}-\frac{y^{2}}{2}-\frac{\mathrm{Q}^{2}}{g l^{2} y} \\
\frac{d \mathrm{M}}{d x}=0
\end{array}\right.
$$

on voit que la fonction $M(x)$ est également une constante caractéristique de l'écoulement et que la ligne d'eau est également une courbe intégrale de l'équation :

$\frac{\mathrm{Q}^{2}}{3 g l^{2}}\left[\frac{d^{2} y}{d x^{2}}-\frac{1}{y}\left(\frac{d y}{d x}\right)^{2}\right]=\mathrm{M}-\frac{y^{2}}{2}-\frac{\mathrm{Q}^{2}}{g l^{2} y}$

(*) En effet lorsqu'on néglige le frottement et si le radier est horizontal $i=0$, la seconde equation du systime (9) s'écrit $d \mathrm{H} / d x=0$, c'est-à-dire $\mathrm{H}=\mathrm{c}^{\mathrm{st} \text { e }}$, qui s’intègre aisément en posant $\left(\frac{d y}{d x}\right)^{2}=z(y)$, cc qui conduit à l'équation linéaire du premier ordre :

$$
\frac{\mathrm{Q}^{*}}{6 g l^{2}}\left[\frac{d z}{d y}-\frac{2 z}{y}\right]=\mathrm{M}-\frac{y^{2}}{2}-\frac{\mathrm{Q}^{2}}{g y l^{2}}
$$

dont l'intégration est immédiate par l'utilisation du facteur intégrant $1 / y y^{2}$ :

$$
\frac{Q^{2}}{6 g l^{2}} \frac{d}{d y}\left[\frac{z}{y^{2}}\right]=\frac{M}{y^{2}}-\frac{1}{2}-\frac{Q^{2}}{g y^{3} l^{2}}
$$

on obtient ainsi l'intégrale première :

$$
\left.\frac{\mathrm{Q}^{2}}{6 g l^{2}}\left(\frac{d^{\prime} y}{d x}\right)^{2}=-\mathrm{M} y-\frac{y^{\prime \prime}}{2}+\frac{\mathrm{Q}^{2}}{2 g^{2}}+\mathrm{C}_{1} y^{2} \cdot 19\right)
$$

Les équations (18) et (19) sont équivalentes, la forme (19) montrant que la constante d'integration $\mathrm{C}$ qui intervient dans (18) $\mathrm{C}=-\mathrm{M}$. Dans ces conditions, (18) et (19) s'écrivent:

$$
\frac{\mathrm{Q}^{2}}{6 g l^{2}}\left(\frac{d y}{d x}\right)^{2}=\mathrm{H} y^{2}-\frac{y^{3}}{2}+\frac{\mathrm{Q}^{2}}{2 g l^{2}}-\mathrm{M}_{y}
$$

Précisons maintenant les valeurs de $\mathrm{H}$ et $\mathrm{M}$ qui sont données, nous l'avons vu, par le théorème de Bernoulli et des quantités de mouvement.

Si $h$ est la hauteur d'eau à l'amont de la vanne (fig. 25 ), on a :

$$
\begin{aligned}
& \mathrm{H}=h+\frac{\mathrm{Q}^{2}}{2 g l^{2} h^{2}} \\
& \mathrm{~N}=\frac{h^{2}}{2}+\frac{\mathrm{Q}^{2}}{g l^{2} h}-\frac{\mathrm{P}}{l \circ g}
\end{aligned}
$$

La poussée de l'eau $P$ sur la face amont de la vanne est fonction de l'inclinaison et de la forme du taillant, de la hauteur d'eau amont $h$

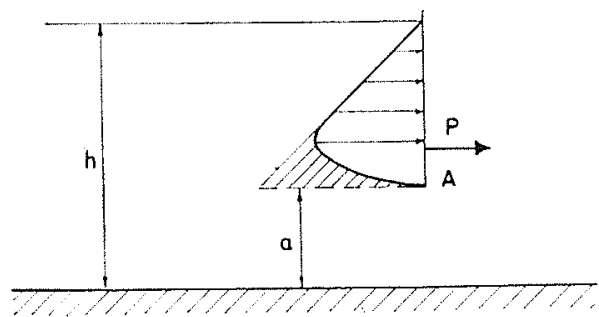

Frig. 26

et de l'ouverture $a$. Nous nous placerons délibérément dans l'hypothèse d'un taillant vertical à bord vif.

Nous mettrons la poussée par unité de longueur $\mathrm{P} / l$ sur la face amont sous la forme:

$$
\frac{\mathrm{P}}{l \rho g}=\frac{(h-a)^{2}}{2} \mathrm{~F}\left(\frac{\mathrm{Q}^{2}}{g l^{2} h^{2} a}\right)
$$


Lorsque $a=0$ (vanne fermée), la répartition des pressions est hydrostatique. On en déduit :

$$
\mathrm{F}(o)=1 \text {. }
$$

Pour une valeur faible de l'ouverture relative $a / h$, nous nous limiterons an premier terme du développement de :

$$
\begin{gathered}
\mathrm{F}\left(\frac{\mathrm{Q}^{2}}{g l^{2} h^{2} a}\right) \\
\mathrm{F}\left(\frac{a}{h}\right)=1 \cdots \frac{\mathrm{Q}^{2}}{g l^{2} h^{2} a}+\ldots
\end{gathered}
$$

y. étant un coefficient sans dimension dont nous déterminerons plus loin la valeur dans l'hypothèse d'un taillant vertical à bord vif. En tout état de cause, u. est nécessairement positif car la pression étant égale à la pression atmosphérique au droit du bord inférieur $A$ de la vanne, on doit avoir :

$$
\frac{\mathrm{P}}{l \bullet g}<\frac{(h-a)^{2}}{2}
$$

Les valeurs de $\mathrm{H}$ et $\mathrm{M}$ étant déterminées, la connaissance du débit $Q$ permet d'obtenir la ligne d'eau. C'est la courbe intégrale de l'équation différentielle (18) qui satisfait à la condition de Cauchy.

$$
x=0 \quad y=a
$$

En fait, ce sont les conditions d'écoulement aval qui déterminent le débit, ainsi que nous le verrons plus loin en étudiant, compte tenu du frottement, le cas d'un canal de pente $i$. Par analogie avec cette étude, nous ferons, pour calculer le débit, l'hypothèse suivante : «La ligne d'eau est la courbe intégrale particulic̀e de l'équation (18) qui admet une asymptote horizontale. »

Dans ces conditions, si $y=y_{0}\left(^{*}\right)$ est l'ordonnée de cette asymptote, l'équation en $y$ :

$$
\mathrm{H} y^{2}-\frac{y^{3}}{2}+\frac{\mathrm{Q}^{2}}{2 g^{2}}-\mathrm{M} y=0
$$

admet une racine double pour $y=y_{0}$, ce qui conduit aux deux relations :

$$
\left\{\begin{array}{l}
\mathrm{H} y_{0}{ }^{2}-\frac{y_{0}{ }^{3}}{2}+\frac{\mathrm{Q}^{2}}{2 g l^{2}}-\mathrm{M} y_{0}=0 \\
2 \mathrm{H} y_{0}-\frac{3 y_{0}^{2}}{2}-\mathrm{M}=0
\end{array}\right.
$$

(*) Ne pas confondre $y_{0}$ avec la hauteur normale qui n'est d'ailleurs pas défnie puisque $i=0$ et que l'on ne tient pas compte des frottements. d'où l'on déduit :

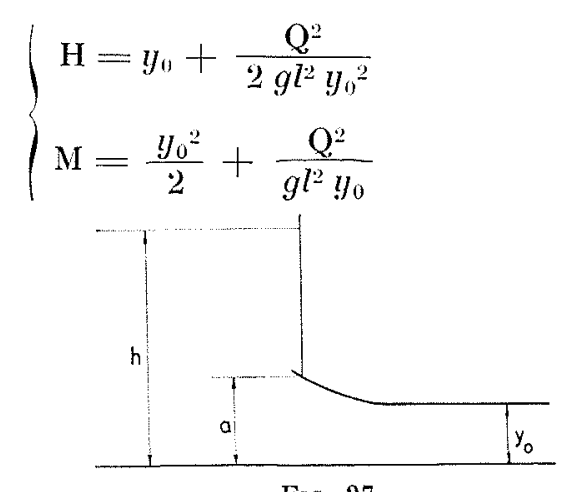

FIG. 27

ou, en tenant compte des valeurs de $\mathrm{H}$ et $\mathrm{M}$ :

$$
\begin{aligned}
& y_{0}+\frac{\mathrm{Q}^{2}}{2 g l^{2} y_{0}^{2}}=h+\frac{\mathrm{Q}^{2}}{2 g^{2} h} \\
& \frac{y_{0}^{2}}{2}+\frac{\mathrm{Q}^{2}}{g l^{2} y_{0}}=a h \frac{a^{2}}{2}+\frac{Q^{2}}{g l^{2} h}+\frac{Q^{2}(h-a)^{2}}{g^{2} h^{2} a}
\end{aligned}
$$

équations qui, pour une hauteur d'eau amont $h$ et une ouverture $a$ données, déterminent le débit $Q$ et la hauteur d'eau $y_{0}$ à l'aval.

Nous adopterons pour la résolution de ce système des variables sans dimensions en utilisant les coefficients classiques :

Ouverture relative : $\lambda=a / h$

Coefficient de contraction : $\sigma=y_{0} / a$.

La première équation s'écrit alors :

$$
\begin{gathered}
\mathrm{Q}^{2}=\frac{2 g l^{2} y_{0}^{2} h^{2}}{h+y_{0}}=2 g l^{2} a^{2} h \times \frac{\sigma^{2}}{1+\lambda \sigma} \\
\mathrm{Q}=\frac{\sigma}{\sqrt{1+\lambda \sigma}} l a \sqrt{2 g h}=\mathrm{C}_{q} l a \sqrt{2 g h}
\end{gathered}
$$

$\mathrm{C}_{q}=\frac{\sigma}{\sqrt{1+\lambda \sigma}}$ désignant le coefficient de débit.

En reportant la valeur de $Q^{2}$ dans la deuxième équation, on est conduit à une équation en $\sigma$ :

$$
\begin{aligned}
(1+\lambda \sigma)\left[\lambda \sigma^{2}-2+\lambda\right] & \\
& -2 \mu(1-\lambda)^{2} \sigma^{2}=4 \sigma(\sigma \lambda-1)
\end{aligned}
$$

qui donne le coefficient de contraction $\sigma$ en fonction de l'ouverture relative $\lambda=(a / h)$.

CAS D'UNe « OUVERTURE Relative» NULle $(\lambda=0)$.

Ce cas est réalisé lorsque la hauteur d'eau amont $h$ est grande par rapport à l'ouverture $a$ de la vanne. 
Le coefficient de débit $\mathrm{C}_{q m}$ est alors égal au coefficient de contraction $\sigma_{m}$ et $\sigma_{m}$ est solution de l'équation du second degré $-1+2 \sigma=y \cdot \sigma^{2}$.

Ce cas particulier est intéressant car il va nous permettre de déterminer la valeur de $\mu$ pour un taillant vertical à bord vif. En effel, lorsque $a / h$ est très petit, tout se passe comme si le fluide à l'amont de la vanne et loin de l'ouverture était à une pression uniforme. On est conduit au problème classique d'hydrodynamique de lécoulement par ane fente pour un fluide non pesant en mouvement irrotationnel.
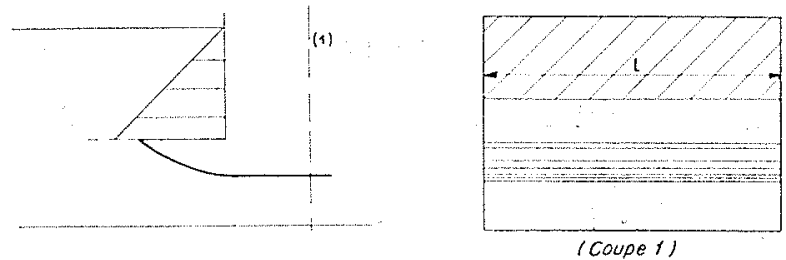

Fic. 28

Sa résolution à l'aide des fonctions analytiques conduit à des valeurs égales pour le coefficient de débit et le coefficient de contraction.

$$
c_{q m}=a_{m i}=\frac{\pi}{\pi+2}=0.611
$$

On en deduit la valeur du coefficient s.:

$$
\beta=\frac{1+2 \sigma_{m}}{\tau_{m}^{2}} \text { d'où } \mu=0,595
$$

Remarquons en passanl que dans le cas d'un orifice rentrant (orifice de BORDA), la répartition des pressions sur la lace amont de la ranne est rigoureusement hydrostatique; on a alors : $\because=0 \quad \mathrm{C}_{q}=\sigma=1 / 2$ dans l'hypothèse d'une ouverture relative très petite. Pour une ouverture relative $\lambda$ différente de 0 , le coefficient de contraction serait donnée par l'équation (20) où l'on fait $y=0$.

Revenant au cas d'un tailant vertical à bord vif, le coefficient de contraction $\sigma$ est donne en fonction de l'ouverture relative par l'équation :

$$
\begin{gathered}
(1+\lambda \sigma)\left[\lambda \sigma^{2}-2+\lambda\right]-1,190(1-\lambda)^{2} \sigma^{2} \\
=4 \sigma(\sigma \lambda-1)
\end{gathered}
$$

dont il convient de prendre la solution inférieure à 1 .

Le tableau ci-après donne les valeurs de o et $\mathrm{C}_{17}$ en fonction du rapport $\lambda=(a / h)$.

L'examen de ce tableau montre que le coefficient de débit $C_{q}$ reste sensiblement constant lorsque 2 varie de 0 à 0,5 .

\begin{tabular}{|c|c|c|c|c|c|c|c|}
\hline$i_{c}=\frac{a}{h}$ & 0 & 0,1 & 0,2 & 0,3 & 0,4 & 0,5 & 1 \\
\hline$\sigma$ & 0,611 & 0,629 & 0,649 & 0,670 & 0,693 & 0,718 & 1 \\
\hline $\mathrm{C}_{g}$ & 0,611 & 0,610 & 0,6105 & 0,611 & 0,613 & 0,616 & 0,707 \\
\hline
\end{tabular}

ce qui est conforme à l'expérience. Pour des vaJeurs supérieures du rapport $a / h$, on pent se demander s'il est justifiable de se limiter dans l'expression de la poussée :

$\mathrm{P}=g l \frac{(h-a)^{2}}{2} \mathrm{~F}\left(\frac{\mathrm{Q}^{2}}{g l^{2} h^{2} a}\right)$ au premier terme du développement de $\mathrm{F}\left(\frac{\mathrm{Q}^{2}}{g^{2} h^{2} a}\right)$ :

$$
\mathrm{F}\left(\frac{\mathrm{Q}^{2}}{g l^{2} h^{2} a}\right)=1-0,595 \frac{\mathrm{Q}^{2}}{g^{2} h^{2} a}+\ldots
$$

En fait, lorsque $a / h$ est suffisamment grand, Pinfluence de la poussée devient négligeable.

On voit aussitôt que lorsque $\lambda$ tend vers 1 , la racine de l'équation en $\sigma$ inf'érieure à 1 tend vers l'unite, quel que soit $\mathrm{F}\left(\frac{\mathrm{Q}^{2}}{g l^{2} h^{2} a}\right)$, on at alors : $\sigma=1 \quad \jmath=1$.

$$
C_{t t}=\frac{\sigma}{\sqrt{1+\lambda \sigma}}=\frac{1}{\sqrt{2}}=0,707 \text {. }
$$

$$
\mathrm{Q}^{2}=\mathrm{C}_{a^{2}} l^{2} a^{2} 2 g h \quad \mathrm{Q}^{2}=l^{2} a^{2} g h \quad y_{0}=a=h
$$

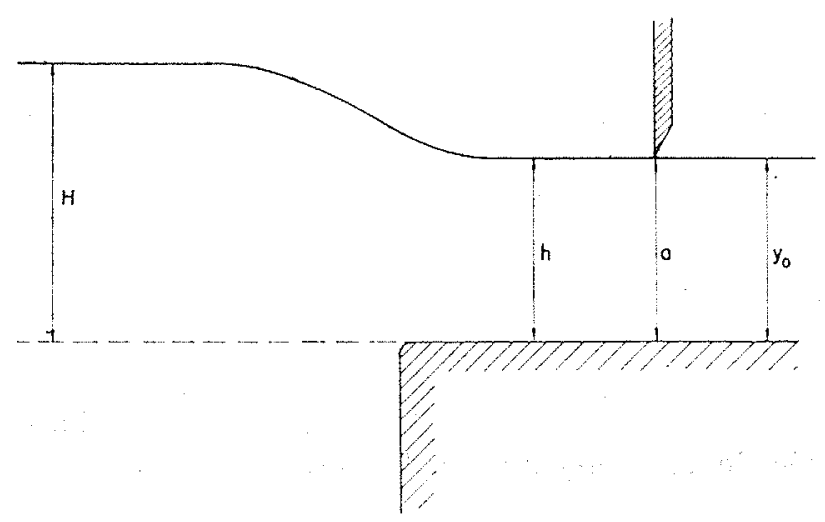

Fig. 29

d'où :

$$
\begin{gathered}
\frac{\mathrm{Q}^{2}}{g l^{2} h}=1 \\
\mathrm{H}=h+\frac{\mathrm{Q}^{2}}{2 g l^{2} h^{2}}=h+\frac{h}{2}=\frac{3}{2} h
\end{gathered}
$$

La hauteur d'eau $h$ est égale à la hauteur critique. On retrouve la formule de Belanger donnant le débit d'un déversoir à large senil:

$$
\mathrm{Q}=\frac{1}{\sqrt{3}} \cdot \frac{2}{3} l \mathrm{H} \sqrt{2 g \mathrm{H}}
$$


Equation de la ligNe d'EAU.

L'équation différentielle de la ligne d'eau (18) se met sous la forme :

$$
\frac{\mathrm{Q}^{2}}{3 g l^{2}}\left(\frac{d y}{d x}\right)^{2}=\left(y-y_{0}\right)^{2}\left[\frac{\mathrm{Q}^{2}}{g l^{2} y_{0}^{2}}-y\right](17)
$$

en faisant intervenir l'ordonnée $y_{0}$ de l'asymptote horizontale.

Cette équation à variables séparées s'intègre à l'aide des fonctions élémentaires.

L’intégrale générale s'écrit :

$$
y=y_{0}+4\left(\frac{\mathrm{Q}^{2}}{g l^{2} y_{0}^{2}}-y_{0}\right) \frac{\mathrm{A} e^{\alpha x / y_{0}}}{\left(1+\mathrm{A} e^{\alpha x / y_{0}}\right)^{2}}
$$

a étant un coefficient sans dimension dont la valeur est :

$$
x=\frac{\sqrt{3\left(Q^{2}-g l^{2} y_{0}^{3}\right)}}{Q}
$$

La constante d'intégration s'obtient en écrivant que pour $x=0 \quad y=a$.

$$
a=y_{0}+4\left(\frac{\mathrm{Q}^{2}}{g y_{0}^{2}}-y_{0}\right) \frac{\mathrm{A}}{(1+\mathrm{A})^{2}}
$$

En faisant intervenir les variables sans dimensions :

$$
\lambda=\frac{a}{h} \quad \sigma=\frac{y_{0}}{a}
$$

A est la solution de l'équation du second degré :

$(1-\sigma) \mathrm{A}^{2}+2\left(1+\sigma-\frac{4}{\lambda(1+\lambda \sigma)}\right) \mathrm{A}+1-\sigma=0$

dont il faut prendre la racine supérieure à l'unité, qui correspond à une pente négative de la ligne d'eau au point A $x=0 \quad y=a$.

\section{Profil OPTIMUM}

DE LA SURFACE D'APPUI D'UNE VANNE.

Les vannes courantes ne peuvent être constituées par un taillant à bord vif, leur surface d'appui doit avoir une certaine épaisseur de facon à assurer la transmission des efforts. Dans ces conditions si, pour une certaine ouverture relative $a / h$, la ligne d'eau est située au-dessous du profil de la surface d'appui, il se produit entre cette surface et le niveau du courant un espace en forme de coin où l'air est entraîné par le frottement hors de cet espace. La dépression ainsi créée est la cause de phénomènes pulsatoires avec décollements et recollements successifs de la lame d'eau avec la surface d'appui. Les effets dynamiques correspondants engendrent dans certains cas des vibrations particulièrement dangereuses du corps de la vanne.

Désignons par $a_{1}$ l'ouverture maximum de la vanne correspondant à la hauteur d'eau amont

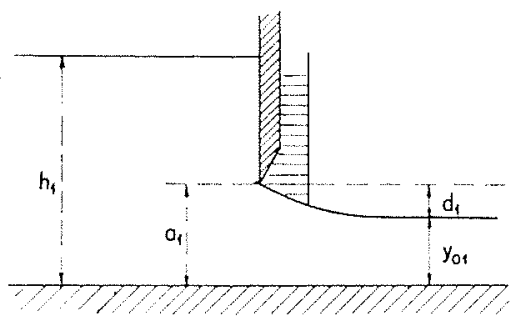

Fic. 30

maximum $h_{1}$. Soit, dans ces conditions, $\left(\mathbf{E}_{1}\right)$ la courbe d'écoulement théorique correspondant à un taillant vertical à bord vif. On ne change rien à l'écoulement en remplaçant le taillant par un profil épousant une partie de la courbe $\left(\mathbf{E}_{1}\right)$.

$\mathrm{Si}$, pour une ouverture quelconque $a$ inférieure à $a_{1}$, la courbe théorique d'écoulement (E) est située au-dessous de $\left(\mathrm{E}_{1}\right)$, la lame d'eau correspondant à cette position exercera sur la surface d'appui une poussée verticale dirigée de bas en haut, et aucun phénomène pulsatoire ne sera à craindre au cours de la levée de la vanne.

Une condition nécessaire pour qu'il en soit ainsi se déduit de l'examen de la figure 31 .

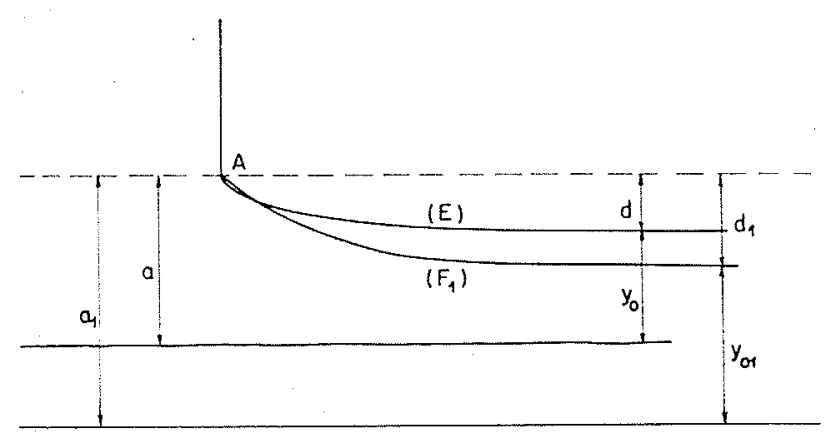

Fig. 31

Elle s'écrit :

$$
d=a-y_{0}<d_{1}=a_{1}-y_{01}
$$

La manœuvre de levée de la vanne ayant lieu sous la charge constante :

$$
\mathrm{H}=h+\frac{\mathrm{Q}^{2}}{2 g l^{2} h^{2}}=h_{1}+\frac{\mathrm{Q}_{1}^{2}}{2 g l^{2} h_{1}^{2}}
$$


nous sommes conduit à poser :

$$
\begin{aligned}
d=a-y_{0} & =h \lambda(1-\sigma) \\
\mathrm{H}=h+\frac{\mathrm{Q}^{2}}{2} g l^{2} h^{2} & =h\left(1+\frac{\lambda^{2} \sigma^{2}}{1+\lambda \sigma}\right)
\end{aligned}
$$

d'où :

$$
d=\frac{\lambda(1-\sigma)}{1+\frac{\lambda^{2} \sigma^{2}}{1+\lambda \sigma}} \mathrm{H}
$$

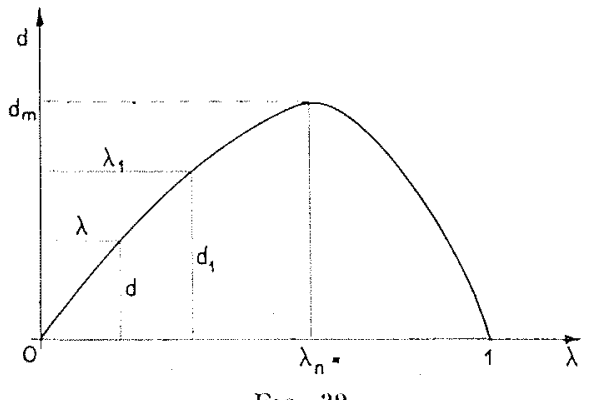

Fic. 32

L'examen de la courbe représentant la variation de $d$ lorsque l'ouverture relative $\lambda$ varie de 0 à 1 ( $\sigma$ étant relié à $\lambda$ par la relation (20)), montre que $d$ passe par un maximum $d_{m}$ correspondant à une onverture relative $\lambda_{m}$.

La condition $d<d_{1}$ exige donc que :

$$
\lambda_{1}=a_{1} / h_{1}<\lambda_{m}
$$

Cetle condition n'est d'ailleurs pas suffisante. En effet, la pente de la tangente en $A$ à la courbe (E) est donnce, en fonction des variables sans dimensions, par l'expression :

$$
\left(\frac{d y}{d x}\right)_{y=a}=\frac{\sqrt{3}}{\sigma}(1-\sigma) \sqrt{1-\frac{\lambda(1+\lambda \sigma)}{2}}
$$

Lorsque l'ouverture relative $\lambda$ croit de 0 à 1 , cette pente décroît de 1,10 à 0 . La courbe (E) se trouve donc au voisinage du point $A$ audessous de la courbe $\left(\mathbb{E}_{1}\right)$, même si la condition précédente est réalisée (confers fig. 31).

Il convient donc de s'assurer que, pour une onverture $a$ inférieure à l'ouverture maximum $\alpha_{1}$, la courbe $(\mathrm{E})$ recoupe $\left(\mathrm{E}_{1}\right)$ à l'amont de l'extrémité du profil de la vanne.

EQvation de la Ligne d'EAU POUR UNE OUVERTURE RELATIYE NULLE.

C'est le cas où la hauteur d'eau amont $h$ est tris grande vis-à-vis de l'ouverture de la vanne.
L'équation différentielle (17) de la ligne d'eau s'écrit, en variables sans dimensions :

$$
\begin{gathered}
u=(y / a) \quad t=(x / a): \\
\left(\frac{d u}{d t}\right)^{2}=\frac{3}{\sigma^{2}}(u-\sigma)^{2}\left[1-\frac{\lambda}{2}(1+\lambda \sigma) u\right]
\end{gathered}
$$

Pour $\lambda=0$, la détermination négative s'écrit:

$$
\frac{d u}{d t}=-\frac{\sqrt{3}}{\sigma}(u-\sigma)
$$

dont l'intégrale générale s'écrit :

$$
u=\sigma+A e^{-(-(\pi) / n) t}
$$

A étant déterminé par la condition :

$$
t=0 \quad u=1,
$$

on en déduit l'équation de la ligne d'eau sous la forme:

$$
\frac{y}{a}=\sigma+(1-\sigma) e^{-(\sqrt{3} / a)(x / a)}
$$

ou, en tenant compte de la valeur du coefficient de contraction pour :

$$
\begin{gathered}
y=0 \quad \sigma=0,611 \\
\frac{y}{a}=0,611+0,389 e^{-2,85.5 \% 12}
\end{gathered}
$$

\section{VANNE SUIVE D'UN CANAL RECTANGULAIRT DJE PENTE UNIFORME.}

Nous supposerons la vanne constituée par un taillant vertical à bord vif et nous tiendrons compte du frottement.

Nous avons vu que, dans ces conditions, le debit est déterminé par les conditions d'écoulement à l'aval de la vanue. Il dépend donc non seulement de l'ouverture relative $a / h$, mais aussi de l'inclinaison du radier,

La ligne de remous à l'aval de la vanne sera déterminée par la courbe (C) qui la représente dans le plan $\mathrm{HOy}$.

Les fonctions $\mathrm{H}(\alpha)$ et $\mathrm{M}(\alpha)$ à l'aplomb de la vanne $(x=0)$ sont données, en fonction du débit $Q$, par les relations:

$$
\begin{aligned}
& \mathrm{H}(a)=h+\frac{\mathrm{Q}^{2}}{2 g l^{2} h^{2}}=7 e \\
& \mathrm{M}(a)=a h-\frac{a^{2}}{2}+\frac{\mathrm{Q}^{2}}{g l^{2} h}+\cdots \frac{\mathrm{Q}^{2}}{2 g l^{2} h^{2} a}(h-a)^{2}
\end{aligned}
$$


d'où l'on déduit la pente de la ligne d'eau en $A$ :

$\frac{Q^{2}}{6 g l^{2}}\left(\frac{d y}{d x}\right)_{y=1}^{2}=\mathrm{H}(a) a^{2}-\frac{a^{3}}{2}+\frac{\mathrm{Q}^{2}}{2 g l^{2}}-\mathrm{M}(a) a$ soit :

$$
\left(\frac{d y}{d x}\right)_{t=0}^{2}=3\left(1-y_{0}\right)\left(1-\frac{a}{h}\right)^{2}
$$

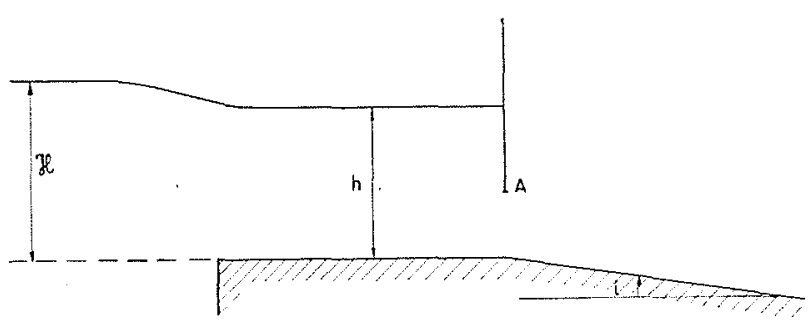

Fic. 34

La courbe (C), qui représente la ligne de remous dans le plan $\mathrm{HOy}$, est done la courbe intégrale du systeme (10) $\mathrm{H}=\mathrm{H}(\mathrm{y})$ :

$$
\left\{\begin{array}{l}
\frac{\mathrm{Q}^{2}}{6 g l^{2}} \frac{d}{d y}\left[-\frac{z^{2}}{y}\right]=\mathrm{H}-y-\frac{\mathrm{Q}^{2}}{2 g l^{2} y^{2}} \\
z \quad \frac{d \mathrm{H}}{d y}=i-\frac{\mathrm{Q}^{2}}{c^{2} l^{2} y^{3}}
\end{array}\right.
$$

qui salisfait pour $y=a$ aux conditions de Cavchy :
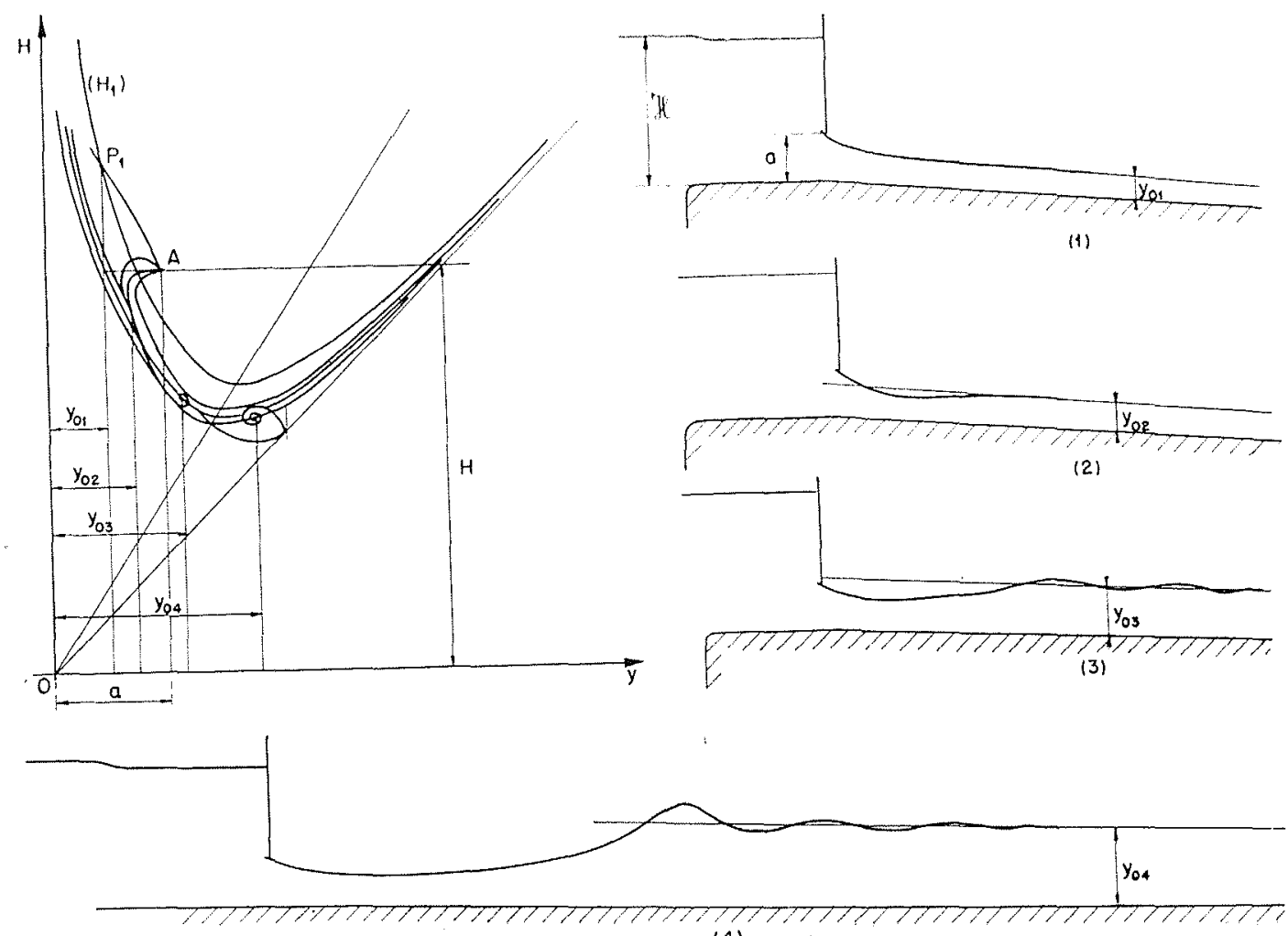

(4)

FIG. 35

$$
\begin{aligned}
& H(a)=\mathscr{H} \\
& z(a)=\left(\frac{d y}{d x}\right)_{y=a}
\end{aligned}
$$

Pour un débit donné $Q$, cette courbe (C) est donc parfaitement déterminée. Il reste à déterminer le débit $Q$ par les conditions d'écoulement aval, c'est-à-dire en écrivant que la ligne de remous est asymptote à la droite du regime uniforme lorsque $x$ tend vers $\infty$. Or, on sait qu'aux lignes de remous satisfaisant à cette condition correspond dans le plan (HOy) une simple infinité de courbes (C). Il existe done entre les données initiales, $a, \mathrm{H}(a), z(a)$, une relation qui détermine le débit.

Le tracé de la courbe de remous se déduit de l'allure de la courbe (C) dans le plan HOy. Tes courbes de la figure 35 donnent le tracé des diverses lignes d'eau et des lignes (C) correspondantes, pour une charge amont $H$ donnée lorsqu'on fait varier la pente du canal de décharge.

Nos équations ne sont applicables sous la forme (10) que si la pente est suffisamment faible pour qu'on puisse poser $\sin i=i, \quad \cos i=1$.

Dans ces conditions, la pente de la tangente ('n $\mathrm{A}$ à la courbe $(\mathrm{C})$ :

$$
\left(\frac{d \mathrm{H}}{d !}\right)_{y=a}=\left[i-\frac{Q^{2}}{c^{2} l^{2} a^{3}} \mid \frac{1}{\left(\frac{d y}{d x}\right)_{y=a}}\right.
$$

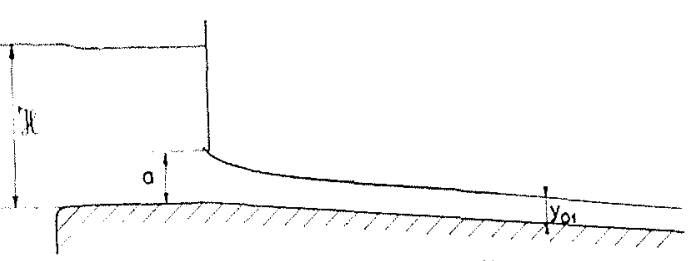

(1)

(2)

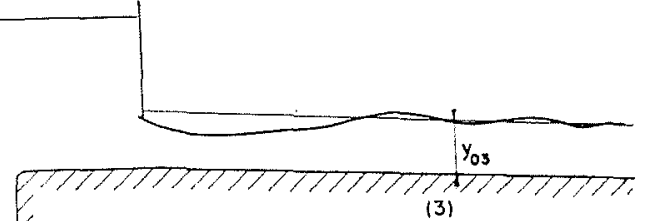

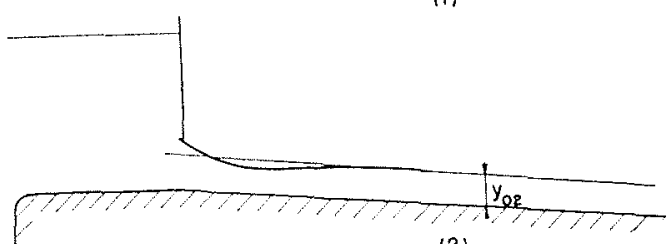


Comme la pente de la tangente en $A$ à la courbe de remous $(d y / d x)_{y=a}$ ne dépend que du rapport $a / h$ et est voisin de 1 en valeur absolue, si $a / h$ est assez grand $(d \mathrm{H} / d y)_{y=a}$ a une valcur faible; $(d y / d x)_{y=a}$ étant négatif $(d \mathrm{H} / d y)_{y=a}$ a un signe variable avec la quantité $i-\left(Q^{2} / c^{2} l^{2} a^{3}\right)$, c'està-dire avec la position du point $A$ par rapport à la droite (D) du régime uniforme $y=y_{0}$, cette pente est négative si $y_{0}<a$ et positive si $y_{0}>a$.

Pour une valeur suffisamment faible de la pente du canal de décharge, le régime devient lluvial dans le canal, on voit apparaitre un ressaut ondulé. Lorsque la pente diminue encore, le front de l'onde se raidit et la première vague déferle, le ressaut remonte progressivement dans le canal et vient recouvrir le bord inférieur de la vanne.

Pour une valeur faible de la pente du canal de décharge et lorsque le seuil n'est pas noyé, il est intéressant de comparer la valeur du débit et la forme de la courbe de remous au voisinage de la vanne au débit théorique $Q_{T}$ et à la ligne de remous théorique $\left(R_{\mathrm{T}}\right)$ précédemment obtenus en supposant le radier horizontal ef en négligeant le frottement.

Cette courbe $\left(R_{T}\right)$ est la courbe intégrale de l'équation différentielle :

$$
\frac{\mathrm{Q}^{2}}{6 g l^{2}} \frac{d}{d y}\left[\frac{1}{y}\left(\frac{d y}{d x}\right)^{2}\right]=\not{C}--y-\frac{\mathrm{Q}^{2}}{2 g l^{2} y^{2}}
$$

qui satisfait pour $x=0$ aux conditions de CAvcrY $y=a$ :

$$
\left(\frac{d y}{d x}\right)_{y=a}=-\sqrt{3(1-u)\left(1-\frac{a}{h}\right)}
$$

le débit théorique $Q_{\mathrm{T}}$ étant précisément la valeur qu'il faut donner à $Q$ pour que cette courbe admette une asymptote horizontale.

Dans le plan HOy, cette courbe $\left(\mathrm{R}_{\mathrm{T}}\right)$ est représentée par le segment $A B$ de la droite $\mathrm{H}=\mathscr{F}$, compris entre les droites d'équations $y=a, y=\sigma a$ ( $\sigma$ coefficient de contraction).

Lorsqu'on tient compte du frottement, la courbe de remous ( $R$ ) est la solution du système :

$$
\left\{\begin{array}{l}
\frac{\mathrm{Q}^{2}}{6 g l^{2}} \frac{d}{d y}\left[\frac{1}{y}\left(\frac{d y}{d x}\right)^{2}\right]=\mathrm{H}-y-\frac{\mathrm{Q}^{2}}{2 g l^{2} y^{2}} \\
\frac{d \mathrm{H}}{d x}=i-\frac{\mathrm{Q}^{2}}{c^{2} l^{2} y^{3}}
\end{array}\right.
$$

qui satisfait, pour $x=0$, aux conditions de CALCHY:

$$
y=a, \quad \mathrm{H}=\mathcal{H}, \frac{d y}{d x}=-\sqrt{3(1 \cdots \cdots,-j)}\left(1-\frac{a}{h}\right)
$$

Le débit réel $Q_{R}$ étant la valeur qu’il faut donner à $\mathrm{Q}$ pour que cette courbe intégrale soit asymptote à la droite $y=y_{0}$ du régime uniforme, $y_{0}$ étant défini par l'équation :

$$
i-\frac{\mathrm{Q}_{\mathrm{R}}^{2}}{c^{2} l^{2} y^{3}}=0
$$

Supposons que l'on donne précisément à la pente du canal de décharge la valeur :

$$
i_{12}=\frac{Q_{\mathbf{R}^{2}}}{c^{2} l^{2} \sigma^{3} a^{\prime \prime}}
$$

pour laquelle la hauteur du régime uniforme $y_{0}$ cö̈ncide avec $\sigma_{n}$.

Dans ces conditions, la courbe $\left(C_{R}\right)$, qui représente dans le plan $\mathrm{HOy}$ la ligne de remous (R), est voisine du segment $A B$ (fig. 36). $Q_{R}$ est donc
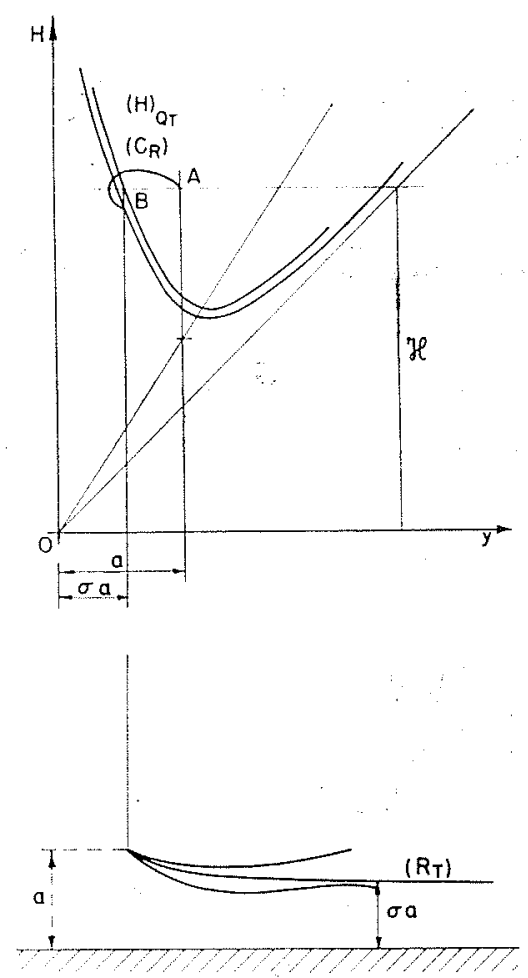

FIG. 36

pratiquement égal à $Q_{\mathrm{T}}$, en fait il lui est légèrement inférieur.

Quant au coefficient de contraction, il est dans ce cas légèrement inférieur au coefficient théorique $\sigma$, la hauteur d'eau remontant ensuite pour atteindre le niveau normal.

Lorsque la pente du canal de décharge est inférieure à $i_{R}$, le débit est inférieur à $Q_{T}$ et le coefficient de contraction supérieur au coefficient de contraction théorique.

Lorsque $i>i_{R}, Q$ est supérieur à $Q_{\mathrm{T}}$.

En conclusion, pour de faibles pentes du ca- 
nal de décharge, le débit varie entre des limiles assez voisines tant que le seuil n'est pas noyé, la valeur moyenne du débit élant bien représentée par le débit théorique $Q_{1}$.

\section{4. -- Déversoir à large seuil.}

Comme derniere application de nos équalions, nous étudierons un type d'écoulement rapidement varié qui a donné lieu à de nombreuses recherches théoriques et expérimentales: l'écoulement sur un déversoir à large seuil.

Nous supposerons le radier horizontal ot $l e$

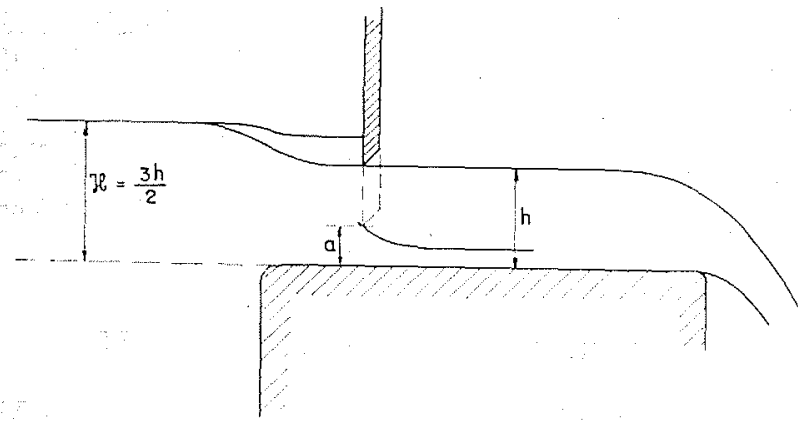

Fis. 37

réservoir amont suffisamment profond pour qu'on puisse négliger la vitesse d'approche. Nous designerons par ye la hauteur d'eau amont audessus du déversoir.

Nous commencerons par étudier le phénomene en négligeant le frottement.

\section{CAS D'Un SEUL Trìs LISSl:}

Dans ces conditions, nous avons vu au paragraphe précédent que si on règle le débit sur le seuil à l'aide d'une vanne d'ouverture $a$, pour une ouverture de la vanne égale aux $2 / 3$ de la hauteur d'eau à l'amont du seuil $a=h=\%$ re, la ligne d'eau effleurant le bord inférieur de la vanne était constituée par une droite horizontale.

Le débit a alors la valeur :

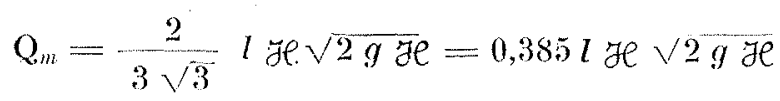

Ce résultat peut être établi d'une maniere différente en recherchant les conditions que doivent romplir les données pour que la ligne d'eau soit précisément une droite.

Cette ligne d'eau est une courbe intégrale do l'équation différentielle :

$$
\frac{\mathrm{Q}^{2}}{6 g^{2}}\left(\frac{d y}{d x}\right)^{2}=\mathscr{H} y^{2 \prime}-\frac{y^{3}}{2}+\frac{\mathrm{Q}^{2}}{2 g^{2}} \mathrm{M} y
$$

Eerivons que poux $y=h$ :

$$
\frac{d y}{d x}=\frac{d z y}{d x=0}=0
$$

On obtient :

$$
\begin{aligned}
& y e=\frac{Q^{2}}{2 g l^{*} h^{2}} \\
& M=\frac{h^{2}}{2}+\frac{Q^{2}}{g l^{2} h}
\end{aligned}
$$

L'équation précédente se met sous la forme:

$$
\left.\frac{\mathrm{Q}^{\prime}}{3 g l^{2}}\left(\frac{d y}{d x}\right)^{2}=(y) h\right)=\left(\frac{\mathrm{Q}^{2}}{g^{2} h^{2}} \cdot y\right)(17)
$$

dont lallure des courbes integrales est figuree sur la figure 38.

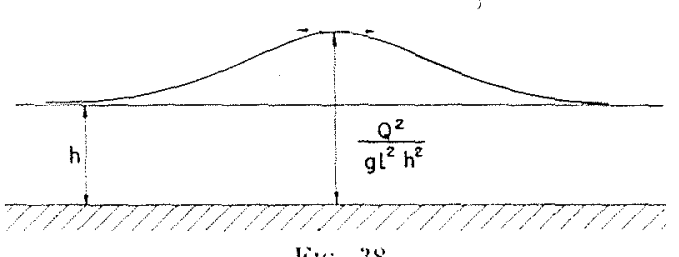

Lal condition $\frac{d y}{d x}=\frac{d^{2} y}{d x^{2}}=0$ pour $y=h$ n'est done pas suffisante pour que la ligne d'eau soil une droite, il faut en outre que $h=\frac{\mathrm{Q}^{2}}{g^{2} h^{2}}$ On en déduit :

$h=2 \frac{\not C}{3}, Q=Q_{m}=\frac{2}{3 \sqrt{3}} l \mathcal{H} \sqrt{2} g \mathscr{H}, \mathrm{M}=2 \frac{7 e^{-}}{3}$

En pratique, les conditions d'écoulement aval (hauteur d'eau dans le réservoir aval) et le frottement sur le seuil diminuent la valeur du débit qui est inférieur à $Q_{m}$, sauf dans l'hypothèse d'un seuil lisse dont la longueur n'excède pas quatre à cinc fois la hauteur d'eau amont.

Même si, dans la détermination de l'allure de la ligne d'eau, on peut en première approximation négliger le frottement et supposer la charge constante et égale à $\mathscr{J}$, on est conduit à prendre comme valeur du débit sur le seuil une quantité $\mathrm{Q}$ inférieure à $\mathrm{Q}_{m}$.

Dans ces conditions, la ligne d'eau est me courbe intégrale de l'équation (18).

$$
\frac{\mathrm{Q}^{2}}{6 g l^{2}}\left(\frac{d y}{d x}\right)^{2}=7 e y^{2}-\frac{y^{3}}{2}+\frac{\mathrm{Q}^{2}}{2 g}-\mathrm{M} y
$$

Les considérations qui vont suivre ont pour but de déterminer, pour une charge amont $\not \subset$ et un débit $Q$ sur le scuil, la hauteur d'eau $h:$ lextrémité amont et la valeur de la constante $M$. 
DÉtermination de la hauteur D'EaU $h$ A L'EXTRÉMITÉ AMONT DU SEUIL.

Pour une valeur de débit $\mathrm{Q}<\mathrm{Q}_{m}$, la hauleur d'eau sur le seuil oscille entre deux valeurs extrêmes $y_{0}$ et $y_{1}$ qui sont les racines les plus grandes de l'équation du troisième degré :

$$
\text { ge } y^{2}-\frac{y^{3}}{2}+\frac{\mathrm{Q}^{2}}{2 g}-\mathrm{M} y=0
$$

Le point le plus bas d'ordonnée $y=y_{0}$ est atteint nous le verrons, à une distance faible de l'extrémité amont du seuil.
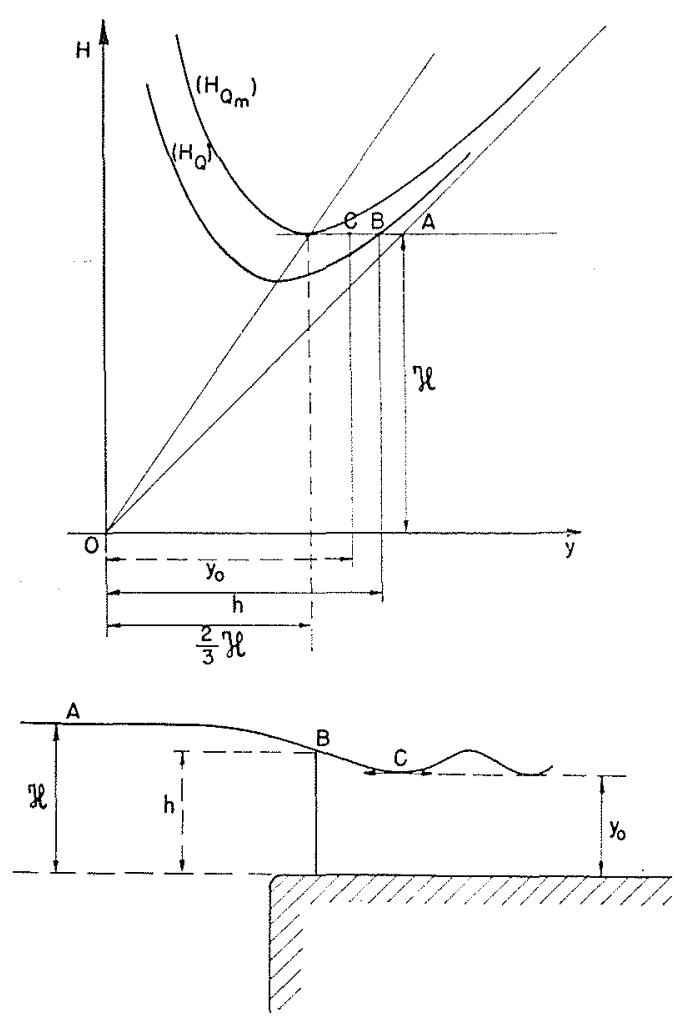

FIG. 39

Le point $\mathrm{C}$ de coordomnées $y=y_{0} \quad \mathrm{H}=\not{Z}$ qui le représente dans le plan $\mathrm{HOy}$, est situé audessus de la courbe $\left(\mathrm{H}_{2}\right)$ définie par l'équation :

$$
\mathrm{H}=y+\frac{\mathrm{Q}^{2}}{2 g l^{2} y^{2}}
$$

ce qui se déduit immédiatement de la relation :

$\frac{\mathrm{Q}^{2}}{3 g l^{2}}\left[\frac{1}{y} \frac{d^{2} y}{d x^{2}}-\frac{1}{2 y^{2}}\left(\frac{d y}{d x}\right)^{2}\right]=7 e-y-\frac{\mathrm{Q}^{2}}{2 g l^{2} y^{2}}$

En effet, pour :

$$
y=y_{0}, \quad \frac{d y}{d x}=0 \quad \frac{d^{2} y}{d x^{2}}>0
$$

done :

$$
\mathscr{H}-y_{0}-\frac{\mathrm{Q}^{2}}{2 g l^{2} y_{0}{ }^{2}}>0
$$

Il existe donc à l'amont de $\mathrm{C}$ un point $\mathrm{B}$ de la ligne d'eau correspondant à une hauteur' d'eau $h$, pour lequel on a rigoureusement :

$$
\mathscr{H}=h+\frac{\mathrm{Q}^{2}}{2 g l^{2} h^{2}}
$$

en ce point :

$$
\left(\frac{d^{2} y}{d x^{2}}\right)_{y=h}=\frac{1}{2 h}\left(\frac{d y}{d x}\right)_{y=h}^{2}
$$

Le point $B$ ne coüncide pas avec l'extrémité amont du déversoir, mais il en est très voisin. II est donc justifié de prendre pour hauteur d'eau $h$ à l'extrémité amont la racine de l'équation $\mathscr{H}=h+\left(\mathrm{Q}^{2} / 2 g l^{2} h^{2}\right)$ qui correspond au régime fluvial, racine qui est réelle pour toute valeur de $Q$ inférieure à $Q_{m}$.

\section{DÉTERminatron dE LA CONSTANTE $M$.}

La signification mécanique de la constante $M$ dans l'équation (18) a déjà été définie au paragraphe précédent. Elle s'introduit par application du théorème des quantités de mouvement et conduit, lorsqu'on néglige le frottement, à la relation :

$\frac{\mathrm{Q}^{2}}{3 g l^{2}}\left[\frac{d^{2} y}{d x^{2}}-\frac{1}{y}\left(\frac{d y}{d x}\right)^{2}\right]=\mathrm{M}-\frac{y^{2}}{2}-\frac{\mathrm{Q}^{2}}{g l y}$

dont l'intégrale première est, nous l'avons déjà démontré, équivalente à (18).

On est donc conduit, pour déterminer la valeur de $\mathrm{M}$, à appliquer le théorème des quantilés de mouvement au volume de fluide compris entre les sections (1), (2) et (3) représentées sur la figure 40 .

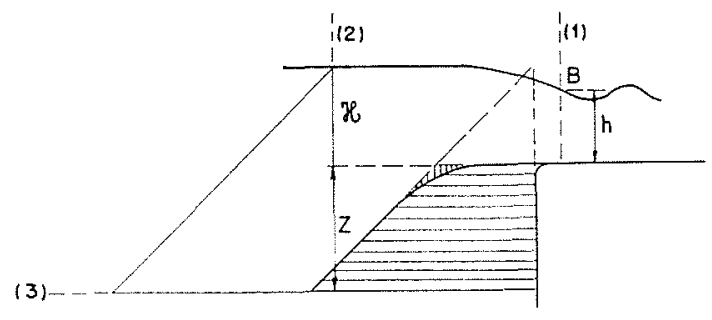

FIG. 40

(1) est la section précédemment définie où la hauteur d'eau est $h$ et qui coïncide pratiquement avec l'extrémité amont du déversoir;

(2) est une section normale situce suffisamment à l'amont pour que la vitesse de l'eau soit négligeable dans toute la section;

(3) est une section horizontale situé à une 
cote $z$ au-dessous du seuil, assez bas pour que la pression puisse être considérée comme constante et la vitesse nulle en tout point de cette section.

Dans ces conditions, le théorème des quantités de mouvement en projection sur un axe horizontal s'écrit :

$$
\frac{(\mathscr{H}+z)^{2}}{2}=\frac{1}{\rho g l} \iint_{(1)} p d \sigma+\frac{1}{l g} \iint_{(1)} u^{2} d \sigma+\frac{\mathrm{P}}{\rho g l}
$$

D'après la signification de $\mathrm{M}$, on $\mathbf{a}$ :

$$
\mathrm{M}=\frac{1}{\rho g l} \iint_{\{1\}} p d \sigma+\frac{1}{l g} \iint_{(1)} u^{2} d \sigma
$$

P désigne la poussée de l'eau sur la paroi amont du déversoir entre la section (3) et le seuil. Elle est représentée sur la figure 40 par l'aire hachurée horizontalement. On voit immédiatement, en se reportant à la figure 40 , que :

$$
\frac{\mathrm{P}}{l \odot g}=\frac{(\mathscr{H}+z)^{2}}{\mathbf{2}}-\frac{\mathscr{H}^{\mathscr{2}}}{\mathbf{2}}-\delta
$$

o étant une qualité positive qui dépend de la vitesse d'appel de l'eau au voisinage du seuil et qui est représentée sur la figure 40 par l'aire hachurée verticalement.

On en déduit :

$$
\mathrm{M}=\frac{\mathfrak{H}^{2}}{2}+\delta
$$

$\delta$ est une fonction de la vitesse à l'extrémité amont du seuil dont on peut déterminer la valeur lorsque le débit est nul et lorsqu'il prend sa valeur maximum $\mathrm{Q}_{m}$.

Le débit est nul lorsque le plan d'eau dans le réservoir aval est à la cote fo au-dessus du seuil.

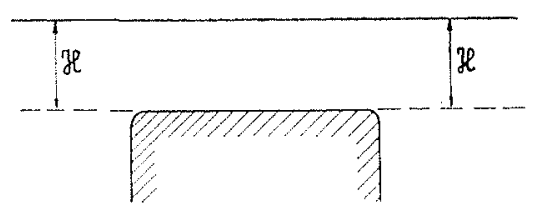

On a évidemment dans ce cas :

$$
\mathrm{M}=\frac{1}{\rho g l} \iint_{(1)} p d \sigma=\frac{\mathscr{H C}^{2}}{2}+\hat{\delta}=0
$$

lorsque $\mathrm{Q}=\mathrm{Q}_{m}$ nous avons vu que $\mathrm{M}=\left(2 \mathcal{Z} e^{\prime / 3}\right)$ d'où $\delta=\left(\not e^{2} / 6\right) ; \delta$ varic donc de 0 à $\not e^{2} / 6$ lorsque la hauteur d'eau amont $h$ sur le seuil varie de $\mathscr{H}$ à $2 \mathscr{H} / 3$.

Ces considérations ne sont valables que si le rayon de courbure du raccordement du seuil du déversoir avec sa paroi verticale d'amont est suffisant pour éviter tout décollement.
Nos équations supposent en effet que le radier du déversoir est une ligne de courant, ce qui n'est pas réalisé lor'squ'il y a apparition sur le seuil d'un point de décollement avec zone de dépression à l'aval.

Dans l'hypothèse d'un écoulement à potentiel des vitesses à l'amont du seuil, on a, en désignant par $\mathrm{V}$ la vitesse de l'eau en un point du parement amont :

$$
\delta=\frac{1}{l} \iint_{(2)} \frac{V^{2}}{2 g} d \sigma
$$

Nous poserons :

$$
\hat{o}=\frac{\mathrm{Q}^{2}}{2 g^{2} d} f\left(\frac{h}{d}\right)
$$

Pour un seuil amont bien arrondi, nos écuations ne nous permettent pas de déterminer $f\left(\frac{h}{\mathscr{H}}\right)$, mais elles nous donnent la valeur de cette fonction pour deux valeurs du rapport $\frac{h}{d \mathcal{C}}$

Pour :

$$
\frac{h}{\mathscr{H}}=\frac{2}{3} \quad 亏=\frac{\mathscr{H}^{2}}{6}=\frac{9}{16} \frac{\mathrm{Q}^{2}}{g l^{2} \mathscr{H C}}
$$

d'où :

$$
f\left(\frac{2}{3}\right)=\frac{9}{8}
$$

d'autre part, la pente de la tangente à la ligne d'eau sur le seuil est donnée d'après (18) par la relation :

$$
\begin{aligned}
\frac{\mathrm{Q}^{2}}{6 g l^{2}}\left(\frac{d y}{d x}\right)_{l=h}^{2} & =\mathscr{H} h^{2}-\frac{h^{3}}{2}+\frac{\mathrm{Q}^{2}}{2 g l^{2}} \\
& -\left[\frac{\mathcal{H}^{2} ?}{2}+\frac{\mathrm{Q}^{2}}{2 g l^{2} \mathscr{H}} f\left(\frac{h}{\mathscr{H}}\right)\right] h
\end{aligned}
$$

ll étant relié à Q par la relation :

$$
\mathscr{H}=h+\frac{\mathrm{Q}^{2}}{2 g l^{2} h^{2}}
$$

Lorsque le débit $\mathrm{Q}$ sur le déversoir tend vers 0 , $h$ tend vers 7 . La pente de la tangente à la ligne d'eau au point $\mathrm{B}(y=h)$ doit tendre vers 0 , ce qui impose $f(1)=1$.

Nous prendrons pour $f(h / J e)$ la fonction linéaire qui satisfait aux conditions précédentes. La comparaison des lignes d'eau obtenues expérimentalement avec celles que nous déduirons plus loin de nos équations, en tenant compte du frottement, montre que cette hypothèse est sensiblement conforme à la réalité.

Dans ces conditions, la valeur de la cons- 
Lante $\mathrm{M}$ pour un débit $Q$ el une hauteur d'eau 犯 amont a pour valeur :

$$
\mathrm{N}=\frac{\varkappa^{2}}{2}+\frac{\mathrm{Q}^{2}}{16 g^{2}}\left|11-\frac{3 h}{\pi}\right|
$$

DÉTERMINATION DE LA LIGNE D'LAU, THÉORIE DES ONDULATIONS DE SURFACE.

La ligne d'eau correspondant au débit $Q$ et a la charge amont Je est la courbe intégrale de l'équation différentielle $\left(18_{a}\right)$.

$$
\begin{aligned}
& \frac{\mathrm{Q}^{2}}{6 g^{2}}\left(\frac{d y}{d x}\right)^{2}=\mathcal{H} y^{2}-\frac{y^{3}}{2}+\frac{\mathrm{Q}^{2}}{2 g^{2}} \\
& -\left|\frac{\alpha^{2}}{2}+\frac{\mathrm{Q}^{2}}{8 g^{2} h}\left(1+\frac{3 h}{\pi}\right)\right| y\left(18_{n}\right)
\end{aligned}
$$

qui satisfait à la condition de CAUсHY $x=0 y=h$, $h$ étant la plus grande racine positive de l'équation :

$$
y=h+\frac{Q^{2}}{2 g l^{2}} h^{2}
$$

Pour discuter la forme de cette courbe, nous utiliserons les variables et coefficients sans dimensions.

$$
\begin{array}{ll}
\sigma=\frac{h}{\pi} & m=\frac{\mathrm{Q}}{l \mathscr{H} \sqrt{2 g} \mathscr{H}} \\
u=\frac{y}{\pi} & l=\frac{x}{\pi}
\end{array}
$$

Les équations précédentes s'écrivent :

$$
\begin{aligned}
\left(\frac{d u}{d t}\right)^{2} & \left.=\frac{3}{4 m^{2}}\right\rceil^{4} u^{2}-2 u^{3}+4 m^{2} \\
& \left.-\left|2+\frac{m^{2}}{2}(11-3 \sigma)\right| u\right\rangle \\
m^{2} & =\sigma^{2}(1-\sigma)
\end{aligned}
$$

La forme de la courbe dépend de la position de - par rapport aux racines du polynome du troisième degré :

$$
\begin{gathered}
\mathrm{F}(u)=-2 u^{3}+4 u^{2} \\
-\left\lceil 2+\frac{m^{2}}{2}(11-3 \sigma)\right\rceil u+4 m^{2}=0
\end{gathered}
$$

lorsque $m$ varie de 0 à $0,385=\frac{2}{3 \sqrt{3}}, \sigma$ décroìt de 1 à $2 / 3$, on a :

$$
\mathrm{F}(1)=-\frac{3 m^{2}}{2}(1-\sigma)^{2}<0
$$

$$
\begin{aligned}
& F(\sigma)=\frac{\sigma}{2}(2-\sigma)(1-\sigma) \div(3 \sigma-2)>0 \\
& F(0)=4 \sigma^{2}(1-\sigma)>0
\end{aligned}
$$

l'équation $\mathrm{F}(u)=0$ admet donc trois racines rélles positives pour $2 / 3<\sigma<1$ dont deux sont inférieures à o et la plus grande comprise entre $\sigma$ et 1 . Nous désignerons respectivement par $u_{0}=y_{0} / \mathscr{H} u_{1}=\left(y_{1} / \mathscr{H}\right)$ les deux racines qui encadrent $\sigma=(h / g e) \quad u_{0}<\sigma<u_{1}$.

Nous choisirons évidemment parmi les deux courbes intégrales de $\left(18_{t i}\right)$, qui passent par le point $t=0 \quad u=\sigma$, celle qui admet en ce point une pente mégative. Cette pente ayant pour valeur :

$\left(\frac{d u}{d L}\right)_{u=\sigma}=-\frac{1}{2} \sqrt{\frac{3(1-\sigma)(3 \sigma-2)(2-\sigma)}{2 \sigma}}$

Cette délermination étanl choisic, le polynome $F(u)$ devant rester positil, $u$ doit ètre compris entre les deux racines les plus élevées de F (u) : $u_{0}$ et $u_{1}$, la hauteur d'eau sur le seuil oscille done entre deux valeurs $y_{0}$ et $y_{1}$, pour une valeur du débit $Q$ comprise entre 0 et $Q_{m}$.

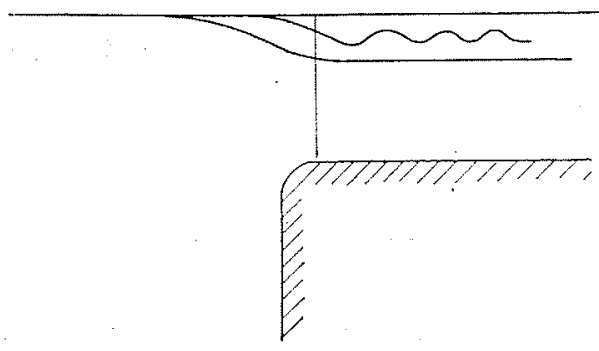

Fig. 41

Ces deux valcurs sont égales pour $Q=0 \mathrm{el}$ $Q=Q_{m}$.

En effet, pour $Q=0, F(u)$ admet une racine double $u=\sigma=1$.

$$
y_{0}=y_{1}=h=\not e
$$

Pour $Q=Q_{m}=\frac{2}{3 \sqrt{3}} l \not e \sqrt{2 g} \mathscr{H C}, \quad \mathrm{F}(u)$ admet une racine triple:

$$
u==\sigma=\frac{2}{3}, \quad y_{1}=y_{1}=h=\frac{2}{3} \mathrm{ce} ;
$$

dans les deux cas, la ligne d'eau sur le réservoir est une droite horizontale.

Nos équations mettent ainsi en évidence une forme classique de ligne d'eau comnue sous le nom de «nappe ondulée » et permettent de calculer l'amplitude des ondulations. La détermination de leur longueur d'onde se ramène, à l'aide de l'équation (18), au calcul d'une intégrale elliptique. Nous mettrons à cet effet l'érquation (18) sous une forme particulicre faisant intervenir les hauteurs $y_{0}$ et $y_{1}$. 
Considérons l'équation (18):

$$
\frac{\mathrm{Q}^{2}}{6 g l^{2}}\left(\frac{d y}{d x}\right)^{2}=\mathscr{H} y^{\prime \prime}-\frac{y^{*}}{2}+\frac{\mathrm{Q}^{2}}{2 g l^{*}} \mathrm{M} y
$$

en écrivant que $d y / d x=0$, pour $y==y_{0}$ et $y=y_{1}$, on obtient les relations :

$$
\begin{aligned}
& \mathscr{H}=\frac{y_{1}+y_{0}}{2}+\frac{\mathrm{Q}^{2}}{2 g l^{2} y_{1} y_{0}} \\
& \mathrm{M}=\frac{y_{1} y_{0}}{2}+\frac{\mathrm{Q}^{2}}{2 g l^{2} y_{1} y_{0}}\left(y_{1}+y_{0}\right)
\end{aligned}
$$

Reportant ces valeurs de $\mathcal{H}$ et $\mathrm{M}$ dans l'équation précédente, celle-ci se met sous la forme:

$\frac{\mathrm{Q}^{2}}{3 g l^{2}}\left(\frac{d y}{d x}\right)^{2}=\left(y-y_{0}\right)\left(y-y_{1}\right)\left(\frac{\mathrm{Q}^{2}}{g^{2} y_{1} y_{11}}-y\right)$

la demi-longueur d'onde $\mathrm{L}$ est donnée par l'intigrale :

$$
\mathrm{L}=\frac{\mathrm{Q}}{l \sqrt{3 g}} \int_{y_{0}}^{\mathrm{z} / 1} \frac{d y}{\sqrt{\left(y-y_{0}\right)\left(y-y_{1}\right)\left(\frac{\mathrm{Q}^{2}}{g l^{2} y_{0} y_{1}}-y\right)}}
$$

qui se transforme en une intégrale elliptique d'un type classique a l'aide du changement de variable :

$$
y=\frac{y_{1}+y_{0}}{2}+\frac{y_{1}-y_{0}}{2} u
$$

il vient :

$$
=\frac{\mathrm{Q}}{l \sqrt{3 g\left(\frac{y_{1}+y_{0}}{2}-\frac{\mathrm{Q}^{2}}{g l^{2} y_{1} y_{0}}\right)}} \int_{\mathrm{l}^{+1}}^{\frac{d u}{\sqrt{\left(1-u^{2}\right)(1+\mathrm{K} u)}}}
$$

avec :

$$
\mathrm{K}=\frac{y_{1}-y_{0}}{y_{1}+y_{0}-\frac{2 \mathrm{Q}^{2}}{g l^{2} y_{1} y_{0}}}
$$

intégrale elliptique uniformément convergente pour $-1<\mathrm{K}<1$.

$y_{1}, y_{0}$ et $\mathrm{Q}^{2} /\left(g l^{2} y_{0} y_{1}\right)$ sont les trois racines de léquation du troisième degré $\mathrm{F}(y / \mathcal{Z})=0$ classées par ordre décroissant:

$$
\frac{\mathrm{Q}^{2}}{g l^{2} y_{0} y_{1}}<y_{0}<y_{1}
$$

On a donc : $0<K<1$.

Dans ces conditions, la demi-Iongueur d'onde peut être calculée par un déreloppement en série uniformément convergent.

$$
\mathrm{I}_{\alpha}=\frac{\pi \mathrm{Q}}{l \sqrt{3 g\left(\frac{y_{1}+y_{0}}{2}-\frac{\mathrm{Q}^{2}}{g^{l^{2} y_{1} y_{0}}}\right)}}\left(1+\frac{3}{16} \mathrm{~K}^{2}+\cdots\right)
$$

Lorsque l'amplitude des ondulations $y_{1} \cdots y_{1}$ est faible, $K$ est petit, la ligne d'eau est sensiblement sinusoïdale et la demi-Iongueur d'onde est donnée sensiblement par le premier terme du développement.

DÉTERMINATION DE LA LIGNE D'EAU

LORSQU'ON TIENT COMPTE DU FROTTEMENT.

La ligne d'eau est alor's une solution $y=y(x)$ du système différentiel :

$$
\left\{\begin{array}{l}
\left.\frac{\mathrm{Q}^{2}}{6 g l^{2}} \frac{d}{d y}\right|^{-} \frac{1}{y}\left(\frac{d y}{d x}\right)^{2} \mid=\mathrm{H}-y \cdot \frac{\mathrm{Q}^{2}}{2 g l^{2} h^{2}} \\
\frac{d \mathrm{H}}{d x}=\cdots \frac{\mathrm{Q}^{2}}{c^{2} l^{2} y^{2}}
\end{array}\right.
$$

La charge $H$ et la fonction $M$ ne restent pas constantes, mais leurs valeurs dans la section amont, où la hauteur d'eau est $h$, ont des valeurs déterminées :

$$
\begin{gathered}
\mathrm{H}(h)=\mathscr{J} \\
\mathrm{M}(h)=\frac{\mathcal{H}^{2}}{2}+\frac{\mathrm{Q}^{2}}{16 g l^{2} \mathcal{H}}\left(11-\frac{3 h}{\not K}\right)
\end{gathered}
$$

La hauteur d'eau $h$ satisfaisant encore à l'équation:

$$
h+\frac{Q^{2}}{2 g l^{2} h^{2}}=\mathcal{H e}
$$

De $H(h)$ et $\mathrm{M}(h)$ on déduit la pente de la ligne d'eau sur le seuil :

$\frac{\mathrm{Q}^{2}}{6 g l^{2}}\left(\frac{d y}{d x}\right)_{y=n}^{2}=\mathrm{H}(h) h^{2}-\frac{h^{3}}{2}+\frac{\mathrm{Q}^{2}}{2 g l^{2}}-\mathrm{M}(h) h$

équation dont il faut prendre la racine négative.

La ligne d'eau est ainsi la courbe intégrale du système précédent qui satisfait aux conditions de CaUchy :

$$
\begin{gathered}
x=0 \quad y=h=\sigma \text { ze } \quad \mathrm{H}(h)=\text { Je } \\
\left(\frac{d y}{d x}\right)_{y=1}=-\frac{1}{2} \sqrt{\frac{3(1-\sigma)(3 \sigma-2)(2-\sigma)}{2 \sigma}}
\end{gathered}
$$

Pour une charge amont domnée Fe, les lignes d'cau correspondant aux différents débits $Q$ passant sur le seuil sont ainsi parfaitement déter minées, chacune d'elles correspondant à une condition aval particulière (par exemple la hauteur d'eau dans le réservoir aval).

La forme de la ligne de remous se dóduit de l'allure de la courbe (C) qui la représente dans le plan $\mathrm{HOy}$. 


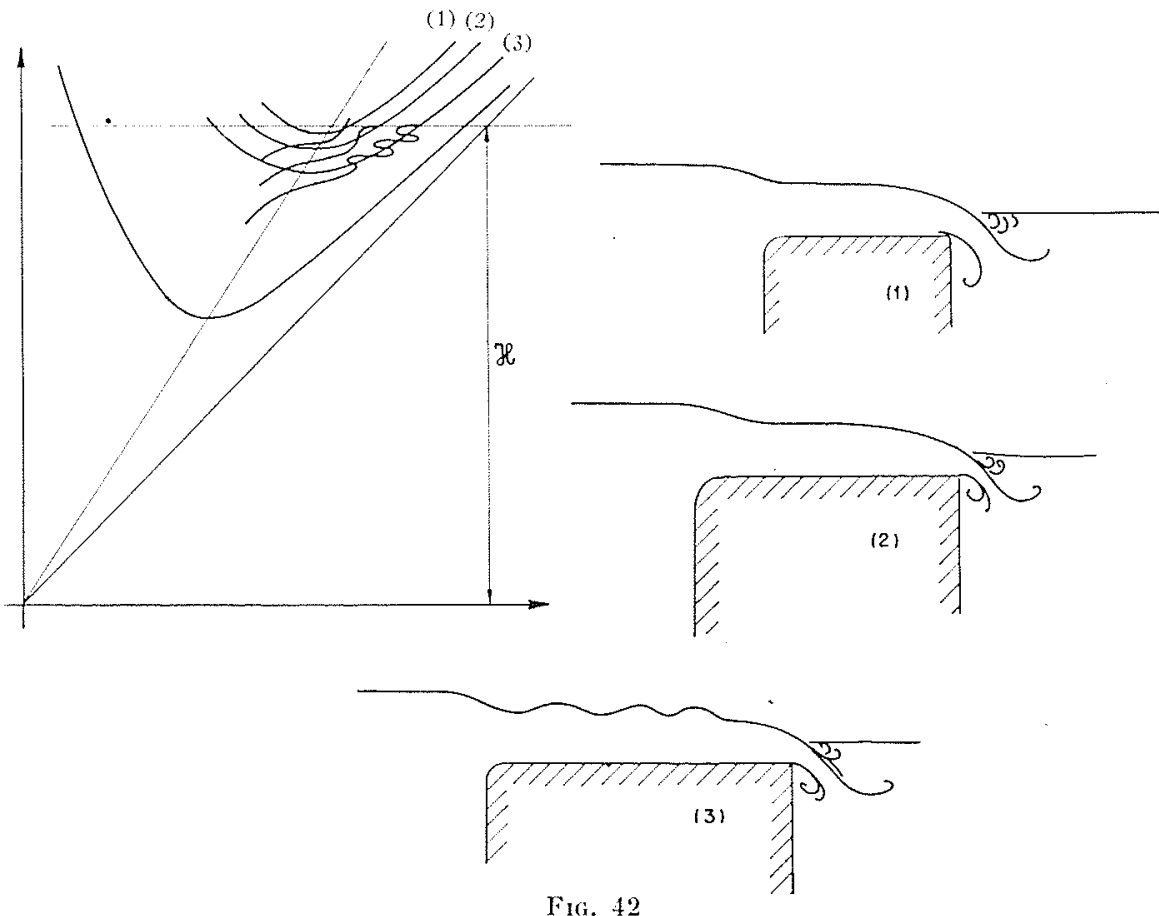

La figure 42 donne pour une charge amont $\mathscr{Z}$ le tracé des courbes $(C)$ correspondant à diverses valeurs du débit $Q$. La pente de la tangente a (C) au point $\mathrm{B} y=h=\sigma \mathscr{F}$ a pour valeur :

$$
\left(\frac{d \mathrm{H}}{d y}\right)_{y=h}=\frac{d \mathrm{H}}{d x} \cdot \frac{d x}{d y}=\frac{4 q}{c^{2}} \sqrt{\frac{2(1-\sigma)}{3 \sigma(3 \sigma-2)(2-\sigma)}}
$$

Il ne peut $y$ avoir de nappe ondulée que si la pente de la tangente en $B$ a la courbe $(C)$ est inférieure à la pente de la langente à la courbe $(H)$ d'équation $\mathrm{H}=y+\left(\mathrm{Q}^{2} / 2 g l^{2} y^{2}\right)$, c'est-à-dire si le débit $Q$ n'est pas trop voisin de $Q_{m}$.

La pente de la langente à la ligne de remous, $d y / d x$, al point d'ordonné $y$, est donne par l'équation intégro-différentielle :

$$
\begin{aligned}
\frac{\mathrm{Q}^{2}}{6 g l^{2}} \frac{1}{y}\left(\frac{d y}{d x}\right)^{2} & =\frac{\mathrm{Q}^{2}}{6 g l^{2}} \frac{1}{h}\left(\frac{d y}{d x}\right)_{y=h}^{2} \\
& +\int_{h}^{y /}\left(\mathrm{H}-y-\frac{\mathrm{Q}^{2}}{2 g l^{2} y^{2}}\right) d y
\end{aligned}
$$

La pente de la tangente à la ligne d'eau théorique, $(d y / d x)_{t}$, lorsqu'on néglige le frottement, serait donnée par l'équation :

$$
\begin{aligned}
& \frac{\mathrm{Q}^{2}}{6 g l^{2}} \frac{1}{y}\left(\frac{d y}{d x}\right)_{l}^{2}=\frac{\mathrm{Q}^{2}}{6 g l^{2}} \frac{1}{h}\left(\frac{d y}{d x}\right)_{y=n}^{2} \\
& +\int_{h}^{y}\left(\mathcal{H}-y-\frac{\mathrm{O}^{2}}{2 g l^{2} y^{2}}\right) d y
\end{aligned}
$$

Comme, par suite du frottement, $\mathbf{H}(y)$ est inférieur à $F e$, on en déduit que les points bas de la ligne de remous sont situés en dessous des points bas de la ligne d'eau théorique.
Lorsque le débit $Q$ est voisin de 0 , l'amplitude des ondulations et leur longueur d'onde diminuent considérablement, la courbe (C) est pratiquement confondue avec $(H)$, des phénomines de lension superficielle empechent la formation des ondulations.

Les lignes de remous correspondant à une charge amont $\mathscr{K}$, pour diverses valeurs de débil Q, sont représentées sur la figure 42 .

L'intégration des equations précédentes est impossible. Cependant, la détermination approchée de la ligne d'eau peut se faire, lorsque la nappe n'est pas ondulée, par une méthode aux différences finies à partir du systeme:

$$
\begin{aligned}
& \frac{\mathrm{Q}^{2}}{6 g l^{2}}\left(\frac{d ! y}{d x}\right)^{2}=\mathrm{H} y^{2}-\frac{y^{3}}{2}+\frac{\mathrm{Q}^{2}}{2 g l^{2}}-\mathrm{My} \\
& \frac{d \mathrm{H}}{d x}=-\frac{\mathrm{Q}^{2}}{c^{2} l^{2} y^{3}} \\
& \frac{d \mathrm{M}}{d x}==-\frac{\mathrm{Q}^{2}}{c^{2} l^{2} y^{2}}
\end{aligned}
$$

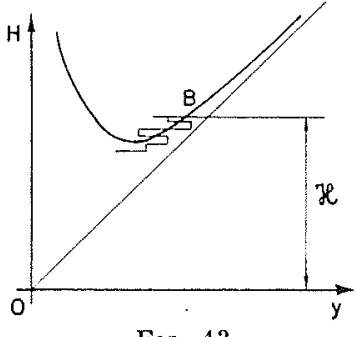

qui, on le démontre aisément, est équivalent aux systèmes (8) et $(9)$ 
La courbe de remous est définie par la solution du système précédent satisfaisant pour $x=0$ aux conditions de Cauchy :

$$
y=h \quad \mathrm{H}=\mathrm{H}(h)=\mathscr{H} \quad \mathrm{M}=\mathrm{M}(h)
$$

Dans le cas d'une nappe ondulée, il est préfirable d'utiliser une autre méthode d'intégration approchée, basée sur la remarque suivante : entre deux sommets de la courbe de remous, la charge $H$ varie extrêmement peu, on peut done la considérer comme constante. Cela revient ì remplacer la courbe (C) du plan HOy par une ligne en escaliers formée de segments de droites (voir fig. 43). D'après les résultats du paragraphe précédent, les abscisses $y_{0 n}, y_{1 n}$ des extrimités d'un segment horizontal d'ordonnée $H_{n}$ sont liées par la relation :

$$
\mathrm{H}_{n}=\frac{y_{(m, n}+y_{1 n}}{2}+\frac{\mathrm{Q}^{2}}{2 g^{\prime \prime} y_{m n} y_{\mathrm{i} n}}
$$

relation dont l’interprétation gémetrique a dejà élè précisée.

Les aires hachurées sur la figure 44 sont égales.

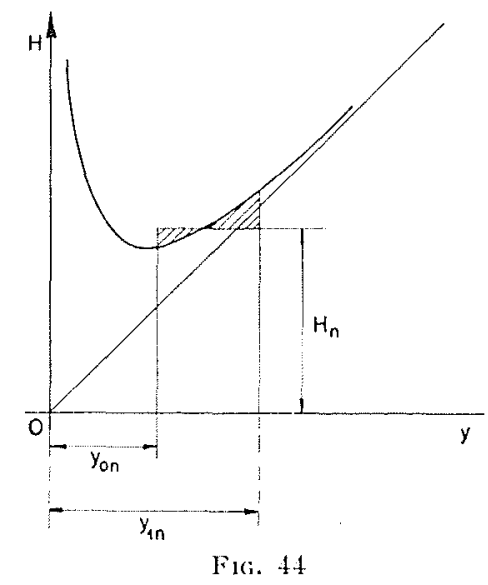

Le premier point bas de la ligne d'eau, correspondant à la hauteur d'eau $y_{01}$, se détermine à l'aide des considérations exposées au paragraphe précédent. $y_{01}$ est la racine moyenne de l'équation du troisième degré $\mathrm{F}(y / \mathcal{F})=0$. L'abscisse $x_{0}$ du point correspondant de la ligne d'eau est donnée par une intégrale elliptique.

La charge $\mathrm{H}_{0}$ dans cette section se déduit par un intégration aux différences finies de la relation différentielle:

$$
\frac{d \mathrm{H}}{d x}=-\frac{\mathrm{Q}^{2}}{c^{2} l^{2} y^{3}}
$$

lorsque $x$ varie de 0 à $x_{0}$, la valeur de $H$ pour $x=0$ étant $\mathscr{H}$.

La hauteur $y_{11}$ du premier point haut de la courbe de remous se déduit de la relation (21) ou l'on fait $y_{0 n}=y_{01}$ et $\mathrm{H}_{n}=\mathrm{H}_{0}$.
On obtient une équation du second degré en $y_{11}$ dont il convient de prendre la racine la plus élevée. L'abscisse de ce point est $x_{0}+\mathrm{L}_{0}, \mathrm{~L}_{0}$ étant donné par l’intégrale elliptique:

$$
\mathrm{L}_{0}=\frac{\int^{+1} \frac{d u}{\sqrt{\left(1-l^{2}\right)(1+\mathrm{K} u)}}}{l \sqrt{3 g\left(\frac{y_{01}+y_{11}}{2}-\frac{\mathrm{Q}^{2}}{g l^{2} y_{01} y_{11}}\right)}}
$$

On détermine ainsi de proche en proche les abscisses et les ordonnées des différents sommets de la ligne d'eau. L'examen de la figure 43 montre que ces ordonnées vont en diminuant et que les ondulations disparaissent lorsque la hauteur d'eau se rapproche de la hauteur critique. La ligne de remous peut alors se déterminer par une intégration aux différences finies du système (20); on voit sur la figure 43 que la courbe (C) est située au-dessous de $(\mathbf{H})$ et que l'aire comprise entre ces deux courbes croît rapidement lorsque $y$ décroît. $(d y / d x)^{2}$ auggmente donc, et comme la pente $d y / d x$ est négative, elle décroît lorsque $x$ augmente. La hauteur d'eau dans une section diminue donc très vite à mesure que cette section se rapproche de l'extrémité aval du seuil.

Il reste à examiner les conditions limites auxquelles doit satisfaire la ligne d'eau à l'extrémité.

\section{Conditions aUX Limites a L'EXTRÉmité AVAT.}

Nous supposerons que le déversoir communique à l'aval avec un réservoir de grande capacité où le niveau du plan d'eau peut occuper une position quelconque par rapport au seuil.

Nous désignerons éventuellement par $h_{a}$ la cote du plan d'eau dans le réservoir au-dessus du radier du déversoir et par $y_{a}$ la hauteur d'eau à l'extrémité aval du seuil. Lorsque $h_{a}$ est négatif (fig. 45), la pression atmosphérique régne sous la face inférieure de la nappe lorsque l'air a librement accès sous celle-ci (écoulement en

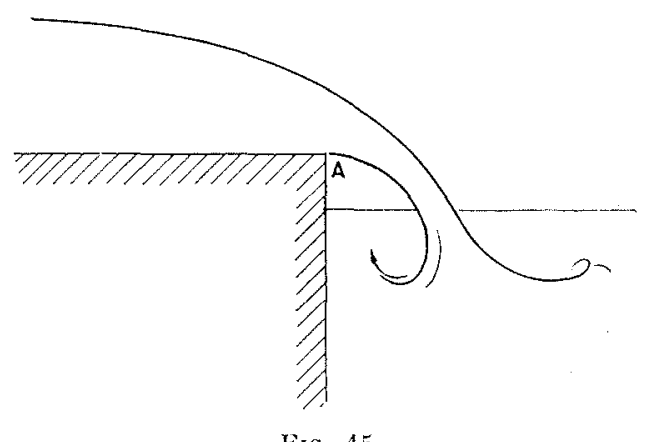

Fic. 45 


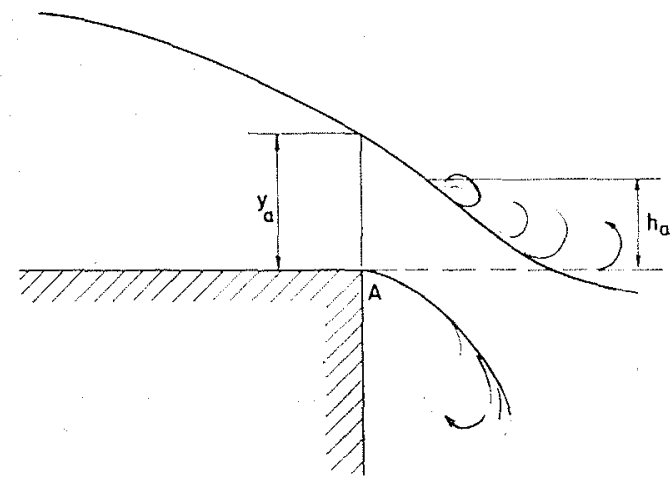

Fic. 46

nappe libre). Il faut donc écrire que Ia pression est nulle au point A de la section extrême.

Lorsque $h_{a}$ est positif, A est toujours point de décollement et il existe directement à l'aval du déversoir une zone de fluide mort qui ne participe pas à l'écoulement. Il faudra done écrire que la pression au point $\mathrm{A}$ est égale à $h_{a}$.

En se reportant à l'équation (5), qui donne la pression en un point d'une section en fonction de la cote de ce point au-dessus du radier, on est conduit à la relation (dans l'hypothèse d'une répartition uniforme des vitesses normales) :

$$
h_{a}=y_{a}+\frac{\mathrm{Q}^{2}}{2 g^{2} y_{a}}\left[\frac{d^{2} y}{d x^{2}}-\frac{1}{y_{a}}\left(\frac{d y}{d x}\right)^{2}\right]_{y=y / u}
$$

La comparaison avec l'équation qui définit la fonction $M(y)$ :

$$
\begin{aligned}
\mathrm{M}\left(\boldsymbol{y}_{a}\right)= & \frac{y_{a}^{2}}{2}+\frac{\mathrm{Q}^{2}}{g^{L^{2}} y_{a}} \\
& +\frac{\mathrm{Q}^{2}}{3 g l^{2} y_{a}}\left[\frac{d^{2} y}{d x^{2}}-\frac{1}{y_{a}}\left(\frac{d y}{d x}\right)^{2}\right]_{y=y_{a}}
\end{aligned}
$$

conduit à une relation entre la hauteur d'eau $y_{a}$ et la fonction $M\left(y_{0}\right)$ dans la section aval du seuil :

$$
\mathrm{M}\left(y_{a}\right)=\frac{\mathrm{Q}^{2}}{g^{l^{2}} y_{a}}-\frac{y_{a}^{2}}{6}+\frac{2}{3} h_{a} y_{a}
$$

Cette relation, valable lorsque $h_{a}>0$, constitue la condition limite aval. Dans l'hypothèse d'un écoulement en nappe libre, il convient évidemment de faire $h_{\alpha}=0$.

Il est donc possible de déterminer la hauteur d'eau $h_{a}$ dans le réservoir qui correspond au passage d'un débit $Q$ sur le déversoir et la hauteur d'eau $y_{a}$ à l'extrémité du seuil.

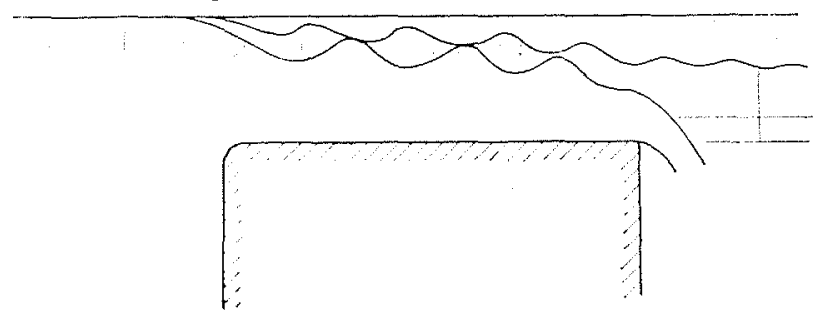

Fix. 48

\section{COMPARAISON ENTRE L'ÉTUDE THÉORIQUE D'UNE NAPPE ONDULEEE SUR UN DÉVERSOIR A SEUIL EPAIS ET LES RÉSULTATS OBTENUS EXPÉRIMENTALEMENT}

Ces essais, entrepris au laboratoire par M. Tison, professeur à l'Université de Gand, concernent, en particulier, les caractéristiques (amplitude, longueur d'onde) des nappes ondulées obtenues sur un déversoir à seuil épais pour diverses charges amont.

\section{CARACTERISTIQUES DU DÉVERSOIR, RÉSULTAT DES ESSAIS.}

largeur $I=0,50 \mathrm{~m}$, longueur $1,80 \mathrm{~m}$, pente $i=0$ plan d'eau aval situé au-dessous du seuil (écoulement en nappe libre).

Le rayon de courbure du raccordement du seuil avec la paroi verticale d'amont est suffisant pour éviter tout décollement sous les charges envisagées.

Le seuil est rendu rugueux par cimentage avec sable très rude, il correspond à un coefficient de BAZIN $\gamma=0,41$.

Les débits et les longueurs d'ondes des ondulations obtenues pour diverses charges amont, comptées à partir du seuil déversant, sont précisés dans le tableau ci-après.

TABLEAU I

\begin{tabular}{|c|c|c|}
\hline $\begin{array}{c}\text { Charge amont } \\
\mathcal{L} \\
\text { (mètres) }\end{array}$ & $\begin{array}{c}\text { Débit } \\
\mathrm{Q} \\
(\mathrm{m} 3 / \mathrm{s})\end{array}$ & $\begin{array}{c}\text { Longueur d'onde } \\
2 \mathrm{~L} \\
\text { (mètres) }\end{array}$ \\
\hline 0,0672 & 0,0115 & 0,135 \\
\hline 0,0875 & 0,0180 & 0,200 \\
\hline 0,1210 & 0,0308 & 0,300 \\
\hline 0,1400 & 0,0396 & 0,365 \\
\hline
\end{tabular}


La figure I donne pour le même déversoir et la charge amont $\chi^{\circ}=0,1400$, le trace de la ligne d'eau déterminée expérimentalement au laboratoire (en trait plein); la ligne en traits mixtes est celle obtenue à partir de la théorie des écoulements graduellement variés, la hauteur critique étant atteinte à l'extrémité aval du seuil.

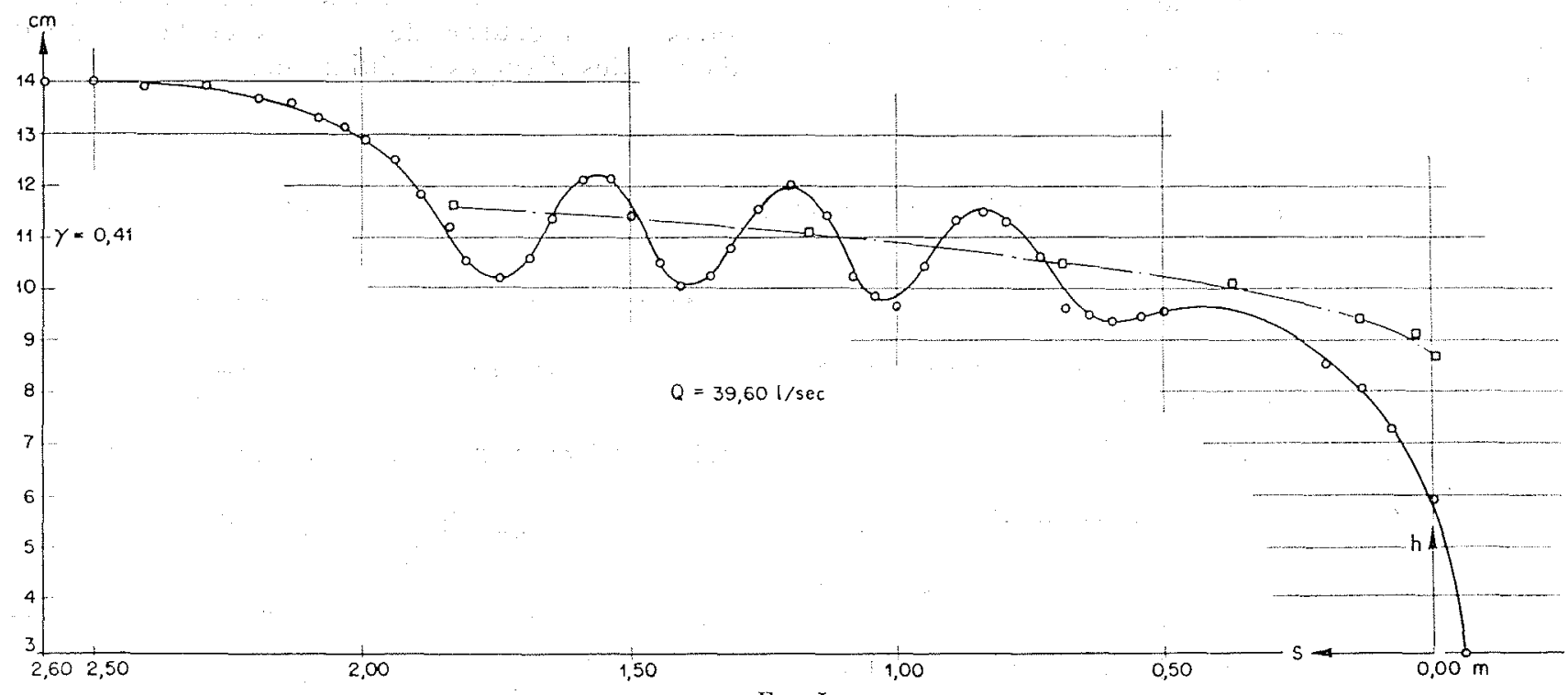

FIG. I

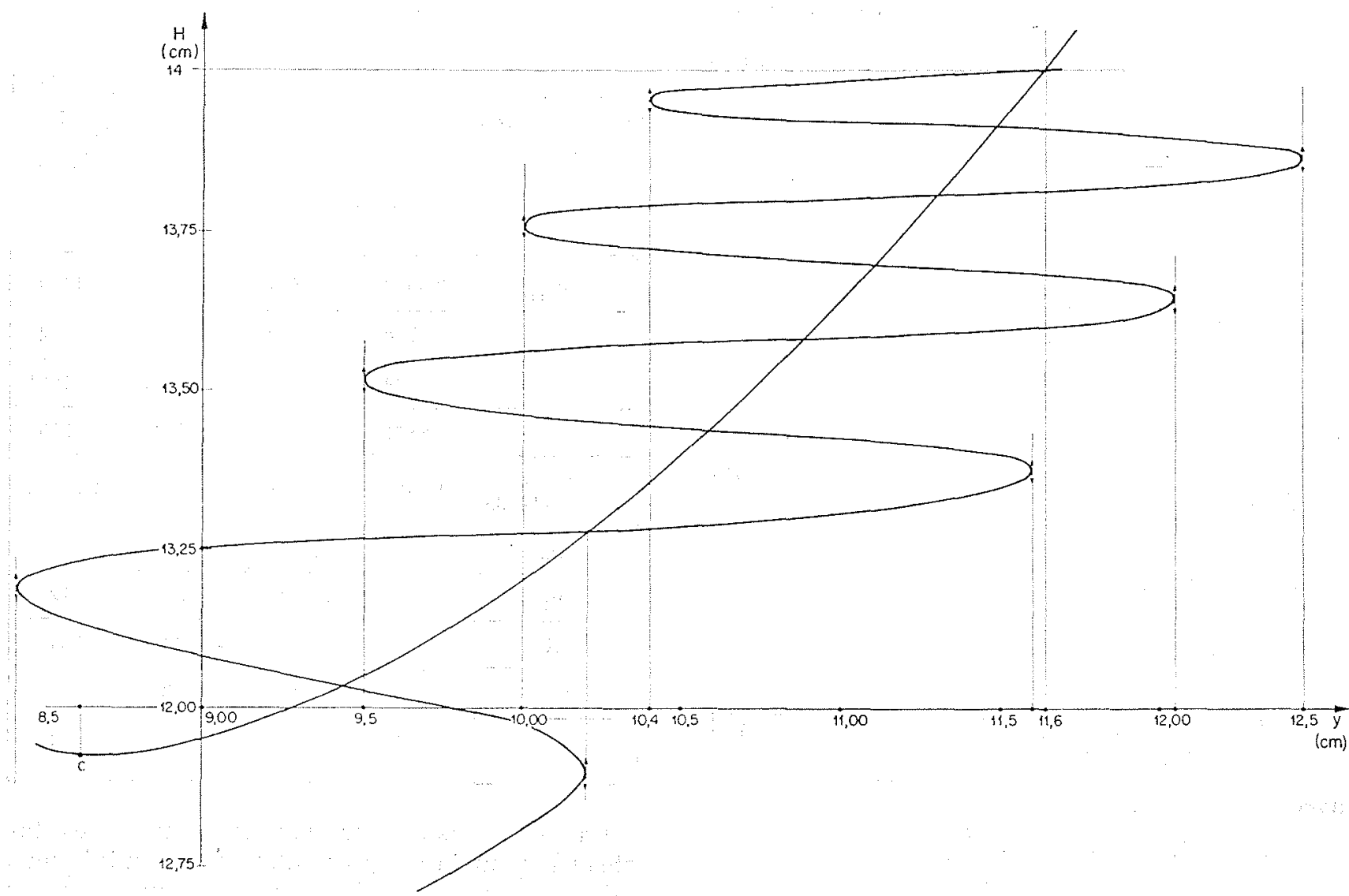

Frg. II 
RÉsultats ThÉoriques DÉduit's DES ÉUATIONS PRÉCÉDENTES.

Nous avons calcule, pour les diverses valeurs mesurées de la charge Je et du débit Q, l'ampliture et la longueur d'onde thérique des ondulations, en supposant la charge constante sur le déversoir et égale à la charge amont $\mathcal{H e}$.
Dans l'hypothèse d'une charge amont :

$$
\text { He }=0,1400 \mathrm{~m}
$$

correspondant au débit $Q=0,0396 \mathrm{~m}^{3} / \mathrm{s}$, nous avons déterminé la ligne d'eau à partir de nos équations en tenant compte des pertes de charge et calculé en particulier les ordonnées des sommets de la courbe de remous et la longueur d'onde des diverses ondulations.

\section{1. - Caractéristiques des ondulations en supposant la charge constante sur le déversoir.}

\section{Notations}

$h=$ hauteur d'eau sur le seuil amont du déversoir

ye = charge amont;

$q=\frac{Q}{l}=$ débit par mètre linéaire;

$y_{0}=$ ordonnée du point bas de la ligne d'eall;

$y_{1}=$ ordonnée du point haut de la ligne d'eau;

$a=y_{1}-y_{0}=$ amplitude des ondulations;

$2 \mathrm{~L}=$ longueur d'onde des ondulations.

Nous utiliserons pour les calculs les variables sams dimensions suivantes:

$$
\begin{aligned}
\sigma=\frac{h}{\mathscr{H}} & m=\frac{q}{\mathscr{H} \sqrt{2 g \mathscr{H}}} \\
u_{0}=\frac{y_{0}}{\mathscr{H}} & u_{1}=\frac{y_{1}}{\mathscr{H}}
\end{aligned}
$$

o est la racine de l'équation :

$$
\sigma^{2}(1-\sigma)=m^{2}
$$

qui correspond au régime fluvial.

$u_{0}$ et $u_{1}$, les deux racines les plus élevées de l'équation du troisième degré :

$-u^{3}+2 u^{2}-\left[1+\frac{m^{2}}{4}(11-3 \sigma)\right] u+2 m^{2}=0$

La longueur d'onde est donnée par la relation :

$2 \mathrm{~L}=\frac{2 \pi q}{\sqrt{3 g\left(\frac{y_{1}+y_{0}}{2}-\frac{q^{2}}{g y_{1} y_{0}}\right)}}\left[1+\frac{3 \mathrm{~K}^{2}}{16}\right]$ avec :

$$
\mathrm{K}=\frac{y_{1}-y_{0}}{y_{1}+y_{0}-\frac{2 q^{2}}{g y_{1} y_{0}}}
$$

En tenant compte de la relation :

$$
\mathscr{H}=\frac{y_{1}+y_{0}}{2}+\frac{q^{2}}{2 g y_{1} y_{0}}
$$

qui s'écrit en variables sans dimensions :

$$
1=\frac{u_{1}+u_{0}}{2}+\frac{m^{2}}{u_{0} u_{1}}
$$

les valeurs de $\mathrm{L}$ et $\mathrm{K}$ sont données par les expressions :

$$
\begin{gathered}
\mathrm{K}=\frac{u_{1}-u_{0}}{3\left(u_{1}+u_{0}\right)-4} \\
2 \mathrm{~L}=\frac{4 \pi \mathcal{J} m}{\sqrt{9\left(u_{1}+u_{0}\right)-12}}
\end{gathered}
$$

Les valeurs de a et $2 \mathrm{~L}$ obtenues par le calcul a partir des données mesurées fe et $Q$ du tableau I sont précisées dans le tableau II.

\section{TABLEAU II}

\begin{tabular}{|c|c|c|c|c|}
\hline $\mathcal{F}_{(\mathrm{m})}$ & 0,0672 & 0,0875 & 0,1210 & 0,1400 \\
\hline$q=Q / l_{\left(\mathrm{m}^{3} / \mathrm{s}\right)}$ & 0,0230 & 0,0360 & 0,0616 & 0,0792 \\
\hline$m^{2}$ & 0,08885 & 0,09860 & 0,10917 & 0,11651 \\
\hline$\sigma$ & 0,8871 & 0,8696 & 0,8484 & 0,8315 \\
\hline $\begin{array}{c}1+\left(\mathrm{m}^{2} / 4\right) \\
(11-3 \sigma)\end{array}$ & 1,1852 & 1,20685 & 1,23075 & 1,24775 \\
\hline$u_{0}$ & 0,830 & 0,804 & 0,771 & 0,745 \\
\hline$u_{1}$ & 0,943 & 0,933 & 0,922 & 0,912 \\
\hline $\mathrm{K}$ & 0,086 & 0,107 & 0,280 & 0,354 \\
\hline$a_{(\mathrm{m})}$ & 0,008 & 0,011 & 0,018 & 0,023 \\
\hline $2 \mathrm{~L}_{(\mathrm{m})}$ & 0,127 & 0,182 & 0,280 & 0,354 \\
\hline
\end{tabular}

La comparaison avec les valeurs mesurées (tableau I) pour la longueur d'onde des ondulations fait apparaître une différence systématique par 
défaut en ce qui concerne les longueurs d'onde calculées, inférieures de 4 à $9 \%$ à celles mesurées. On obtiendrait une meilleure approxima- tion, en tenant compte des pertes de charge sur le déversoir, comme le montre l'étude de la ligne d'eau pour une charge amont $\mathscr{F}=0,14 \mathrm{~m}$.

\section{2. - Détermination théorique de la ligne d'eau dans l'hypothèse d'une charge amont $\mathscr{H}=0,14 \mathrm{~m}$}

La courbe de remous $y=y(x)$ et la charge sur le déversoir $H=H(x)$ sont les solutions du systène différentiel :

$$
\left\{\begin{array}{l}
\frac{q^{2}}{6 g} \frac{d}{d y}\left[\frac{1}{y}\left(\frac{d y}{d x}\right)^{2}\right]=\mathrm{H}-y-\frac{q^{2}}{2 g y^{2}} \\
\frac{d \mathrm{H}}{d x}=-\frac{q^{2}}{c^{2} y^{2} \mathrm{R}}=-\psi(y)
\end{array}\right.
$$

avec les notations suivantes:

$q=\frac{Q}{l}$ débit au mètre linéaire

$$
=\frac{0,0396}{0,50}=0,0792 \mathrm{~m}^{3} / \mathrm{s}
$$

$\mathrm{R}=$ Rayon hydraulique $=\frac{l y}{l+2 y} \quad(l=0,50)$

$c=$ Coefficient de Chézy donné par la formule de BAZIN avec $\gamma=0,41$

$$
c=\frac{87}{1+\frac{\gamma}{\sqrt{\mathrm{R}}}} \quad \psi(y)=\frac{q^{2}}{c^{2} y^{2} \mathrm{R}}
$$

II faut rechercher la solution du système qui satisfait à l'entrée du déversoir $(x=0)$ aux conditions de CAUCHY suivantes :

On connaît, pour $x=0$,

la charge :

$$
\mathrm{H}=\mathscr{H}=0,1400
$$

Ia hauteur d'eau :

$$
h=\sigma \mathscr{H}=0,831 \times 0,1400=0,116
$$

la fonction d'impulsion :

$$
\begin{aligned}
\mathrm{M}(h) & =\underset{2}{\mathcal{H}^{2}}\left[1+\frac{m^{2}}{4}(11-3 \sigma)\right] \\
& =\frac{1}{2} \times(0,14)^{2} \times 1,24775=0,01223
\end{aligned}
$$

ce qui revient, nous l'avons vu, à se donner la pente de la tangente à la ligne d'eau $\left(\begin{array}{c}d y \\ d x\end{array}\right)_{y=h}$ sur le seuil déversant.

La solution correspondante est représentée dans le plan HOy par une courbe (C) dont les sommets parallèles à $O H$ sont situés de part et d'autre de la courbe $(\mathrm{H})$ d'équation $\mathrm{H}=y+q^{2} /\left(2 g y^{2}\right)$ représentée figure II.

Soient $y_{0}$ et $y_{1}$ les ordonnées de deux sommets consécutifs de (C) correspondant respectivement à un point bas et un point haut de la ligne d'eau. En intégrant la première équation, compte tenu de $(d y / d x)=0$ pour $y=y_{0}$, on obtient :

$$
\begin{aligned}
\frac{q^{2}}{6 g}\left(\frac{d y}{d x}\right)^{2}=\int_{y_{0}}^{y} \mathrm{H} d y-\frac{y^{2}}{2} & +\frac{q^{2}}{2 g y} \\
& +\frac{y_{0}^{2}}{2}-\frac{q^{2}}{2 g y_{0}}
\end{aligned}
$$

Soit $\mathbf{H}_{m}$ la valeur moyenne de la charge dans l'intervalle $y_{0} y_{1}$, définie par l'expression :

$$
\mathbf{H}_{m}=\frac{1}{y_{1}-y_{0}} \int_{y_{0}}^{y_{1}} \mathrm{H} d y
$$

en écrivant que $(d y / d x)=0$, pour $y=y_{1}$, on obtient une relation entre les ordonnées de deux sommets consécutifs de la ligne d'eau et la charge moyenne dans l'intervalle:

$$
\mathrm{H}_{m}=\frac{y_{0}+y_{1}}{2}+\frac{q^{2}}{2 g y_{0} y_{1}}
$$

L'ordonnée $y_{00}$ du premier point bas à partir des considérations exposées au paragraphe I:

$$
y_{00}=u_{0} \mathcal{F}=0,745 \times 0,14=0,104
$$

son abscisse est sensiblement égale au quart de la longueur d'onde théorique, soit $\mathrm{L} / 2=0,090$.

La variation de la charge entre l'entrée du déversoir et le premier point bas s'obtient à partir de la deuxième équation :

en posant

$$
\frac{d \mathrm{H}}{d x}=-\psi(y)
$$

$$
\Delta \mathrm{H}=-\frac{1}{4} \mathrm{~L}\left[\psi(h)+\psi\left(y_{00}\right)\right]
$$

(voir tableau. III) 
La détermination de l'abscisse et de l'ordonnée $y_{10}$ du premier point haut, ainsi que la charge en ce point se fait par une méthode d'approximations successives à partir de la relation $(R)$.

On se donne une valeur de la charge moyenne, entre les deux premiers sommets consécutifs, $\mathbf{H}^{\prime}{ }_{m}$ légèrement inférieure à la charge $\mathrm{H}_{00}$ au point d'ordonnée $y_{00}$. On obtient de la sorte une première valeur approchée $y_{10}^{\prime}$ de l'ordonnée $d u$ premier point haut qui est la plus grande racine de l'équation :

$$
\mathrm{H}_{m}^{\prime}=\frac{y_{00}+y}{2}+\frac{q^{2}}{2 g y_{00} y}
$$

On en déduit une première valeur de la demilongueur d'onde :

$$
L^{\prime}=\frac{\pi q}{\sqrt{\frac{3 g\left(y_{00}+y_{10}^{\prime}\right)}{2}-\frac{q^{2}}{g y_{00} y_{10}^{\prime}}}}\left[1+\frac{3 \mathrm{~K}^{2}}{16}+\ldots\right]
$$

et une variation de la charge outre les deux points, donnée, à partir de l'équation 2, par l'expression :

$$
\Delta \mathrm{H}^{\prime}=-\frac{1}{2} \mathrm{~L}^{\prime}\left[\psi\left(y_{00}\right)+\psi\left(y^{\prime}{ }_{10}\right)\right]
$$

d'où l'on tire une valeur $\mathrm{H}^{\prime \prime}{ }_{m}=\mathrm{H}_{00}-\left(\Delta \mathrm{H}^{\prime} / 2\right)$ de la charge moyenne entre les deux sommets. Si cette valeur ne coïncide pas avec $\mathrm{H}^{\prime}$, on recommence le calcul précédent en partant de la nouvelle valeur $\mathrm{H}^{\prime \prime}{ }_{m}$. La convergence est rapide.

Les résultats définitifs sont représentés sur le

\begin{tabular}{|c|c|c|c|c|c|}
\hline$y$ & $\Psi(y)$ & $L$ & $\Delta H$ & $H(y)$ & $\mathrm{H}_{m}$ \\
\hline$h=0,116$ & 0,00469 & & & 0,1400 & \\
\hline$y_{00}=0,104$ & 0,00655 & 0,090 & $-0,00051$ & 0,13949 & \\
\hline & 0.00373 & 0,181 & $-0,00093$ & & 0,1390 \\
\hline$g_{10}-0,160$ & (0,000, & 0,186 & $-0,00104$ & 0,158000 & 0,1380 \\
\hline$y_{01}=0,100$ & 0,00745 & 0,192 & -0.00111 & 0,13752 & 0.1370 \\
\hline$y_{11}=0,121$ & 0,00412 & & & 0,13641 & \\
\hline$y_{02}=0,095$ & 0,00872 & 0,200 & $-0,00128$ & 0,13513 & 0,1358 \\
\hline$y_{12}=0,116$ & 0,00469 & 0,211 & $-0,00141$ & 0,13372 & 0,1344 \\
\hline$y_{03}=0,084$ & 0,00984 & 0,250 & $-0,00182$ & 0,13190 & 0,1328 \\
\hline$y_{13}=0,102$ & 0,00700 & 0,344 & $-0,00290$ & & 0,1304 \\
\hline
\end{tabular}
tableau ci-après :

\section{Tableau III}

La comparaison avec les valeurs mesurées pour les ordonnées des sommets fait apparaitre une bonne concordance avec les valeurs dédui- tes du calcul, sauf en ce qui concerne la dernière ondulation (voir tableau IV), la méthode approchée utilisée, qui consiste à utiliser la valeur moyenne de la charge, conduisant à une valeur trop élevée pour la longueur d'onde de l'ondulation.

\section{TABLEAU IV}

Valeur mesurée...|0,104|0,122 $|0,100| 0,120|0,097| 0,116|0,094| 0,097$ Valeur calculée... $|0,104| 0,125|0,100| 0,121|0,095| 0,116|0,084| 0,102$

La longueur d'onde calculée pour la première ondulation déduite du tableau III :

$$
2 \mathrm{~L}=0,181+0,186=0,368
$$

donne une valeur peu différente de celle mesurée 0,365 .

La courbe figurative (C) de la solution du système différentiel dans le plan $\mathrm{HO} y$ est représentée sur la figure II, sur laquelle a été tracée également la courbe $(\mathbf{H})$ d'équation :

$$
\mathrm{H}=y+\frac{q^{2}}{2 g y^{2}}
$$

qui passe par un minimum :

$$
\mathbf{H}_{r}=\frac{3}{2} \sqrt[3]{\frac{q^{2}}{g}}=0,1292
$$

pour la hauteur critique:

$$
y_{c}=\sqrt[3]{\frac{q^{2}}{g}}=0,0861
$$

L'étude théorique du système montre que les ondulations ne peuvent exister lorsque la charge devient inférieure à la charge critique $\mathrm{H}_{c}=0,1292$. Cette éventualité se produit dans la partic aval du déversoir; la quantité $\mathrm{H}-y-\left(q^{2} / 2 g y^{2}\right)$ devient négative et va en augmentant en valeur absolue. La courbe (C) est alors située au-dessous de $(\mathbf{H})$ et l'effet de courbure prend une valeur particulière.

L'expérience confirme ce résultat théorique; elle montre en effet que la théorie des « écoulements graduellement variés »n'est pas applicable à la portion aval du déversoir. Dans la portion amont, cette théorie permet de préciser la hauteur moyenne de la ligne d'eau sans donner aucun renseignement sur la longueur d'onde et l'amplitude des ondulations.

La théorie de seconde approximation donne des résultats plus conformes à l'expérience. Elle permet de montrer qu'à l'extrémité aval du déversoir, la hauteur d'eau et la charge sont, pour un écoulement en nappe libre, inférieurs à la 
hauteur et à la charge critique. Elle permet de préciser le nombre, la longueur d'onde et l'amplitude des ondulations de la surface libre.

La longueur d'onde est fonction de la charge amont et du coefficient de débit; l'amplitude dépend de la valeur que prend la fonction d'impulsion sur le seuil déversant, c'est-à-dire de la poussée sur la paroi amont du déversoir.

\section{V. - ETUDE DES ECOULEMENTS EN RÉGIME VARIABLE}

\section{1. - Equations aux dérivées partielles des écoulements variables.}

Nous nous proposons d'établir les équations aux dérivées partielles qui régissent la ligne d'eau en mourement variable dans l'hypothèse d'un canal prismatique de section rectangulaire, de faible pente et de grande largeur.

Comme dans l'étude des écoulements permanents, nous étudierons le mouvement du fluide par rapport à des axes rectangulaires $O x$ dirigé vers le bas suivant l'axe du radier, $O z$ perpendiculaire au radier dirigé vers le haut.

Nous adopterons les notations suivantes: $y(x, t)$ hauteur d'eau dans la section d'abscisse $x ; u(z, x, t), w(z, x, t)$ composantes sur les axes $\mathrm{O} x$ et $\mathrm{O} z$ de la vitesse du fluide au point de cote $z$ de la section d'abscisse $x$.

$q(x, t)=\mathrm{Q} / l$ débit par unité de largeur dans la section d'abscisse $x$ :

$$
\begin{gathered}
q=\int_{0}^{y} u d z \\
\mathrm{U}(x, t)=\frac{q}{y}=\frac{1}{y} \int_{0}^{y} u d z
\end{gathered}
$$

vitesse moyenne dans la section d'abscisse $x$.

La surface libre du fluide n'est plus ici une ligne de courant, mais une trajectoire. Sur cette surface limite mobile $u, w$ et $y$ ne sont donc pas indépendants et doivent satisfaire à la condition:

$$
w(y, x, t)=u(y, x, t) \frac{\partial y}{\partial x}+\frac{\partial y}{\partial t}
$$

$u$ et $w$ satisfont à l'équation de continuité.

$$
\frac{\partial u}{\partial x}+\frac{\partial w}{\partial z}=0
$$

qui exprime que l’intégrale curviligne :

$$
\int_{(\mathrm{C})} \bar{V} \cdot \bar{n} d s
$$

étendue à un contour (C) quelconque tracé dans le fluide est nulle. Cette condition doit donc s'appliquer au contour particulier représenté sur la figure 49.
Considérons la portion de fluide comprise entre les deux sections normales infiniment voisines d'abscisse $x$ et $x+d x$, le radier et la

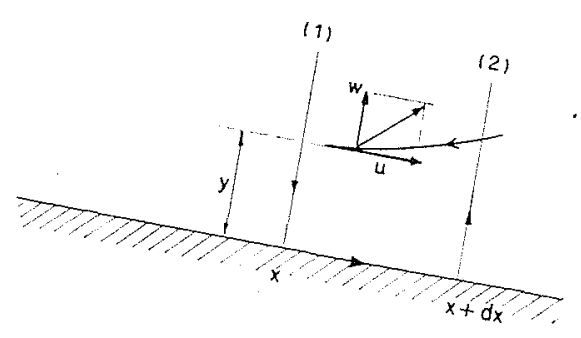

Fig. 49

surface libre. Ces deux sections limitent sur la surface libre un élément d'arc $d s$.

La vitesse du fluide à la surface et la normale extérieure sont définies par les vecteurs:

$$
\begin{gathered}
\bar{v}=u(y, x, t) \bar{x}+w(y, x, t) \bar{z} \\
\bar{n}=\frac{d x}{d s}\left[-\frac{d y}{d x} \bar{x}+\bar{z}\right]
\end{gathered}
$$

l'intégrale curviligne $\int_{(C)} \bar{V} \cdot \bar{n} d s$ étendue à ce contour s'écrit donc :

$q(x+d x, t)-q(x, t)+$

$$
\left[-u(y, x, t) \frac{d y}{d x}+u(y, x, t)\right] d x=0
$$

c'est-à-dire :

$$
\frac{\partial q}{\partial x}+w(y, x, t)-u(y, x, t) \frac{\partial y}{\partial x}=0
$$

ou en tenant compte de (20):

$$
\frac{\partial q}{\partial x}+\frac{\partial y}{\partial t}=0
$$

Appliquons maintenant le théorème des quantités de mouvement à la portion de fluide com- 
prise entre les sections (1) et (2). En projection sur l'axe $\mathrm{O} x$, il vient :

$$
\begin{gathered}
\iint_{(2)}\left(\frac{p}{\rho g}+\frac{u^{2}}{g}\right) d \sigma-\iint_{(1)}\left(\frac{p}{\rho g}+\frac{u^{2}}{g}\right) d \sigma \\
+\frac{1}{g} u(y, x, t) \overline{\mathrm{V}} \cdot \bar{n} d s \\
+\iiint_{(\delta)} \frac{1}{g} \frac{\partial u}{\partial t} d \tau=\left[i \eta-\frac{\tau_{0}}{\rho g}\right] d x
\end{gathered}
$$

L'écoulement étant plan, le fluide est supposé limité par deux plans verticaux parallèles à $O x$ et distants de l'unité de longueur. $\tau_{0}$ est la force de frottement sur le radier par unité de surface.

p $u(y, x, t) \bar{V} . \bar{n} d s$ représente le débit de quantité de mouvement à travers l'élément $1 \times d s$ de la surface libre compris entre les sections (1) et (2). Nous avons vu que :

$\bar{V} \cdot \bar{n} d s=\left[w(y, x, t)-u(y, x, t) \frac{\partial y}{\partial x}\right] d x=\frac{\partial y}{\partial t} d x$

Ce débit de quantité de mouvement a donc pour valeur :

$$
u(y, x, t) \frac{\partial !}{\partial t} d x
$$

l’intégrale triple :

$$
\iiint_{(\delta)} \frac{1}{g} \frac{\partial u}{\partial t} d \tau
$$

est étendue au volume du fluide compris entre les sections (1) et (2) et les deux plans verticaux parallèles à $O x$ et distants de l'unité. (1) et (2) étant infiniment voisins et l'écoulement plan, cette intégrale se réduit à l'intégrale simple :

$$
\frac{d x}{g} \int_{0}^{y} \frac{\partial u}{\partial t} d z
$$

quant à l'intégrale double :

$$
\iint_{(1)}\left(\frac{p}{\rho g}+\frac{u^{2}}{g}\right) d \sigma
$$

étendue à l'aire de la section (1), elle est donnée par l'intégrale simple :

$$
\int_{0}^{y}\left(\frac{p}{\rho g}+\frac{u^{2}}{g}\right) d z
$$

Nous poserons, comme dans l'étude des mouvements permanents :

$$
\mathrm{M}(x, t)=\int_{0}^{g}\left(\frac{p}{\rho g}+\frac{n l^{2}}{g}\right) d z
$$

Dans ces conditions, le théorème des quantités de mouvement en projection sur $\mathrm{O} x$ s'écrit :

$\frac{\partial \mathrm{M}}{\partial x}+\frac{1}{g} u(y, x, t) \frac{\partial y}{\partial t}+\frac{1}{g} \int_{0}^{y} \frac{\partial u}{\partial t} d z=i y-\frac{\tau_{0}}{\rho g}$

en se reportant à la formule de définition du débit par unité de longueur :

$$
q=\int_{0}^{y} u d z
$$

on voit immédiatement que la formule de dérivation sous le signe somme donne:

$$
\frac{\partial q}{\partial t}=\int_{0}^{\nu} \frac{\partial u}{\partial t} d z+u(y, x, t) \frac{\partial y}{\partial t}
$$

et l'équation précédente se réduit à :

$$
\frac{\partial \mathrm{M}}{\partial x}+\frac{1}{g} \frac{\partial q}{\partial t}=i y-\frac{\tau_{0}}{p g}
$$

Pour le calcul de l'intégrale :

$$
\int_{0}^{\prime \prime}\left(\frac{p}{\rho g}+\frac{u^{2}}{g}\right) d z
$$

nous admettrons que la répartition des pressions dans la section normale (1) d'abscisse $x$ est la même que si le fluide était parfait; elle est donc donnée par l'équation:

$$
\frac{1}{\rho g}-\frac{\partial p}{\partial z}=-1-\frac{u}{g} \frac{\partial w}{\partial x}-\frac{w}{g} \frac{\partial w}{\partial z}--\frac{1}{g} \frac{\partial w}{\partial t}
$$

Quant à la répartition des vitesses normales $u$ dans la section, nous la supposerons également donnée par la relation :

$$
\frac{u(z, x, t)}{\mathrm{U}(y, t)}=\mathrm{F}^{\prime}\left(\frac{z}{y}\right)
$$

où $U$ désigne la vitesse moyenne dans la section et $F(\alpha)$ une fonction dont la dérivée caractérise la courbe de répartition des vitesses en coordonnées sans dimensions. Ceci revient à admettre que cette courbe est indépendante de la section envisagée.

$F(x)$ ne peut d'ailleurs être choisie arbitrairement. En effet :

$$
q=\mathrm{U} y=\int_{0}^{y} u d z=\mathrm{U} \int_{0}^{y} \mathrm{~F}^{\prime}\left(\frac{z}{y}\right) d z
$$

ou en posant :

$$
\frac{z}{y}=\% \quad 1=\int_{0}^{1} \mathrm{~F}^{\prime}(\alpha) d \alpha
$$

ou en prenant pour $F(y)$ la fonction primitive de $F^{\prime}(\alpha)$ qui s'annule pour : $\alpha=0 \quad F(1)=1$.

Nous avons done $F(0)=0 \quad F(1)=1$. 
Dans ces conditions, la composante verticale de la vitesse se calcule à partir de l'équation de continuité :

$$
\frac{\partial u}{\partial x}+\frac{\partial w}{\partial z}=0
$$

en faisant intervenir la fonction de courant $\downarrow(z, x, t)$ qui satisfait aux deux relations :

$$
u=\frac{\partial \psi}{\partial z} \quad w=-\frac{\partial \psi}{\partial x}
$$

on a done :

$$
\frac{\partial \psi}{\partial z}=u=\mathrm{UF}^{\prime}\left(\frac{z}{y}\right)=\frac{q(x, t)}{y} \mathrm{~F}^{\prime}\left(\frac{z}{y}\right)
$$

ou en intégrant :

$$
\psi=q \mathrm{~F}\left(\frac{z}{y}\right)+f(x, t)
$$

la fonction arbitraire $f(x, t)$ étant déterminéc par les conditions aux limites. On en déduit :

$$
\begin{aligned}
w=-\frac{\partial \psi}{\partial x}=\frac{q}{y^{2,}} z \frac{\partial y}{\partial x} & \cdot \mathrm{F}^{\prime}\left(\frac{z}{y}\right) \\
& -\frac{\partial q}{\partial x} \mathrm{~F}\left(\frac{z}{y}\right)+f_{m}^{\prime}(x, t)
\end{aligned}
$$

Il reste à satisfaire atux conditions, aux limites.

Au fond $z=0$, on doit avoir : $w=0$;

En tenant comple de la condition $\mathbf{F}(0)=0$, il vient :

$$
f_{x}^{\prime}(x, t)=0
$$

par suite :

$$
w=\frac{q z}{y^{2}} \frac{\partial y}{\partial x} \mathrm{~F}^{\prime}\left(\frac{z}{y}\right)-\frac{\partial q}{\partial x} \mathrm{~F}\left(\frac{z}{y}\right)
$$

qui peut également s'écrire :

$$
w(z, x, t)=u(z, x, t) \frac{\partial y}{\partial x} \times \frac{z}{y}-\frac{\partial q}{\partial x} \mathrm{~F}\left(\frac{z}{y}\right)
$$

Sur la surface libre $z=y, w$ doit également satisfaire à la relation $(20)$ :

$$
w(y, x, t)=u(y, x, t) \frac{\partial y}{\partial x}+\frac{\partial y}{\partial t}
$$

cette condition, compte tenu de $F(1)=1$, conduit précisément à l'équation (21) :

$$
\frac{\partial y}{\partial t}+\frac{\partial q}{\partial x}=0
$$

qui apparaît ainsi comme une condition aux limites de l'équation de continuité.
La répartition des vitesses dans la section d'abscisse $x$ est ainsi donnée par les relations :

$$
\begin{gathered}
u=\frac{q}{y} \mathbf{F}^{\prime}\left(\frac{z}{y}\right) \\
w=q \frac{z}{y^{2}} \frac{\partial y}{\partial x} \mathbf{F}^{\prime}\left(\frac{z}{y}\right)-\frac{\partial q}{\partial x} \mathbf{F}\left(\frac{z}{y}\right)
\end{gathered}
$$

La répartition des pressions dans la section s'obtient en reportant les valeurs de $u$ et $w$ dans l'équation :

$$
\frac{1}{\rho g} \frac{\partial p}{\partial z}=-1-\frac{u}{g} \frac{\partial w}{\partial x}-\frac{w}{g} \frac{\partial w}{\partial z}-\frac{1}{g} \frac{\partial w}{\partial t}
$$

L'intégration, compie tenu de la condition à la surface libre $p=0$ pour $z=y$, nous donnera la valeur de la pression en tout point de la section.

Le calcul se poursuit de la mème facon que pour les écoulements permanents et donne en définitive la valeur de la quantité.

$$
\mathrm{M}(x)=\int_{0}^{1 /}\left(\frac{p}{\rho g}+\frac{u^{2}}{g}\right) d z
$$

en fonction de $q$, de $y$ et de leurs dérivées

Nous nous placerons désormais dans l'hypothese d'une répartition uniforme des vitesses dans la section. Cette hypothèse est valable pour l'étude de la houle et des intumescences de longueur variable dans un canal de faible profondeur, phénomènes que nous nous proposons d'étudier; elle ne s'appliquerait pas par contre à l'étude de la houle en eau profonde, mais on pourrait dans ce cas admettre une répartition des ritesses plus conforme à la réalité.

Cela revient à poser :

$$
F^{\prime}(\alpha)=1 \quad F(\alpha)=\alpha
$$

Les calculs se simplifient considérablement en prenant pour fonctions inconnues, au lieu du débit $q(x, t)$, la composante horizontale de la vitesse $u(x, t)$ dans la section envisagée qui coïncide ici avec la vitesse moyenne $\mathrm{U}(x, t)$.

La composante verticale de la vitesse $w$ a alors pour valeur :

$$
w=q \frac{z}{y^{2}} \frac{\partial y}{\partial x}-\frac{z}{y} \frac{\partial q}{\partial x}
$$

ou, en tenant compte de la relation $q=u y$ :

$$
w=-z \frac{\partial u}{\partial x}
$$

en portant dans la relation :

$$
\frac{1}{i g} \frac{\partial p}{\partial z}=-1-\frac{u}{g} \frac{\partial w}{\partial x}-\frac{w}{g} \frac{\partial w}{\partial z}-\frac{\partial w}{\partial t}
$$


on obtient :

$\frac{1}{* g} \frac{\partial p}{\partial z}=-1-z\left[\left(\frac{\partial u}{\partial x}\right)^{2}-u \frac{\partial^{2} u}{\partial x^{\prime \prime}}-\frac{\partial^{2} u}{\partial x \partial t}\right]$

L'intégration, compte tenu de la condition à la surface $p=0$ pour $z=: y$, conduit à l'expression :

$\frac{l}{\rho g}=--z+y+\frac{y^{2}-z^{2}}{2 g}\left[\left(\frac{\partial u}{\partial x}\right)^{2}-u \frac{\partial^{2} u}{\partial x^{\prime 2}}-\frac{\partial^{2} u}{\partial x \partial t}\right]$

Le calcul de l'intégrale :

$$
\int_{0}^{y}\left(\frac{p}{g}+\frac{u^{2}}{g}\right) d z
$$

s'effectue alors immédiatement; on obtient :

$$
\begin{aligned}
M(x)=\frac{y^{2}}{2}-\frac{u^{2} y}{g} & \\
& +\frac{y^{:}}{3 g}\left[\left(\frac{\partial u}{\partial x}\right)^{2}-u \frac{\partial^{2} u}{\partial x^{2}}-\frac{\partial^{2} u}{\partial x \partial t}\right]
\end{aligned}
$$

Les équations aux dérivées parlielles de la ligne d'eau s'écrivent alors, compte tenu de la valeur de la force de frottement sur le radier :

$$
\begin{aligned}
& \mathbf{M}=\frac{y^{2}}{2}+\frac{u^{2} y}{g}+\frac{y^{3}}{3 g}\left|\left(\frac{\partial u}{\partial x}\right)^{2}-u \frac{\partial^{2} u}{\partial x^{\prime}}-\frac{\partial^{2} u}{\partial x \partial t}\right| \\
& \frac{\partial \mathrm{M}}{\partial x}+\frac{1}{g} \frac{\partial}{\partial t}(u y)=i y \frac{u^{2}}{c^{2}(y)} \\
& \frac{\partial y}{\partial t}+\frac{\partial(u y)}{\partial x}=0
\end{aligned}
$$

Les équations (22) constituent une généralisation des équations de Barré-DE-SAINT-VENANT, lorsqu'on tient compte de l'inclinaison et de la courbure des filets liquides.

Nous en ferons une application à l'étude des intumescences qui se propagent le long du canal avec une célérité constante $V$.

\section{2. - Equations différentielles des intumescences à célérité constante.}

Le temps $t$ et l'abscisse $x$ ne figurant pas explicitement dans les équations (22), ces dernières admettent des solutions où l'une quelconque des quantités $y, u, M$ est fonction de la seule variable $x-V t$, où $Y$ est une constante donnée qui caractérise la vitesse de propagation.

Ces solutions :

$y=y(x-\mathrm{V} t) \quad u=u(x-\mathrm{V} t) \quad \mathrm{M}=\mathrm{M}(x-\mathrm{V} t)$

sont les intégrales d'un système différentiel qui se déduit immédiatement des équations (22).

On a, en effet :

$$
\frac{\partial y}{\partial t}=-\mathrm{V} \frac{\partial y}{\partial x}
$$

La troisième équation (22) s'écrit alors :

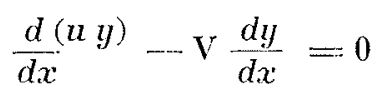

ou, en intégrant :

$$
u=\mathrm{V}+\frac{q}{y}
$$

$q$ étant une constante arbitraire dont la signification mécanique est évidente. $q$ est le débit du fluide par mètre linéaire à travers une sec- tion se déplaçant dans le canal avec une vitesse constante égale à la célérité de propagation $\mathrm{V}$.

En reportant la valeur de $u$ tirée de (23) dans la première équation (22), on obtient:

$$
\mathrm{M}=\frac{y^{2}}{2}+\frac{u^{2} y}{g}+\frac{q^{2}}{3 g}\left[\frac{d^{2} y}{d x^{2}}-\frac{1}{\eta}\left(\frac{d y}{d x}\right)^{2}\right]
$$

La deuxième équation s'écrit, par ailleurs, en tenant compte de la relation :

$$
\begin{gathered}
\frac{\partial}{\partial t}(u y)=-\mathrm{V} \frac{d}{d x}(u y)=-\mathrm{V}^{2} \frac{d y}{d x} \\
\frac{d}{d x}\left[\mathrm{M}-\frac{V^{2} y}{g}\right]=i y-\frac{u^{2}}{c^{2}(y)}
\end{gathered}
$$

La comparaison avec (24), écrite sous la forme:

$$
\begin{aligned}
\mathrm{M}-\frac{\mathrm{V} 2 y}{g}-2 q \mathrm{~V} & =\frac{y^{2}}{2}+\frac{q^{2}}{g !} \\
& +\frac{q^{2}}{3 g}\left[\frac{d^{2} y}{d x^{2}}-\frac{1}{y}\left(\frac{d y}{d x}\right)^{2}\right]
\end{aligned}
$$

nous conduit à faire intervenir la nouvelle fonction inconnue :

$$
\mathrm{F}=\mathrm{M}-\frac{\mathrm{V}^{2} y}{g}-2 q \mathrm{~V}
$$


et à mettre les équations (24) et (25) sous la forme :

$\left\{\begin{array}{l}\mathrm{F}=\frac{y^{2}}{2}+\frac{q^{2}}{g y}+\frac{q^{2}}{3 g}\left|\frac{d^{2} y}{d x^{2}}-\frac{1}{y}\left(\frac{d y}{d x}\right)^{2}\right|_{(26} \\ \frac{d \mathrm{~F}}{d x}=i y-\frac{1}{c^{2}(y)}\left[\mathrm{V}+\frac{q^{2}}{y}\right]^{2}\end{array}\right.$

dont l'analogie avec les équations du régime permanent est évidente.

Rrmarque. -- Cette analogie ne se poursuivrait pas dans l'hypothèse d'une répartition non uniforme de la vitesse dans une section :

L'étude des intumescences se progageant avec une vitesse constante le long d'un canal est dono absolument analogue à celle des lignes d'eau en régime permanent et elle justifie le fait de considérer comme des «ondes stationnaires " les ondulations de surface et le ressaut en régime permanent.

Nous donnerons une première application des équations (26) à la détermination des ondes de translation en deuxième approximation.

\section{3. - Théorie de la houle en eau courante en deuxième approximation.}

Considérons un canal rectangulaire de grande largeur de pente $i$, dans lequel est réalisé un écoulement permanent uniforme de vitesse $u_{0}$.

Nous désignerons par $h$ la hauteur d'eau dans le canal liée à $u_{0}$ et $i$ par la relation du régime normal :

$$
i h-\frac{u_{0}^{2}}{c^{2}(h)}=0
$$

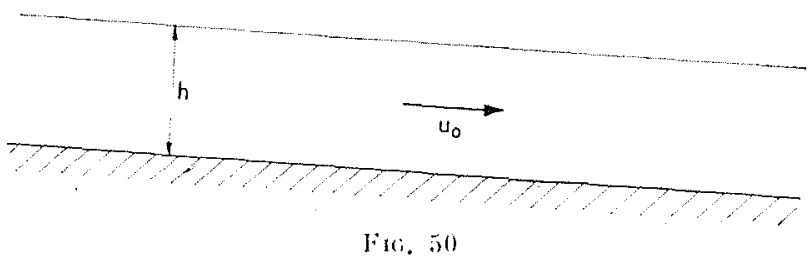

Nous supposerons la vitesse $u_{0}$ faible, de telle sorte que le régime soit fluvial dans le canal. Dans les phénomènes ondulatoires dont il est question ici, l'influence du frottement est négligeable de telle sorte que, dans une section ou la hauteur d'eau est $y$ et la vitesse $u$, la quantité $i y-\left(u^{2} / c^{2} y\right)$ est peu différente de 0 .

l)ans ces conditions, les ondes de translation sont définies par le sýstème différentiel :

$$
\left\{\begin{array}{l}
\mathrm{F}=\frac{y^{2}}{2}+\frac{q^{2}}{g y}+\frac{q^{2}}{3 g}\left[\frac{d^{2} y}{d x^{2}}-\frac{1}{y}\left(\frac{d y}{d x}\right)^{2}\right] \\
\frac{d \mathrm{~F}}{d x}=00
\end{array}\right.
$$

système qui a déjà été intégré dans l'étude du régime permanent. On est conduit à une équation différentielle du premier ordre qui définit, à un instant donné, le profil des ondes de translation :

$$
\frac{q^{2}}{6 g}\left(\frac{d y}{d x}\right)^{2}=\mathrm{C}_{1} y^{2}-\mathrm{F} y-\frac{y^{3}}{2}+\frac{q^{2}}{2 g}
$$

$\mathrm{C}_{1}, \mathrm{~F}$ et $q$ étant trois constantes.
Nous mettrons cette équation sous la forme :

$$
\frac{q^{2}}{3 g}\left(\frac{d y}{d x}\right)^{2}==\left(y-y_{0}\right)\left(y_{1}-y\right)\left(y-\frac{q^{2}}{g y_{1} y_{0}}\right)
$$

qui fait intervenir les ordonnées $y_{0}$ et $y_{1}$ des sommets de la ligne d'eau.

$V$ désignant la vitesse de propagation de la houle par rapport à des axes fixes, considérons une section ( $\supseteq$ ) se déplaçant dans le fluide avec la vitesse $V$, et soient respectivement y la hauteur d'eau et $"$ la composante horizontale de la vitesse absolue du fluide dans la section ( 2 ). Le débit par mètre linéaire à travers ( 2 ), c'est-à-dire la quantité d'eau qui traverse cette section par

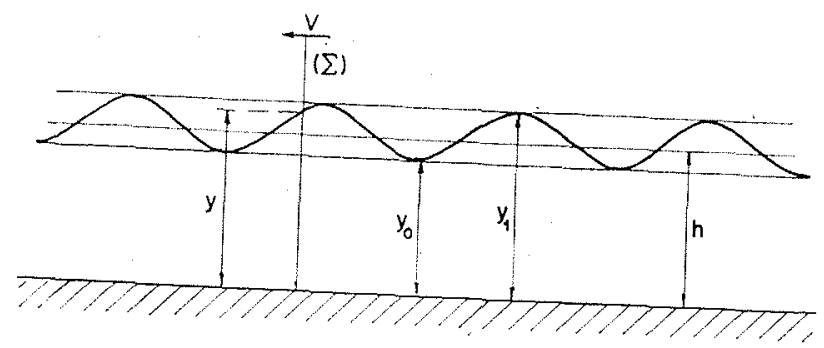

FIG, 51

unité de temps, est relié à $u, y, V$ par la relation (23):

$$
u=v+\frac{q}{y}
$$

en particulier pour : $y=h \quad u=u_{0}$ :

$$
u_{0}=\mathrm{V}+\frac{q}{h}
$$

nous sommes donc conduit à poser $q=-\mathrm{V}_{r} h$ :

$$
\mathrm{V}=u_{0}+\mathrm{V}_{v}
$$




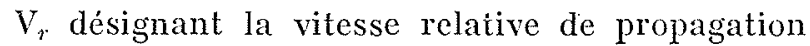
de la houle par rapport au courant de vitesse $u_{0}$.

L'équation (27) s'écrit dans ces conditions :

$$
\frac{V_{r}^{2} h^{2}}{3 g}\left(\frac{d !}{d x}\right)^{2}=\left(y-y_{0}\right)\left(y_{1}-y\right)\left(y-\frac{V_{r}^{2} h^{2}}{g y_{1} y_{0}}\right)
$$

et la demi-longueur d'onde de la houle est donnée par l'intégrale définis:

$$
\mathrm{L}=\frac{V_{r} h}{\sqrt{3 g}} \int_{\frac{d y_{0}}{\sqrt{1 / 2}}}^{\frac{d y}{\left(y-y_{0}\right)\left(y_{1}-y\right)\left(y-\frac{V_{r}^{2} h^{2}}{g y_{1} y_{0}}\right)}}
$$

qui, ainsi que nous l'avons vu dans l'étude des ondes stationnaires, se transforme à l'aide du changement de variable :

$$
y=\frac{y_{1}+y_{0}}{2}+\frac{y_{1}-y_{0}}{2} u
$$

en une intégrale elliptique d'un type classique:

$$
L=\frac{V_{r} h}{\sqrt{3 g\left(\frac{y_{1}+y_{0}}{2}-\frac{V_{r}^{2} h^{2}}{g y_{1} y_{0}}\right)}} \int_{-1}^{+1} \frac{d u}{\sqrt{\left(1-u^{2}\right)(1+\overline{\mathrm{K} u})}}
$$

avec :

$$
\mathrm{K}=\frac{y_{1}-\frac{y_{0}}{2 V_{r}^{2} h^{2}}}{g y_{1} y_{0}}
$$

Les paramètres caractéristiques de la houle, $\mathrm{L}, \mathrm{V}_{\mathrm{r}}, h, y_{1}, y_{0}$ ne sont d'ailleurs pas liés par la seule relation $(2 \delta)$.

Le phénomène de translation de la surface libre ne correspond pas en effet à un déplacement en masse du fluide, ce déplacement provenant uniquement du courant de vitesse $u_{0}$. Nous obtiendrons donc une nouvelle relation en écrivant que la quantité d'eau qui traverse, pendant une période $T$, une section fixe déterminée du fluide, est la même que si la houle n'existait pas, ce qui conduit à écrire :

or :

$$
\int_{t}^{t+\mathrm{T}} u y d t=u_{0} h \mathrm{~T}
$$

$$
u y=\mathrm{V} y+q=\mathrm{V}(y-h)+u_{0} h
$$

on en déduit :

$$
\int_{t}^{t+\mathrm{T}}(y-h) d t=0
$$

Dans la section d'abscisse $x_{0}$, $y$ étant une fonc- tion de $x_{0}-V t$, un changement de variable évident conduit à la relation :

$$
\int_{x_{0}}^{x_{0}+2 \mathrm{~L}}(y-h) d x=0
$$

qui exprime que $h$ est l'ordonnée moyenne de la ligne d'eau.

De la symétrie de la ligne d'eau on déduit la relation équivalente :

$$
\int_{x_{0}}^{x_{0}+\mathrm{L}} y d x=h \mathrm{~L}
$$

$x_{0}$ étant l'abscisse d'un sommet de la surface libre.

Or, dans l'intervalle $x_{0}, x_{0}+\mathrm{L}, x$ est une fonction uniforme de $y$, définie par l'équation à va-

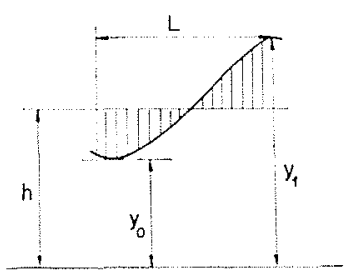

FIG. 52

riable séparée $(d y / d x)=\wp(y)$ déduite de $(27)$ en choisissant la détermination convenable.

On peut donc prendre y comme variable d'intégration et écrire :

$$
h=\frac{\int_{y_{0}}^{y_{1}} \frac{y d y}{\varphi(y)}}{\int_{y_{0}}^{y_{1}} \frac{d y}{\varphi(y)}}
$$

avec :

$$
(y)=\frac{\sqrt{3 g}}{V_{r} h} \sqrt{\left(y-y_{0}\right)\left(y_{1}-y\right)\left(y-\frac{V_{r}^{2} h^{2}}{g y_{1} y_{0}}\right)}
$$

Il est intéressant d'utiliser le changement de variable :

$$
y=\frac{y_{1}+y_{0}}{2}+\frac{y_{1}-y_{0}}{2} u
$$

qui conduit à une relation entre les deux quantités sans dimensions :

$$
\begin{aligned}
\mathrm{K} & =\frac{y_{1}-y_{0}}{y_{1}+y_{0}-\frac{2 \mathrm{~V}_{r}{ }^{2} h^{2}}{g y_{1} y_{0}}} \\
\lambda & =\frac{y_{1}+y_{0}-2 h}{y_{1}-y_{0}}
\end{aligned}
$$


on obtient, tous calculs faits :

$$
\lambda=-\frac{\int_{-1}^{+1} \frac{u d u}{\sqrt{\left(1-u^{2}\right)(1+K u)}}}{\int_{-1}^{+1} \frac{d u}{\sqrt{\left(1-u^{2}\right)(1+K u)}}}=\psi(K)
$$

Le paramètre $\mathrm{K}$ est évidemment positif puisque nous avons supposé $y_{1}>y_{0}$ et que $V_{r}$ doit salisfaire à la relation évidente d'après (28):

$$
y_{1}+y_{0}-\frac{2 V_{r^{2}} h^{2}}{g y_{1} y_{0}}>0
$$

les intégrales définies, qui figurent dans (29), n'ayant un sens que pour $-1<\mathrm{K}<1$, le paramètre $K$ est nécessairement compris entre 0 et 1 .

La fonction $\lambda=\psi(K)$ est donc définie et continue dans l'intervalle 0 et 1 , la borne 1 étant exclue. Pour $\mathrm{K}=1$, les intégrales figurant dans

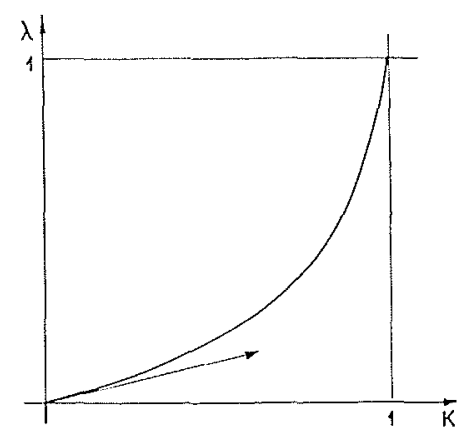

Fic. 53

(29) n'ont en effet pas de sens. Cependant elles tendent vers l'infini lorsque $K$ tend vers 1 par valeurs inférieures et il est facile de montrer que $\lambda$ tend alors vers 1 .

L'équation (29) peut s'écrire en effet :

$$
\lambda=1 \frac{\int_{-1}^{+1} \frac{(1+u) d u}{\sqrt{\left(1-u^{2}\right)(1+\mathrm{K} u)}}}{\int_{-1}^{+1} \frac{d u}{\sqrt{\left(1-u^{2}\right)(1+\mathrm{K} u)}}}=1-\frac{f(\mathbf{K})}{g(\mathrm{~K})}
$$

et on voit aussitôt que lorsque $K$ tend vers 1 par valeurs inférieures, $f(\mathrm{~K})$ tend vers $\pi, g(\mathrm{~K})$ tend vers $+\infty, \lambda$ a donc pour limite 1 .

$\lambda$ est d'ailleurs défini dans l'intervalle $-1+1$ par le développement uniformément convergent :

$$
\lambda=\frac{K}{4} \quad+\ldots
$$

la courbe représentative de la fonction $\lambda=\Psi(\mathrm{K})$ est tracée sur la figure 53.

A une valeur de $\lambda$, comprise entre 0 et 1 , correspond done une valeur de $K$ unique, égale- ment comprise entre 0 et 1 et, par suite, deux valeurs de la vitesse relative de propagation $\mathrm{V}$, égales et de signe contraire. La longueur d'onde de la houle correspondanie $2 \mathrm{~L}$ est alor's donnée par la relation (28).

Dans un canal rectangulaire de grande largeur où est réalisé un régime permanent uniforme de vitesse $u_{0}$ correspondant à une hauteur d'eau $h$, la vitesse relative $V_{r}$ de propagation et la longueur d'onde d'une houle, caractérisée par les ordonnées $y_{0}$ et $y_{1}$ de ses sommets, sont donc parfaitement définies par les équations (28) et $(29)$.

II existe deux valeurs égales et de signe contraire de la vitesse relative de propagation $V_{\text {, }}$, correspondant respectivement à une houle monlante et à une houle descendante.

$V_{r}$ est indépendante de la vitesse $u_{0}$ du régime permanent et uniforme auquel la houle est superposée, La vitesse absolue de propagation s'obtient par la règle de composition des vitesses, d'après la relation précédemment démontrée:

$$
\mathrm{V}=u_{0}+\mathrm{V}_{r}
$$

Avant d'examiner la forme de la houle, dans le cas général, nous étudierons deux cas particuliers importants correspondant aux valeurs extrêmes du paramètre $\lambda: 1$ et 0 .

Cas $\lambda=1$. ONde solitarre.

Dans lhypothèse où $\lambda=1$

$$
\frac{y_{1}+y_{0}-2 h}{y_{1}-y_{0}}=1,
$$

on en déduit $y_{0}=h$. L'onde est située tout entière au-dessus de la surface libre, sa vitesse de propagation se déduit de :

$$
\mathrm{K}=\frac{y_{1}-y_{0}}{y_{1}+y_{0}-\frac{2 V_{1}{ }^{2} h^{2}}{g y_{1} y_{0}}}=1
$$

dans laquelle on fait $h=y_{0}$.

On obtient: $\mathrm{V}_{r^{2}}{ }^{2}=g y_{1} \quad \mathrm{~V}_{r}= \pm \sqrt{g y_{1}}$

La longueur d'onde $L$ est infinie avec l'intégrale :

$$
\int_{-1}^{+1} \frac{d u}{\sqrt{\left(1-u^{2}\right)(1+K u)}} \text { pour } K=1
$$

Le raccordement avec le niveau primitif ( $\left.y=y_{0}=h\right)$ n'est done réalisé théoriquement qu'à l'infini amont et aval. L'équation de l'onde montre qu'en fait le raccordement est pratique- 


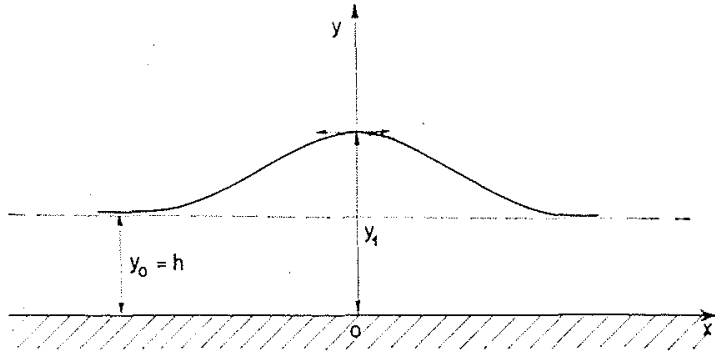

FIG. 54

ment réalisé à une distance faible du sommet de l'onde.

L'équation différentielle (27) qui définit la ligne d'eat s'écrit en effet, lorsqu'on y fait $V_{r}=g y_{1}, \quad h=y_{0}$

$$
\frac{\left.y_{1} y_{0}\right)^{\prime \prime}}{3}\left(\frac{d y}{d x}\right)^{3}=\left(y-y_{0}\right)=\left(y_{1} \cdots-y\right)
$$

Cette équation a déjà élé infégrée au cours de l'étude des mouvements permanents. Toutes les courbes intégrales se déduisent de l'une d'entre elles par une translation parallèle à $\mathrm{O} x$.

L'équation de cette courbe par rapport aux axes $O x$, et $O y$ roy passant par le sommet $y=y_{1}$ (fig. 54) s'écrit :

$$
y=y_{1}+4\left(y_{1}-y_{0}\right) \frac{e^{\left(x+1 / y_{n}\right.}}{\left(1+e^{a \cdots / y_{1}}\right)^{2}}
$$

a étant un coefficient sans dimension qui a pour valeur :

$$
\alpha=\sqrt{3\left(1-\frac{y_{11}}{y_{1}}\right)}
$$

On retrouve ainsi les résultats obtenus par Borssinese dans ses études sur l'onde solitaire. Ce dernier adoptait les notations suivantes :

$$
y_{0}=\mathrm{H}, \quad y-y_{0}=h, \quad y_{1}-y_{0}=h_{1}
$$

et obtenait pour équation de l'onde solitaire :

$$
\frac{\mathrm{H}^{3}}{3}\left(\frac{d h}{d x}\right)^{2}=h^{2}\left(h_{1}-h\right)
$$

Avec les mèmes notations, notre équation s'écrit :

$$
\frac{\mathrm{H}^{2}\left(\mathrm{H}+h_{1}\right)}{3}\left(\frac{d h}{d x}\right)^{2}=h^{2}\left(h_{1}-h\right)
$$

qui coüncide bien avec l'équation précédente lorsque $h_{1}$ est voisin de 0 , c'est-à-dire pour une onde de faible amplitude. Boussıneso se plaçait précisément dans cette hypothèse. BAzIN, dans ses études expérimentales, ayant vérifié que l'onde solitaire conservait sa forme caractéristique, sans déferlement, tant que $y_{1}$ est inférieur à $2 h$, on voit que nos équations représentent bien le phénomène alors même quion s’éloigne des hypothèses de calcul admises par Boussineso.

Celui-ci supposait également que la vitesse de l'eau dans le canal ctait nulle avant le passage de l'onde. Dans le cas d'une houle superposée à un courant de vitesse $u_{0}$, la règle de composition des vitesses émises par lui se déduisait des équations de BARRÉ-DE-SAINT-VENANT, dans lesquelles la courbure de l'onde est négligée. Nos équations montrent que cette regle reste valable en deuxième approximation, à condition que la répartition des vitesses dans me section soit sensiblement uniforme. Cetle hypothese ne correspond pas à la réalité dans le cas où l'amplitude de l'onde est trop importante.

Les relations (28) el (29) nous permettent égaIement de faire une autre remarque sur les développements classiques concernant les ondes de translation en deuxième approximation. On peut se demander en effet pour quelle raison ces développements, qui posent, dans sa généralité, te probleme de la recherche des ondes de translation de faible amplitude à célérité constante, conduisent en fait uniquement à l'onde solitaire. Cette raison semble résider dans l'hypothèse, faite par Botssineso, que pour une onde de faible amplitude la vitesse de propagation est donnée par les lois de premiere approximation, hy pothese qui est valable miquement dans le eas de l'onde solitaire.

Lóquation (29) $\lambda=\psi(K)$ montre en efrel que, dans un canal ou est réalisé un régime permament uniforme de vitesse $u_{0}$ correspondant à une hauteur d'eau $h$, il pcut exister une infinité do houles d'amplitude donnée $y_{1}-y_{0}=h_{1}$, chacune de celles-ci ayant une célérité relative $V$, et une longueur d'onde $2 \mathrm{~L}$ différente. L'onde solitaire est précisément celle d'entre elles dont la vitesse de propagation:

$$
V_{r}= \pm \sqrt{g y_{1}}= \pm \sqrt{g\left(h+h_{1}\right)}
$$

est maximum. Elle correspond à $: \mathrm{K}=1 \quad \lambda=1$.

Envisageons maintenant le cas où le paramètre $\lambda$ a une valeur voisine de 0 . Il en est alor's de mème d'après (29) pour le paramètre:

$$
\mathbf{K}=\frac{y_{1}-y_{0}}{y_{1}+y_{0}-\frac{2 \mathrm{~V}_{r}{ }^{2} h^{2}}{g y_{1} y_{0}}}
$$

ce qui nécessite une onde de faible amplitude $y_{1}-y_{0}$. (Cette condition n'est d'ailleurs pas suffisante puisque, dans le cas de l'onde solitaire :

$$
y_{1}+y_{0}-\frac{2 V_{r}^{2} h^{2}}{g y_{1} y_{0}}
$$

est équivalent à $y_{1}-y_{11}$ ) 
Le paramètre :

$$
\lambda=\frac{y_{1}+y_{1}-2 h}{y_{1} \cdots y_{0}}
$$

étant voisin de 0 et $y_{1}-y_{0}$ petit, cela signifie que si l'on considère $y_{1}-y_{0}$ comme un infiniment petit, $y_{1}+y_{0}-2 h$ doit ètre un infiniment petit du second ordre par rapport à $y_{1}-y_{0}$.

On a alors affaire à une houle sensiblement sinusoïdale, dont la vitesse de propagation est indépendante de l'amplitude; K étant petit, on peut, en effet, dans l'équation (28), remplacer l'intégrale:

$$
\int_{-1}^{+1} \frac{d u}{\sqrt{\left(1-u^{2}\right)(1+K u)}}
$$

par le premier terme de son développement en fonction de $K$ et poser :

$$
y_{1}+y_{0}=2 h \quad y_{1} y_{0}=h h^{2}
$$

Dans ces conditions, (28) s'écrit:

$$
\mathrm{L}=\frac{\pi \mathrm{V}_{r} h}{\sqrt{3 g h-3 \mathrm{~V}_{r^{2}}}}
$$

c'est-it-dire:

$$
\mathrm{V}, 2=\frac{g h}{1+\frac{\pi^{2}}{3} \frac{h^{2}}{\mathrm{~L}^{2}}}
$$

oll :

$$
\mathrm{V}_{r}=\sqrt{g h\left(1-\frac{\pi^{2}}{3} \frac{h^{2}}{\mathrm{I}^{2}}+\cdots\right)}
$$

qui, pour $h / \mathrm{L}$ petit, constitue une approximation au troisieme ordre près de la formule classique donnant la célérité de la houle en profondeur finie':

$V=\sqrt{g \frac{\mathrm{L}}{\pi}}$ th $\frac{\pi h}{\mathrm{~L}}=\sqrt{g h\left(1-\frac{\pi^{2}}{3} \frac{h^{2}}{\mathrm{~L}^{2}}+\cdots\right)}$

Cetle formule s'obtient à partir des équations générales de l'hydrodynamique en linéarisant les conditions aux limites, en négligeant les earrés et les produits des vitesses et dans l'hypothèse d'une très faible amplitude. Cette houle trochoïdale, dont la vitesse de propagation est indépendante de l'amplitude, constitue une solution du problème, mais ce n'est pas la seule. Il en existe une infinité d'autres, parmi lesquelles l'onde solitaire dont la vitesse de propagation :

$$
V_{r}=\sqrt{g y_{1}}=\sqrt{g\left(h+h_{3}\right)}
$$

n'est pas indépendante de l'amplitude.

En conclusion, les équations (28) el (29) permettent de déterminer la célérité et la longueur d'onde d'une houle d'amplitude non négligeable, superposée à un courant de vitesse $u_{0}$, lorsque la répartition des vitesses dans une section est sensiblement uniforme.

Elles ne s'appliquent pas à la houle en profondeur infinie, ni aux ondulations donl la hauldur est trop grande.

\section{4. - Intumescences de grande longueur où l'influence du frottement n'est pas négligeable.}

L'étude des intumescences se propageant dans un canal rectangulaire de grande largeur avec une célérité constante $V$, se ramène à l'integration du système différentiel (26):

$$
\begin{gathered}
\mathrm{F}=\frac{y^{2}}{2}+\frac{q^{2}}{g y}+\frac{q^{2}}{3 g}\left[\frac{d^{2} y}{d x^{2}}-\frac{1}{y}\left(\frac{d y}{d x}\right)^{2}\right] \\
\frac{d \mathrm{~F}}{d x}=i y-\frac{u^{2}}{c^{2}(y)} \\
u=\mathrm{V}+\frac{q}{y}
\end{gathered}
$$

qui se réduit au système (8) lorsqu'on y fait $\mathrm{V}=0$.

Comme dans l'étude des mouvements permanents nous remplacerons la fonction $F$ par une nouvelle fonction auxiliaire $\Phi$ liée à $\mathrm{F}$ par la relation :

$$
\frac{d \mathrm{~F}}{d x}=y \frac{d \Phi}{d x}
$$

On est ainsi conduit au système équivalent à (26):

$$
\left\{\begin{array}{l}
\Phi=y+\frac{q^{2}}{2 g y^{2}}+\frac{q^{2}}{3 g y}\left[\frac{d^{2} y}{d x^{2}}-\frac{1}{2 y}\left(\frac{d y}{d x}\right)^{2}\right] \\
\frac{d \Phi}{d x}=i-\frac{u^{2}}{y c^{2}(y)} \\
u=V+\frac{q}{y}
\end{array}\right.
$$

La forme des intumescences de grande longueur à célérité constante est donc identique à celle des lignes de remous en régime permanent. 
Considérons un canal rectangulaire de grande largeur de pente $i$, dans lequel est réalisé un écoulement permanent uniforme de vitesse $u_{0}$. Nous désignerons par $y_{0}$ la hauteur normale du régime uniforme reliée à $u_{0}$ et à $i$ par la relation :

$$
i-\frac{u_{0}^{2}}{c^{2}\left(y_{0}\right) y_{0}}=0
$$

Nous nous proposons de déterminer les solutions du système (30) yui se raceordent avec le régime uniforme précédemment défini, e'est-àdire qui admettent comme asymptote la droite $y=y_{0}$

l.e problème ainsi posé se ramène à la recherche des solutions de (30) asymptotiques à la solution particulière:

$$
\begin{aligned}
& y=y_{0} \\
& \Phi=\Phi_{0}=y_{0}+\frac{q^{2}}{2 g y_{0}^{2}}
\end{aligned}
$$

$q$ étant une constante relice à la vitesse absolue de propagation de l'intumescence par la relation :

$$
q=\left(u_{0}-V\right) y_{0}
$$

Il ne différe de celui résolu dans létude du mouvement permanent à propos des équations (9), que par la forme de la fonction $\downarrow(y)$ qui intervient dans les équations $\left(10_{a}\right)\left({ }^{*}\right)$.

La fonction $\downarrow(y)$ s'ecrit ici :

$\downarrow(y)=i-\frac{u^{2}}{c^{2}(y) !}=i-\frac{1}{y c^{2}(y)}\left(V+\frac{q}{y}\right)^{2}$

alors qu'on avait en régime permanent :

$$
\psi(y)=i-\frac{q^{2}}{c^{2}(y) y^{3}} \quad \text { en posant } q=\frac{\mathrm{Q}}{l}
$$

L'existence de solutions de (30) asymptotiques à $y=y_{0}$ est ainsi liée au signe des parties réel les des racines de l'équation caractéristique du système linéaire qui constitue les «équations aux variations $\gg$ de $(30)$ au voisinage de la solution connue : $y=y_{0}$.

Cette équation caractéristique $\left(13^{\prime}{ }_{n}\right)$ s'écrit $\left(^{\star}\right)$ :

$$
\frac{q^{2}}{3 g} m^{3}+\left(y_{0}-\frac{q^{2}}{g y_{0}^{2}}\right) m=y_{0} \bigcup^{\prime}\left(y_{0}\right)\left(13_{n}^{\prime}\right)
$$

dans le calcul de $\psi^{\prime}\left(y_{0}\right)$ nous négligerons la variation du coefficient de CHÉzY. En tenant compte de la relation $q=\left(u_{0}-V\right) y_{0}$, on trouve :

$$
\psi^{\prime}\left(y_{0}\right)=\frac{u_{0}}{y_{0}^{2} c^{2}\left(y_{0}\right)}\left(3 u_{0}-2 V\right)
$$

(*) Cf. la Hoaille Blanche, no 3, 1953, p. 380 $\psi^{\prime}\left(y_{0}\right)$ ne peut donc être négatif que pour des intumescences dont la célérité est supérieure à une

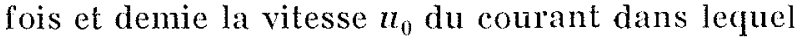
elles se propagent.

Nous nous bornerons à étudier ici les intumescences qui se déplacent vers l'amont $[\mathrm{V}<0]$ pour lesquelles $\psi^{\prime}\left(y_{0}\right)$ est obligatoirement positif quelle que soit leur célérité. live.

La constante $q=\left(\boldsymbol{u}_{0}-V\right) y_{0}$ est alors posi-

L'équation caractéristique $\left(13_{n}\right)$ admet alors, comme nous l'avons vu, une racine réelle positive $m_{1}$, deux racines réelles ou imaginaires $m$. et $m_{3}$ dont la partie réelle est négative.

Il existe donc une simple infinité de solutions dù système (30) asymptotiques à la solution par. ticulière $y=y_{0}, \Phi=\Phi_{0}$ lorsque $x$ augmente indéfiniment par valeurs négatives. Ces solutions se déduisent toutes de l'une d'entre elles par une translation parallèle à l'axe $O x$. Elles sont définies par les équations :

$$
y=y\left(x-\mathrm{C}_{1}\right) \quad \Phi=\Phi\left(x-\mathrm{C}_{1}\right)
$$

$y(\boldsymbol{u})$ et $\Phi(u)$ étant des fonctions parfaitement déterminées dont la forme dépend uniquement de la célérité $V$.

Quant au parametre de translation, il est nécessairement de la forme $C_{1}=x_{0}+V\left(t-l_{0}\right)$ puisque les équations (30) définissent les solutions de (22) qui sont fonctions de la senle variable indépendante $x-\mathrm{Vt}$.

Par un choix convenable de l'origine des abscisses et du temps, on peut donc écrire :

$$
y=y(x-V t) \quad \check{\Phi}=\Phi(x-V t)
$$

Dans le plan $O y$, ces solutions sont représentées par une courbe unique $\left(\mathrm{C}_{0}\right)$ définie paramétriquement par les équations précédentes. L'allure de cette courbe $\left(\mathrm{C}_{0}\right)$ a déjà été précisée dans l'étude du régime permanent. Elle passe par le point $\mathrm{P}$, intersection de la droite $y=y_{0}$ avec la courbe $(\mathbf{H})$ d'équation :

$$
\Phi=y+\frac{q^{2}}{2 g y^{2}}
$$

la pente de la tangente en $P$ à $\left(C_{0}\right)$ est :

$$
\left(\frac{d H}{d y}\right)_{y=y_{0}}=\frac{\psi^{\prime}\left(y_{0}\right)}{m_{1}}
$$

Les développements en série asymptotique de Poincaré et Liapounofr permettent également de développer $y(x-V t), \mathbb{\Phi}(x-\mathrm{V} t)$ au voisinage de $P$ en séries entières uniformément convergentes de la quantité $u=e^{m_{1} t r-v o}$. Nous n'in- 
sisterons pas sur ce calcul eflectué au chapitre III $\left(^{*}\right)$.

La courbe $\left(\mathrm{C}_{0}\right)$ est, nous l'avons vu, asymptote, lorsque $x$ augmente indéfiniment par valeur positive, à la courbe $(\mathrm{H})$ d'équation $\Phi=y+\left(q^{2} / 2 g y^{2}\right)$ et la ligne d'eau d'équation $y=y(x-y t)$ admet une asymptote horizontale.

r'aspect de l'intumeseence se déduit du trace de la courbe $\left(\mathrm{C}_{0}\right)$ et comme dans l'ćtude du régime permanent nous serons conduit à distinguer deux cas suivant que le point $P$ se trouve sur la branche fluviale ou torrentielle de $(\mathbf{H})$.

PRlEMLR CAS :

$$
y_{0}>y_{c}=\sqrt[3]{\frac{q^{2}}{g}}
$$

P sur la branche fluviale de $(\mathrm{H})$.

c'est-à-dire $q<y_{0} \vee \bar{g} y_{0}$, puisque $q>0$ ou, en tenant compte de la relation : $q=\left(u_{0}-V\right) y_{0}$ :

$$
\underline{V} \geq a_{0}-\sqrt{g y_{0}}
$$

Comme nous etudions ici les intumescences qui remontent le courant $[\mathrm{V}<0]$, ce cas ne peut se produire que si $u_{n}-\sqrt{g} y_{0}<0$, c'est-à-dire si le régime permanent et uniforme qui existe dans le canal avant le passage de l'intumescence est fluvial. Nous nous placerons désormais dans cette hypothèse.

$\checkmark$ étant négatif, la condition précédente peul s'écrire (cas d'une onde de faible célérité) :

$$
|V|<\sqrt{g y_{0}}--u_{0}
$$

La courbe $\left(\mathrm{C}_{0}\right)$ et le profil en long de l'intumessence à un instant donné, sont représentés sur les figures 55 et 56 .

Ce phénomène peut s'observer dans un canal où est réalisé un écoulement permanent et uniforme lorsque le plan d'eau à l'extrémité aval s'élève avec une vitesse constante. Lorsque la vitesse de montée est faible, la célérité de l'onde satisfait à la condition $|\mathrm{V}|<\sqrt{g} y_{0}-u_{0}$ et on observe une « intumescence à courbure insensible » suivant l'expression employée par Boussinesq. C'est le cas ordinaire des courants de marée qui remontent l'estuaire des fleuves. La courbe $\left(\mathrm{C}_{0}\right)$ est alors voisine de $(\mathrm{H})$ el les équations (30) coincident pratiquement avec celles de SAINT-VENANT.

DEUXIÈme CAs :

$$
y_{0}<y_{0} \quad|V|>\sqrt{g y_{0}}-u_{0}
$$

Il n'en est plus de même Iorsque la célérité de

\footnotetext{
(*) Cf. la Howille Blanche, n" 3, 1953, p. 381.
}

l'onde est grande $|V|>\sqrt{g y_{0}}-u_{0}$. Ce cas se rencontre dans l'étude de la propagation de l'onde marée, dans le lit de certains fleuves, lorsque la vitesse de montée du flot est forte et donne lieu au phénomène connu sous le nom de « Mascaret $»$. Il peut s'observer également dans les canaux d'amenée des usines hydroélectriques lorsque les disjoncteurs sautent et que les régulateurs ferment rapidement l'arrivée d'eau sur les

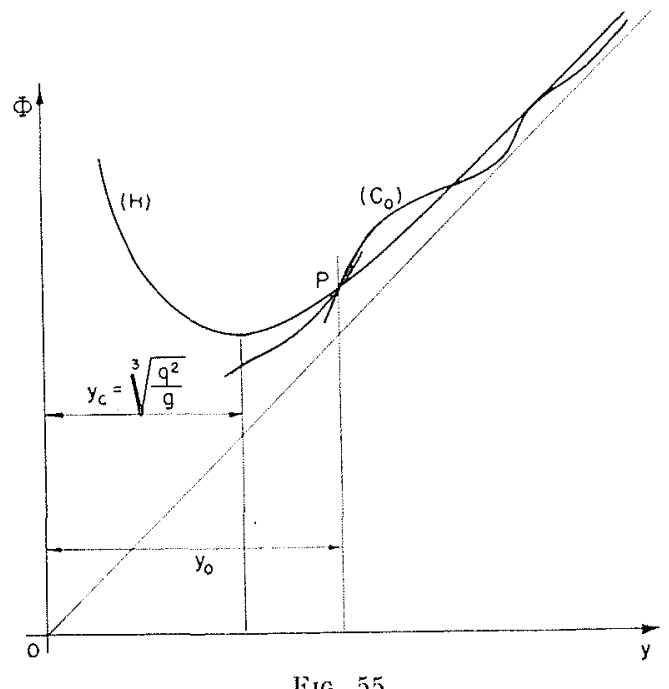

turbines. Les équations classiques de première approximation ne sont plus applicables car on ne peut plus négliger la courbure de l'onde. Il est d'ailleurs impossible d'obtenir une solution des équations de SAINT-Venant se raccordant avec le régime permanent uniforme à l'infini amont. La theorie classique conduit alors à admettre l'existence d'une discontinuité constituée par un ressaut en régime variable animé de la célérité $V$. Ce phénomène ne s'observe, en pratique,

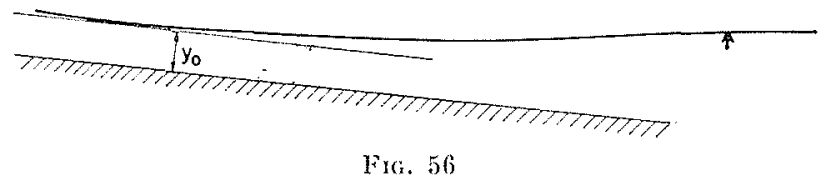

que lorsque la hauteur de l'intumescence est grande par rapport au tirant d'eau initial avant le passage de celle-ci.

Au contraire, lorsque la hauteur normale $y_{0}$ est peu inférieure à $y_{0}$, on voit apparaître comme en régime permanent un ressaut ondulé constitué par une onde initiale de faible courbure suivie de quelques ondulations. Le profil de l'onde initiale est d'ailleurs voisin de celui de l'onde solitaire classique de mềme célérité. 


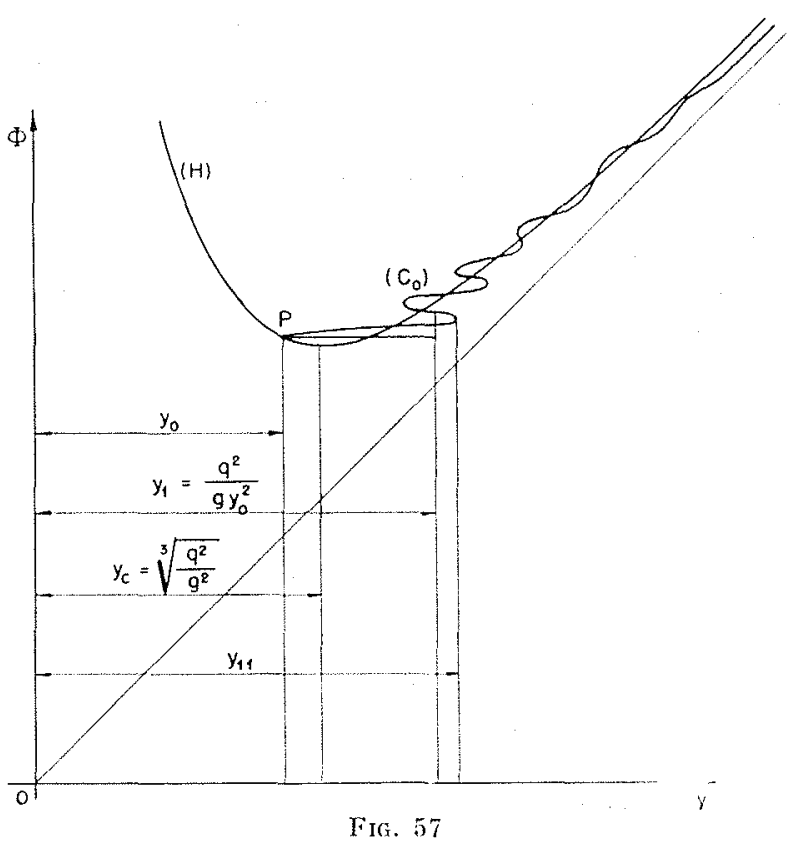

Les équations (30) de seconde approximation vont nous permettre de donner une théric du ressaut ondulé et de calculer l'amplitude et la longueur d'onde des ondulations. La courbe $\left(\mathrm{C}_{0}\right)$ et le profil en long de l'intumeseence à un instant ou en tenant compte du fait que $\mathrm{V}$ est négatif :

$$
\mathrm{V}=u_{0}-\sqrt{g y_{1}}
$$

Ainsi se trouvent confirmées les constatations expérimentales sur le profil du front de l'intumescence. Par ailleur's, $y_{1}$ étant peu inférieur a $y_{11}$ dans l'hypothèse d'un ressaut ondulé $\left(y_{0}\right.$ voisin de $y_{c}$ ), la célérité de l'intumescence est en valeur absolue légèrement inférieure à $\sqrt{g y_{11}}-u_{0}, y_{11}$ étant l'ordonnée du sommet de l'onde initiale. Ce résultat est conforme aux expériences de BAzIN sur les remous d'une certaine longueur.

En ce qui concerne l'amplitude et la longueur d'onde des ondulations qui suivent l'onde initiale, il est possible de les déterminer par une intégration aux différences finies des équations (30). On peut également, comme dans l'étude des nappes ondulées, remplacer la courbe $\left(\mathrm{C}_{0}\right)$ par unc ligne en escalier.

Cela revient à confondre le front de l'intumescence avec l'onde solitaire (S). L'ordonnéc du sommet de l'onde initiale est ainsi :

$$
y_{11}=y_{1}==\frac{q^{2}}{g y_{0}^{2}}=\frac{\left(u_{01}-V\right)^{2}}{g}
$$

tenant compte de l'équation de l'onde solitaire,

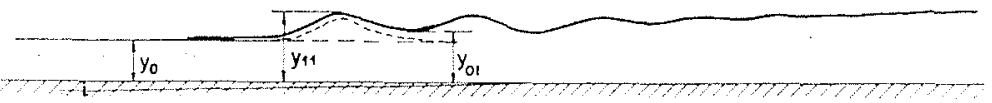

Fic. 58

donné sont représentés respectivement sur les figures 57 et 58 .

En se reportant à l'étude du ressaut ondulé en régime permanent, en voit que l'onde initiale est située au-dessus de la courbe intégrale (S) de l'équation différentielle (17):

$$
\frac{q^{2}}{3 g}\left(\frac{d y}{d x}\right)^{2}=\left(y-y_{0}\right)^{2}\left[\frac{q^{2}}{g y_{0}^{2}}-y\right]
$$

Cette courbe (S), représentée en pointillé sur la figure 58, est très voisine, dans sa partie amont, du front de l'intumescence; l'ordonnéc de son sommet $y_{1}=\left(q^{2} / g y_{0}{ }^{2}\right)$ diffère peu de l'ordonnée $y_{11}$ du sommet de l'onde initiale. Or, la courbe (S) est précisément l'onde solitaire dont la vitesse de propagation dans le courant de vitesse $u_{0}$ est égale à la célérité de l'intumescence.

On a en effet :

$$
g y_{1}=\frac{q^{2}}{y_{0}^{2}}=\left(u_{0}-\mathrm{V}\right)^{2}
$$

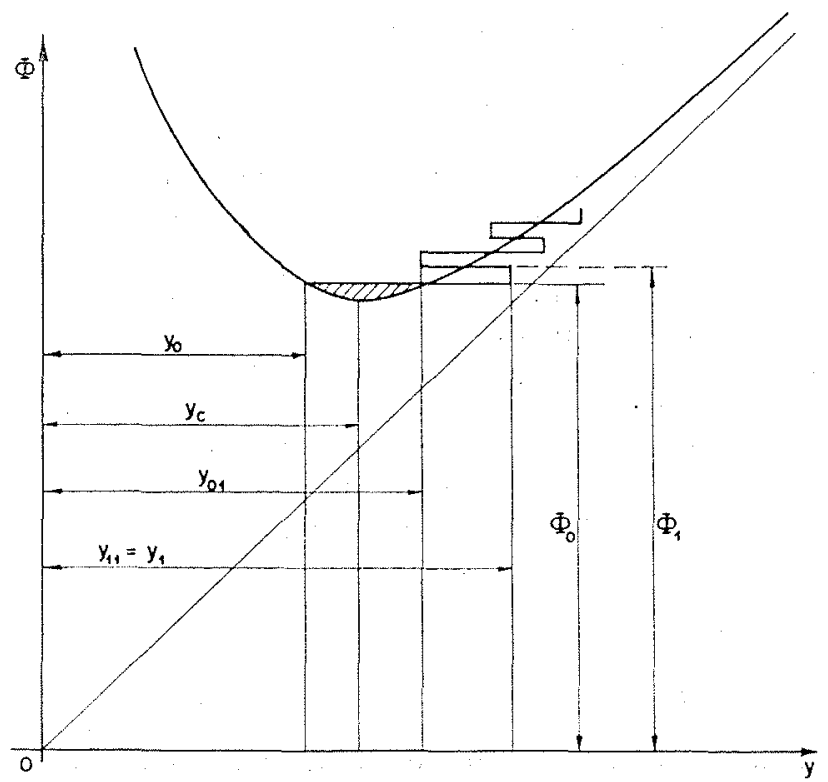

FIG. 59 
on calcule la variation de $\Phi$ à partir de $\Phi_{0}=y_{0}+q^{2} /\left(2 g y_{0}{ }^{2}\right)$ lorsque $y$ croît de $y_{0}$ à $y_{1}$ en utilisant la relation :

$$
\frac{d \Phi}{d x}=i-\frac{u^{2}}{y c^{2}(y)}
$$

soit $\Phi_{1}$ la valeur de $\Phi$ pour $y=y_{1}$ ainsi calculée, l'ordonnée $y_{01}$ du point bas de la première ondulation s'obtiendra à partir de la relation déjà démontrée :

$$
\Phi_{1}=\frac{y_{1}+y_{01}}{2}+\frac{2}{2 g y_{1} y_{01}}
$$

équation dont il convient de prendre la racine la plus grande. La demi-longueur d'onde $\mathrm{L}_{0}$, distance entre les deux premiers sommets de l'intu- mescence, sera alors donnée par l'intégrale ellip. tique :

$$
\mathrm{L}_{0}=\frac{\eta \int_{-1}^{+1} \frac{d u}{\sqrt{\left(1-u^{2}\right)(1+K u)}}}{\sqrt{3 g\left[\frac{y_{01}+y_{1}}{2}-\frac{q^{2}}{g y_{01} y_{1}}\right]}}
$$

avec :

$$
\mathbf{K}=\frac{y_{1}-y_{01}}{y_{1}+y_{01}-\frac{2 q^{2}}{g y_{01} y_{1}}}
$$

On déterminerait ainsi de proche en proche l'amplitude et la longueur d'onde des ondulations. La courbe $\left(\mathrm{C}_{0}\right)$ étant asymptote à $(\mathrm{H})$, cette amplitude s'amortit rapidement et le profil de l'intumescence tend asymptotiquement vers une droite horizontale.

\section{INTUMESCENCES A CÉLERITÉ CONSTANTE SE RACCORDANT A L'INFINI AVAL AVEC LE RÉGLE PERMANENT UNIFORME.}

Ce sont les solutions du systeme (30) asymptotiques à la solution particulière $y=y_{0} \Phi=\omega_{0}$ lorsque $x$ augmente indéfiniment par valeurs positives. Elles correspondent aux deux racines non positives $m_{2}$ et $m_{3}$ de l'équation caractéristique $\left(13_{a}\right)$. Ces racines sont, en général, imaginaires conjuguées sauf lorsque la célérité de propagation est en valeur absolue largement supérieure à $\sqrt{g y_{0}}-u_{0}$, mais leur partie réelle est négative. L'étude de ces solutions est identique à celle précédemment faite à propos du régime permanent. Il en existe une double infinité dépendant de deux constantes arbitraires.

De telles intumescences peuvent être obtenues de la manière suivante. Considérons un canal rectangulaire de grande largeur, de pente $i$, dans lequel est réalisé un ćcoulement permanent uniforme. Désignons par $y_{0}$ la hauteur d'eau normale et $u_{0}$ la vitesse du courant. Nous supposerons également le régime fluvial $u_{0}<\sqrt{g} y_{0}$.

Considérons alors une vanne d'ouverture don- née $a$, barrant toute la largeur du canal et se déplaçant dans ce dernier avec une vitesse constante $V$ (on suppose $V<0$, la vanne se déplace vers l'amont).

Au bout d'un temps très court, un etat de régime s'établit. On crée de cette manière à l'amont de la vanne une intumescence $\left(\mathrm{I}_{1}\right)$ de célérité $V$ se raccordant à l'amont avec le régime uniforme existant auparavant et qui est analogue à celles étudiées au paragraphe précédent. Sous certaines conditions, on voit également apparaître, à l'aval de la vanne, une intumescence $\left(I_{2}\right)$ de célérité $V$ se raccordant à l'aval avec le régime uniforme de vitesse $u_{0}$.

On est évidemment conduit à distinguer deux cas suivant que le régime est fluvial ou torrentiel par rapport à des axes animés de la vitesse $Y$ de déplacement de la vanne. La forme des intumescences correspondant à ces deux cas est représentée sur les figures 60 et 61 .

$$
1^{\circ} \quad|V|<\sqrt{g y_{0}-u_{0}}
$$

a) Cas d'Une grande ouverture a DE la vanne.

Le ressaut est noyé à l'aval de celle-ci. L'intumescence $\left(\mathrm{I}_{2}\right)$ n'existe pas $(\mathrm{fig} .60 \mathrm{r}$ ) .
B) Cas d'Une ouverture MOYfnne dE LA VANne.

Ressaut ondulé à l'aval de la vanne (fig. $60 \beta$ ).

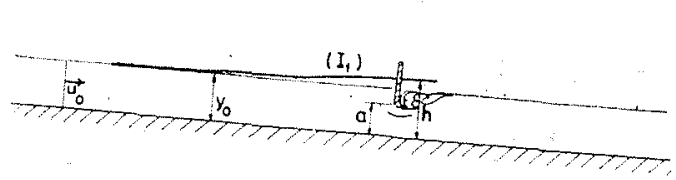

F1G. $60 \%$

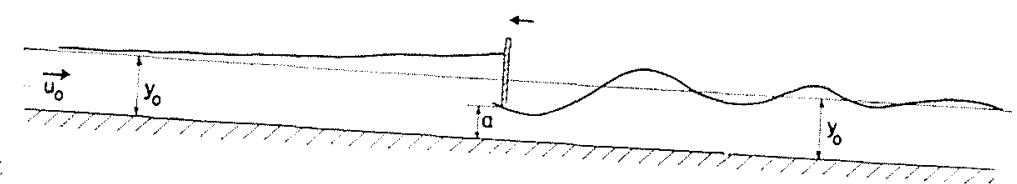

FIG. $60 \beta$ 
y) Cas d'unl ouverture famle de la vanne. - Ressaut déferlant à l'aval (fig. $60 \%$ ).

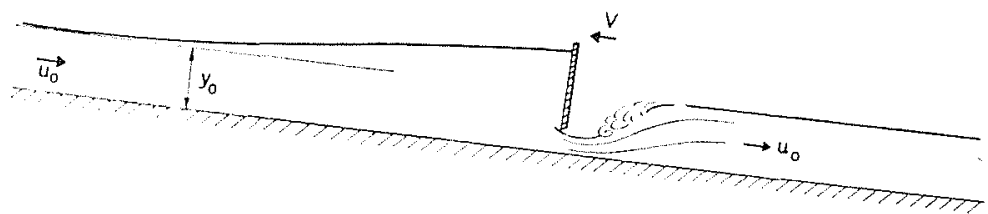

FIG, $60 \gamma$

$$
\text { 2" } \quad \mathrm{V}>\mathrm{V} g y_{0}-u_{0}
$$

Dans tous les cas, le problème à résoudre est le suivant : Pour une ouverture $a$ et une vitesse de translation $V$ de la vanne, déterminer la hauteur d'eau $h$ à l'amont de la vanne et les courbes $\left(I_{1}\right)$ et $\left(I_{2}\right)$.

Nous utiliserons des axes $O x$ et Oy représentés sur la figure 61 et animés de la vitesse $V$ de translation de la vanne.

Par rapport à ces axes, les intumescences $\left(I_{1}\right)$ et $\left(\mathrm{I}_{2}\right)$ d'équations $y=y_{1}(x) \quad y=y_{2}(x)$ sont des solutions du systeme:

$$
\left\{\begin{array}{l}
\frac{q^{2}}{6 g}\left(\frac{d y}{d x}\right)^{2}=\Phi y^{2}-\frac{y^{3}}{2}+\frac{q^{2}}{2 g}-\mathrm{F} y \\
\frac{d \Phi}{d x}=i \cdots \frac{u^{2}}{y c^{2}(y)} \\
\frac{d \mathrm{~F}}{d x}=i y-\frac{u^{2}}{c^{2}(y)} \\
u=\mathrm{V}+-\frac{q}{y}
\end{array}\right.
$$

qui, on le démontre aisément, est équivalent aux deux systèmes (26) et (30).

$q$ est une constante ayant pour valeur :

$$
q=\left(u_{0}-V\right) y_{0}
$$

Il faut rechercher deux solutions :

$$
\begin{array}{llll}
\left(\mathrm{S}_{1}\right) & y=y_{1}(x) & \Phi=\Phi_{1}(x) & \mathrm{F}=\mathrm{F}_{1}(x) \\
\left(\mathrm{S}_{2}\right) & y=y_{2}(x) & \Phi=\Phi_{2}(x) & \mathrm{F}=\mathrm{F}_{2}(x)
\end{array}
$$

du système (31) asymptotiques respectivement pour $x=-\infty$ et $x=+\infty$ à la solution particulière:

$$
\begin{aligned}
& y=y_{0} \quad \Phi=\Phi_{0}=y_{0}+\frac{q^{2}}{2 g y_{0}{ }^{2}} \\
& \mathrm{~F}==\mathrm{F}_{0}=\frac{y_{0}^{2}}{2}+\frac{q^{2}}{g y_{0}}
\end{aligned}
$$

$\left(S_{1}\right)$ et $\left(S_{2}\right)$ dépendent donc respectivement d'une et de deux constantes arbitraires. Ces conslantes seront déterminées par les conditions aux limites au droit de la vanne $[x=0]$.

Une première condition évidente s'écrit :

$$
y_{2}(0)=a
$$

a étant l'ouverture de la vanne.

Les deux autres s'obtiennent en appliquant lo théorème de Berroullr et le théorème des quantités de mouvement à la portion de fluide comprise entre la section de la vanne et une section située légèrement en amont, ces deux sections étant animées de la vitesse de translation $V$.

Un calcul immédiat, déjà effectué lors de l'étude du régime permanent, conduit aux deux relations :

$$
\begin{gathered}
\Phi_{1}(0)=\Phi_{2}(0) \\
\mathrm{F}_{1}(0)=\mathrm{F}_{2}(0)+\frac{\mathrm{P}}{\circ g}
\end{gathered}
$$

oì $P$ représente la poussée du fluide sur la face amont de la vanne.

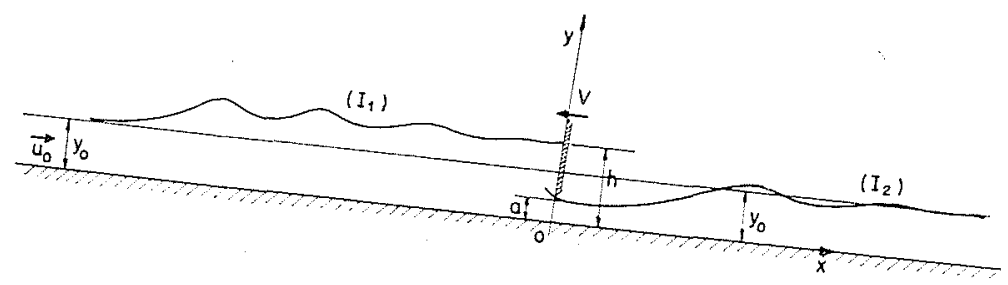

FIG. 61 


\section{Etude du ressaut déferlant en régime variable.}

Nous terminerons ces considérations sur les intumescences à célérité constante par une théorie élémentaire du ressaut qui nous permettra de retrouver les résultats obtenus par BARRÉDE-Sant-VEnaNT, tout en précisant leurs limites d'application.

Les développements précédents sur le ressaut ondulé ne sont valables que lorsque la hauteur. du corps de l'intumescence est inférieure au tirant d'eau initial. Lorsque cette hauteur augmente, on observe un raidissement du front de londe initiale, qui finit par déferler. Les équations (26) qui supposent la continuité de la ligne d'eau ne sont plus applicables; on observe d'ailleurs, mème avant le déferlement, une variation de la répartition des vitesses entre deux sections voisines et nos équations ne peuvenl plus alors que traduire l'allure du phénomène, c'est-à-dire la tendance au raidissement et au diferlement du front de l'intumescence.

En fait, ce déferlement apparaît, pour unc hauteur du corps de l'intumescence bien inférieure à la hauteur théorique qu'on pourrait déduire de nos équations. [Voir théorie du déferlement du ressaut en régime permanent.]

L'onde initiale perd sa forme caractéristique el les ondulations qui la suivent finissent par disparaître entièrement. Celte discontinuité dans l'écoulement s'accompagne nécessairement d'une perte d'énergie localisée qui correspond à une variation de la fonction qui intervient dans les équations (30) par application du théorème de BERnoull généralisé. On peut par contre admettre qu'entre deux sections situées à l'amont et à l'aval du ressaut il n'y a pas discontinuité de la fonction $\mathrm{F}$ qui intervient dans l'application du théorème des quantités de mouvement à condition de négliger entre ces deux sections l'influence des forces de pesanteur et de frottement, hypothèse qui est valable dans le cas d'un ressaut déferlant. Comme on peut également dans la section aval négliger la courbure et la pente de la ligne d'eau par suite de la disparition des ondulations, on est conduit, par application des équations (26) et eu égard à la discontinuité de la surface libre, aux deux relations suivantes :

$$
\left\{\begin{array}{l}
\mathrm{F}\left(y_{0}\right)=\frac{y_{0}^{2}}{2}+\frac{q^{2}}{g y_{0}}=\frac{y_{1}^{2}}{2}+\frac{q^{2}}{g y_{1}}=\mathrm{F}\left(y_{1}\right) \\
\left(u_{0}-\mathrm{V}\right) y_{0}=\left(u_{1}-\mathrm{V}\right) y_{1}=q
\end{array}\right.
$$

$y_{0}$ et $y_{1}$ désignant respectivement les deux sections situées à l'amont et à l'aval du ressaut. Les équalions (30) donnent alors la discontinuité de la fonction ir au pasasge du ressaut, c'est-à-dire la perte d'énergie localisée :

$$
\Phi\left(y_{1}\right)-\Phi\left(y_{0}\right)=y_{1}+\frac{q^{2}}{2 g y_{1}{ }^{2}}-y_{0}-\frac{q^{2}}{2 g y_{0}{ }^{2}}
$$

Considérons dans ces conditions un canal rectangulaire de grande largeur où est réalisé un écoulement permanent uniforme de vitesse $u_{0}$ correspondant à une hauteur normale $y_{0}$. Le débit dans une section quelconque est $\mathrm{Q}_{0}=u_{0} y_{0}$. Supposons alors qu'on maintienne à l'amont d'une section déterminée du canal un débit constant $Q_{1}<Q_{0}$ par un réglage convenable d'une

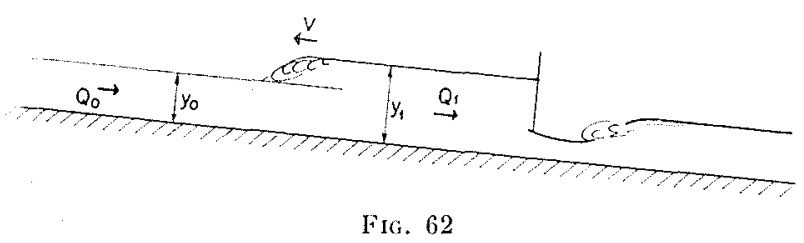

vanne placée dans cette section. On crée dans ces conditions à l'amont de la vanne une intumescence qui remonte le courant avec une certaine célérité $V$.

On se propose de déterminer, dans l'hypothèse d'un ressaut déferlant la vitesse de propagation $V$ de l'intumescence et sa hauteur $y_{1}$.

Les relations (32) s'écivent dans ces conditions:

$$
\begin{gathered}
\frac{y_{0}^{2}}{2}+\frac{q^{2}}{g y_{0}{ }^{2}}=\frac{y_{1}^{2}}{2}+\frac{q^{2}}{g y_{1}^{2}} \\
\left(u_{0}-\mathrm{V}\right) y_{0}=\mathrm{Q}_{0}-\mathrm{V} y_{0}=\left(u_{1}-\mathrm{V}\right) y_{1} \\
\quad=\mathrm{Q}_{1}-\mathrm{V} y_{1}=q
\end{gathered}
$$

d'où on tire respectivement :

$\left(\mathrm{C}_{1}\right) \quad q^{2}=g \frac{y_{1} y_{0}}{2}\left(y_{1}+y_{0}\right)$

$$
q=\frac{\mathrm{Q}_{0} y_{1}-\mathrm{Q}_{1} y_{0}}{y_{1}-y_{0}}
$$

Les courbes représentatives de ces deux fonctions de $y_{1}$ sont tracées sur les figure $\left(63_{1}\right)$ et $\left(63_{2}\right)$. 


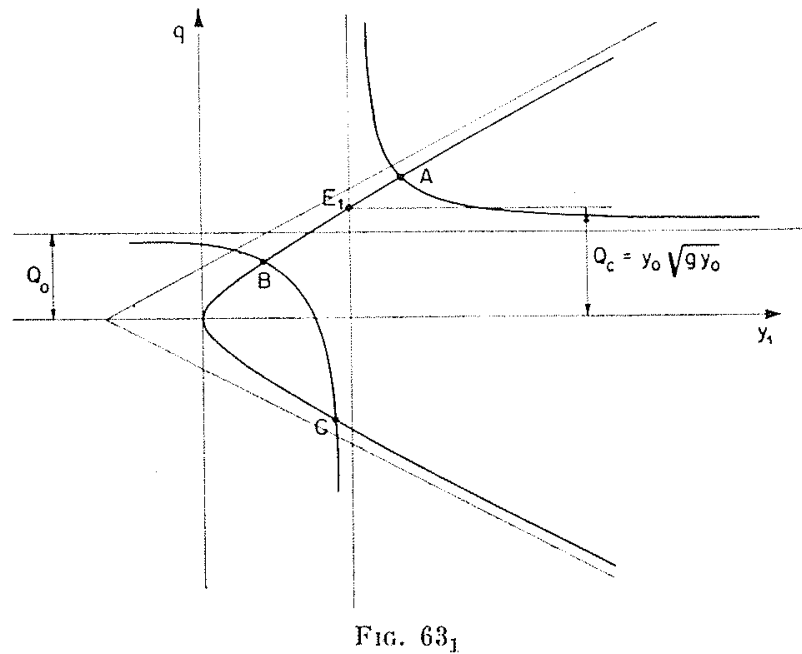

Les deux cas de figures $\left(63_{1}\right)$ et $\left(63_{2}\right)$ correspondent aux deux hypothèses :

(63. $3_{1} \quad Q_{0}<y_{0} \sqrt{g y_{0}}$, régime permanent fluvial dans le canal,

$\left(63_{2}\right) \quad Q_{0}>y_{0} \sqrt{g y_{0}}$, régime permanent torrentiel dans le canal.

La hauteur $y_{1}$ de l'intumescence est donnée par l'abscisse des points d'intersection des courbes $\left(\mathrm{C}_{1}\right)$ et $\left(\mathrm{C}_{2}\right)$. Le problème admet en général trois solutions dont l'une au moins correspondant au point A est réelle.

Les deux solutions qui correspondent aux points $B$ et $C$ donnent des hauteurs d'intumescences $y_{1}$ inférieures à $y_{0}$.

Elles concernent des ondes descendantes à célérité positive $V$ dont l'une est plus rapide et l'autre moins rapide que le courant de vitesse $u_{0}$.

La solution correspondant à une onde remontant le courant $[\mathrm{V}<0]$ est donnée par l'abscisse $y_{1}$ du point $A$.

Sa vitesse de propagation :

$$
V=u_{0}-\frac{q}{y_{0}}=u_{0}-\sqrt{g y_{1} \times \frac{1}{2}\left(1+\frac{y_{1}}{y_{0}}\right)}
$$

sa célérité relative par rapport au courant de vitesse $u_{0}$ :

$$
\mathrm{V}_{r}=-\sqrt{g y_{1} \times \frac{1}{2}\left(1+\frac{y_{1}}{y_{0}}\right)}
$$

constitue la formule classique de BARRÉ-DFSAINT-VENANT.

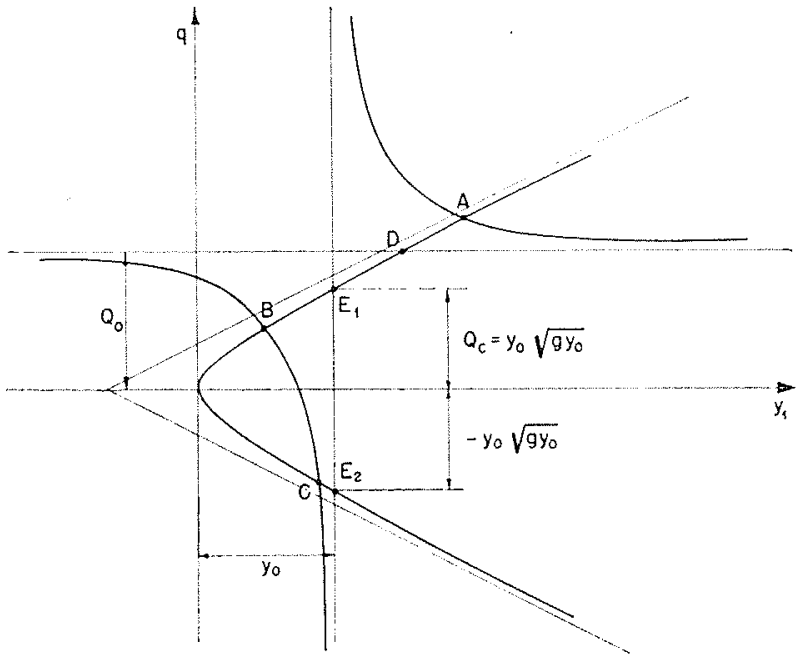

Fic, 632

Cherchons maintenant la limite de la hauteur $y_{1}$ de l'intumescence lorsque le débit $Q_{1}$ à l'aval du ressaut tend vers $\mathrm{Q}_{0}$. Cette limite, on le voit immédiatement sur les figures $\left(63_{1}\right)$ et $\left(63_{2}\right)$, dépend de la nature du régime permanent uniforme réalisé dans le canal avant le passage de l'onde.

1 "Régime fluviat : $Q_{0}<y_{0} \sqrt{g y_{0}}$. Lorsque $Q_{1}$ tend vers $Q_{0}$, le point $A$ de $\left(63_{1}\right)$ tend vers le point $\mathrm{E}_{1}$ d'abscisse $y_{0} ; y_{1}$ tend vers $y_{0}$, l'amplitude de l'intumescence tend vers 0 . Il ne peut donc exister d'intumescence stationnaire en régime fluvial permanent $\left[Q_{1}=Q_{0}\right]$.

$2^{\circ}$ RÉGIME TORRENTIEL : $\mathrm{Q}_{0}>y_{0} \sqrt{g y_{0}}$. Lorsque $Q_{1}$ tend vers $Q_{0}$, le point $A$ de $\left(63_{2}\right)$ tend vers le point $D$ d'intersection de $\left(C_{1}\right)$ avec la droite $q=Q_{0}$. La hauteur de l'intumescence $y_{1}$ tend donc vers une limite $y_{1 p}$ différente de $y_{0}$ qui constitue la racine positive de l'équation :

$$
\frac{2 \mathrm{Q}_{0}^{2}}{g}=y_{1 p} y_{0}\left(y_{1 p}+y_{0}\right)
$$

Cette relation est précisément l'équation caractéristique du ressaut en régime permanent.

La vitesse de propagation de l'intumescence est donnée par :

$$
V=u_{0}-\frac{q}{y_{0}}=\frac{Q_{0}-q}{y_{0}}=0
$$

Elle est alors nulle.

Ainsi se trouve justifié le fait de considérer comme une onde stationnaire le ressaut en régime permanent. 